\title{
Development of Computational and Experimental Benchmark Data for Wind Turbine Aeroelastic Algorithms
}

\author{
by \\ Mohamed El Sherif, B.Eng. \\ A thesis submitted to the \\ Faculty of Graduate and Postdoctoral Affairs \\ in partial fulfillment of the requirements for the degree of
}

\author{
Master of Applied Science in Mechanical Engineering \\ Ottawa-Carleton Institute for Mechanical and Aerospace Engineering \\ Department of Mechanical and Aerospace Engineering \\ Carleton University \\ Ottawa, Ontario \\ April, 2014 \\ (C) Copyright
}

Mohamed El Sherif, 2014 


\section{Abstract}

Efficient wind turbines with high power to cost ratio require rotor blades of high quality. This requires a comprehensive ability to predict a blade's aerodynamic performance and structural response when exposed to a variety of air flow conditions. Aeroelastic algorithms that are able to achieve this efficiently and rapidly as a tool for preliminary design stages are an ongoing concern for the professional practitioners. These aeroelastic algorithms require validation data upon which their accuracy can be determined.

To this end, a validation process was investigated and applied to determine the accuracy of the Preliminary Aeroelastic Analysis of Wind Turbine (PAAC-WTB) algorithm, an algorithm that was recently developed in the Advanced Dynamic Research gtoup. A preliminary wind tunnel test was performed on a 3D-printed reduced scale model of the National Renewable Energy Laboratory (NREL) S809 blade, and experimental results were obtained to compare against algorithm results. The results show some agreement, providing a basis for further experimental validation to be undertaken. Moreover, CFD and FEA analysis was then performed, using ANSYS, on the S809 airfoil and the S809 experimental blade for various conditions and parameter values. Comparison of algorithm results to ANSYS results showed good agreement. Thus, the research concluded that the validation process was a success. 


\section{Acknowledgments}

I would like to thank and extend my sincere appreciation for my thesis supervisors, Professors Fred F. Afagh and Robert G. Langlois for their support and patience throughout the project. Their wisdom on all matters pertaining to this project was invaluable and I was fortunate to have them as my supervisors. Thanks and appreciation as well to my colleague and friend Alex McFarlane, a very talented and hard working individual and the main component for this project. May you always traverse difficult elements with fluidity.

Major thanks and appreciation as well to two great guys, Carleton Laboratory Technologists Steve Truttman (aka the electronics wizard) and David Raude for their assistance throughout the wind tunnel aspect of this project. Both are truly assets to Carleton.

Thanks and gratitude as well go to my family who supported me for the last two

years. My father for convincing me to go ahead and do my Masters program and my mother for her wisdom on matters of life. May I always be blessed with their guidance. 


\section{Table of Contents}

1 Introduction 1

1.1 Motivation . . . . . . . . . . . . . . . . . . . 1

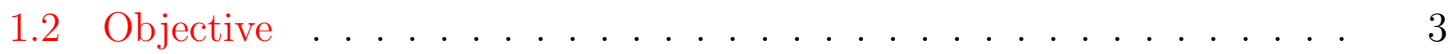

1.3 Overview . . . . . . . . . . . . . . . . . . . 3

2 Literature Review 5

2.1 Rotor Blade Aerodynamics . . . . . . . . . . . . . . . . . 5

2.2 Actuator Disc Theory . . . . . . . . . . . . . . . . 8

2.3 Blade Element Momentum Theory . . . . . . . . . . . . . . . . 12

2.4 Blade Design Optimization . . . . . . . . . . . . . . . 17

2.5 Betz's Method . . . . . . . . . . . . . . . 17

2.6 Schmitz's Method . . . . . . . . . . . . . . . . . 20

3 Blade Design and Wind Tunnel Experiment 24

3.1 Rotor Blade Design . . . . . . . . . . . . . . 25

3.1.1 Single Blade Design and Manufacturing . . . . . . . . . . . . 25

3.1.2 HAWT Blade Design . . . . . . . . . . . . . . . . 33

3.2 Wind Tunnel Experiment . . . . . . . . . . . . . . . . 38

3.2.1 Wind Tunnel Experiment Results . . . . . . . . . . . . . . . . 41 
4 CFD and FEA Analysis $\quad 46$

4.1 S809 Two-dimensional CFD Analysis . . . . . . . . . . . . . . . . 46

4.1.1 Static 2D CFD Analysis . . . . . . . . . . . . . . 47

4.1.2 Dynamic 2D CFD analysis . . . . . . . . . . . . . 53

4.1.3 Theodorsen's Solution for Harmonic Airfoil Motion . . . . . . 58

4.1.3.1 Pitching Motion . . . . . . . . . . . . 59

4.1.3.2 Pitching and Plunging Motion . . . . . . . . . . . 62

4.1.4 Wagner's Solution Airfoil Motion . . . . . . . . . . . . . 65

4.2 3D CFD and FEA Analysis . . . . . . . . . . . . . 67

4.2.1 Modal Response and Forced Response Analysis . . . . . . . . 68

4.2.1.1 Analysis of Aluminum Tube . . . . . . . . 68

4.2.1.2 Analysis of the S809 Blade . . . . . . . . . . . 71

4.2.2 3D Aeroelastic Analysis . . . . . . . . . . . . . . . . . 74

4.3 Mesh Convergence . . . . . . . . . . . . . . . . . 84

5 Conclusion $\quad 86$

5.1 Future Work and Recommendations . . . . . . . . . . . . . 87

5.2 Scientific Contribution . . . . . . . . . . . . . . 87

$\begin{array}{lr}\text { References } & 88\end{array}$

$\begin{array}{ll}\text { Appendix A } & 91\end{array}$

A.1 Fluent User Defined Functions . . . . . . . . . . . . . . . . . . . 91

$\begin{array}{ll}\text { Appendix B } & 93\end{array}$ 


\section{List of Tables}

3.1 PA2200 material properties . . . . . . . . . . . . . . . 30

3.2 Description of blade designs. . . . . . . . . . . . . . . . . 33

3.3 Wind tunnel test trials. . . . . . . . . . . . . . . . . . . . . . 39

3.4 Wind tunnel experiment data acquisition instrumentation. . . . . . 41

4.1 2D simulation parameters. . . . . . . . . . . . . . . 51

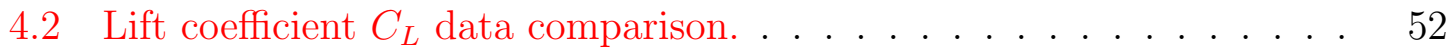

4.3 CFD simulation parameters for impulse functions. . . . . . . 56

4.4 CFD simulation parameters for pitching-only motion. . . . . . . . 60

4.5 CFD simulation parameters for pitching and plunging motion. . . . . 63

4.6 CFD simulation parameters for Wagner's motion. . . . . . . . . 65

4.7 Aluminum tube material properties and dimensions. . . . . . . 68

4.8 Aluminum tube modal response natural frequencies. . . . . . . . . . 69

4.9 S809 blade model material properties and dimensions. . . . . . . . . 72

4.10 S809 modal response natural frequencies. . . . . . . . . . . . . 72

4.11 Simulation parameters for aeroelastic trials. . . . . . . . . . 81

4.12 Simulation parameters for mesh convergence comparison. . . . . . . . 84

4.13 Lift coefficient mesh convergence results. . . . . . . . . . . 85 


\section{List of Figures}

2.1 Rotor airfoil rotating in freestream flow . . . . . . . . . . . . 5

2.2 Turbine flow control volume. . . . . . . . . . . . . . . . 8

2.3 Speeds in the rotorplane defined upstream, at the rotor plane and downstream. . . . . . . . . . . . . . . . . . . 14

2.4 Flow over rotor blade. . . . . . . . . . . . . . . . . . . . 16

2.5 Rotor blade element. . . . . . . . . . . . . . . . . . . . 18

3.1 S809 airfoil. . . . . . . . . . . . . . . . 26

3.2 S833 airfoil. . . . . . . . . . . . . . . . 26

3.3 S834 airfoil. . . . . . . . . . . . . . . . . . . 26

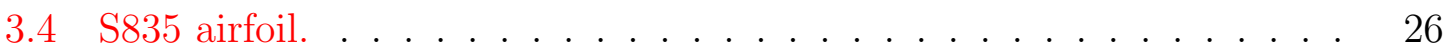

3.5 S809 blade shaded model. . . . . . . . . . . . . . . . . 26

3.6 Blade with removable section. . . . . . . . . . . . . 26

3.7 Pressure distribution and structural deformation of S809 blade at $15 \frac{\mathrm{m}}{\mathrm{s}} .27$

3.8 Load cell interface. . . . . . . . . . . . . . . . . . . . 28

3.9 Clamshell mould. . . . . . . . . . . . . . . . . . . . . . . . 29

3.10 Blade deflection for $V_{0}=55 \frac{\mathrm{m}}{\mathrm{s}} \ldots \ldots \ldots \ldots . \ldots . \ldots 29$

3.11 Safety factor for $V_{0}=55 \frac{\mathrm{m}}{\mathrm{s}} \ldots \ldots \ldots . \ldots \ldots$

3.12 Printed models with defects. . . . . . . . . . . . . . . . 31

3.13 Alignment jigs. . . . . . . . . . . . . . . . . . . 31 
3.14 Assembled blade. . . . . . . . . . . . . . . . . . . . . . 32

3.15 Hybrid Schmitz blade. . . . . . . . . . . . . . . . . . . 34

3.16 Hybrid linear blade. . . . . . . . . . . . . . . . . . . . . 34

3.17 Power coefficient versus tip speed ratio. . . . . . . . . . . . 35

3.18 Thrust coefficient versus tip speed ratio. . . . . . . . . . 36

3.19 Preliminary HAWT design model. . . . . . . . . . . . . . . . . . 37

3.20 S809 blade installed in the test section. . . . . . . . . . . . 38

3.21 Test section in position in the closed loop wind tunnel. . . . . . . . . 39

3.22 Data acquisition instrumentation. . . . . . . . . . . . . 40

3.23 Filtered load cell data. . . . . . . . . . . . . . . . . . . 42

3.24 Lift results at -10 deg angle of attack and $V_{0}=17.3 \frac{\mathrm{m}}{\mathrm{s}} \ldots \ldots \ldots 43$

3.25 Lift results at -10 deg angle of attack and $V_{0}=19.7 \frac{\mathrm{m}}{\mathrm{s}} \ldots \ldots \ldots 43$

3.26 Lift results at 0 deg angle of attack and $V_{0}=17.3 \frac{\mathrm{m}}{\mathrm{s}} \ldots \ldots \ldots 44$

3.27 Lift results at 0 deg angle of attack and $V_{0}=19.7 \frac{\mathrm{m}}{\mathrm{s}} \ldots \ldots \ldots 44$

3.28 Lift results at $10 \mathrm{deg}$ angle of attack and $V_{0}=17.3 \frac{\mathrm{m}}{\mathrm{s}} \ldots \ldots \ldots 45$

3.29 Lift results at $10 \mathrm{deg}$ angle of attack and $V_{0}=19.7 \frac{\mathrm{m}}{\mathrm{s}} \ldots \ldots \ldots .45$

4.1 S809 airfoil square grid unstructured mesh. . . . . . . . . . . 47

4.2 Unstructured refined mesh around S809 airfoil. . . . . . . . . . . . 48

4.3 Unstructured mesh computational domain. . . . . . . . . . . . . 48

4.4 S809 airfoil C-grid structured mesh. . . . . . . . . . . . . . . 49

4.5 Structured refined mesh around the S809 airfoil. . . . . . . . . . . 49

4.6 Structured mesh computational domain. . . . . . . . . . . . 50

4.7 Lift coefficient data comparison . . . . . . . . . . . . 53

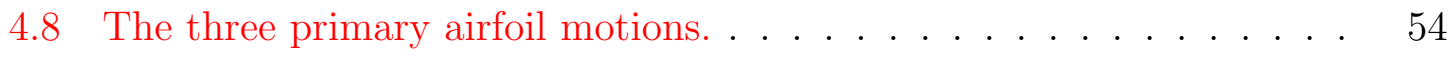

4.9 Airfoil motion variables . . . . . . . . . . . . . . . . . . 55

4.10 Plot of non-harmonic motion functions $\ldots \ldots \ldots \ldots$ 
4.11 Pitching only lift coefficient results. . . . . . . . . . . 57

4.12 Plunging only lift coefficient results. . . . . . . . . . . . 57

4.13 Pitching and plunging lift coefficient results. . . . . . . . . 58

4.14 2D airfoil geometry . . . . . . . . . . . . . . . . . 59

4.15 Comparison of lift coefficient data for pitching-only case, $k=0.20$, $\omega=12 \frac{\mathrm{rad}}{\mathrm{s}}$, and $V_{0}=30 \frac{\mathrm{m}}{\mathrm{s}} \ldots \ldots \ldots \ldots \ldots \ldots \ldots$

4.16 Comparison of lift coefficient data for pitching-only case, $k=0.50$, $\omega=12 \frac{\mathrm{rad}}{\mathrm{s}}$, and $V_{0}=12 \frac{\mathrm{m}}{\mathrm{s}} \ldots \ldots \ldots \ldots \ldots \ldots$

4.17 Comparison of lift coefficient data for pitching-only case, $k=1.0$, $\omega=6 \frac{\mathrm{rad}}{\mathrm{s}}$, and $V_{0}=6 \frac{\mathrm{m}}{\mathrm{s}} \ldots \ldots \ldots \ldots \ldots \ldots$

4.18 Comparison of lift coefficient data pitching and plunging, $k=0.20$, $\omega_{\text {pitch }}=12 \frac{\mathrm{rad}}{\mathrm{s}}$, and $\omega_{\text {plunge }}=12 \frac{\mathrm{rad}}{\mathrm{s}} \ldots \ldots \ldots \ldots$

4.19 Comparison of lift coefficient data pitching and plunging, $k=0.50$, $\omega_{\text {pitch }}=12 \frac{\mathrm{rad}}{\mathrm{s}}$, and $\omega_{\text {plunge }}=30 \frac{\mathrm{rad}}{\mathrm{s}} \ldots \ldots \ldots \ldots$

4.20 Comparison of lift coefficient data pitching and plunging, $k=1.0$, $\omega_{\text {pitch }}=30 \frac{\mathrm{rad}}{\mathrm{s}}$, and $\omega_{\text {plunge }}=12 \frac{\mathrm{rad}}{\mathrm{s}} \ldots \ldots \ldots \ldots$

4.21 Comparison of Wagner's function lift coefficient results, $\triangle \theta=8 \mathrm{deg}$ and $V_{0}=50 \frac{\mathrm{m}}{\mathrm{s}} \ldots \ldots \ldots \ldots \ldots \ldots \ldots \ldots \ldots \ldots \ldots \ldots \ldots \ldots$

4.22 Comparison of Wagner's function lift coefficient results, $\triangle \theta=5 \mathrm{deg}$

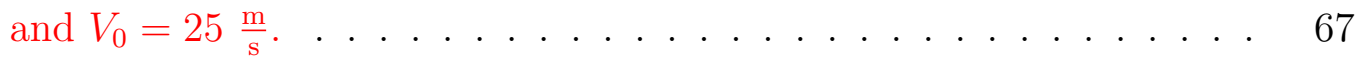

4.23 Aluminum tube with applied tip load. . . . . . . . . . . . . . 69

4.24 Aluminum tube tip deflection at $f=100 \mathrm{~Hz} \ldots \ldots \ldots \ldots \ldots$

4.25 Aluminum tube tip deflection at $f=250 \mathrm{~Hz} \ldots \ldots \ldots \ldots$

4.26 Aluminum tube tip deflection at $f=500 \mathrm{~Hz} \ldots \ldots \ldots \ldots . \ldots 71$

4.27 S809 blade with applied distributed pressure load. . . . . . . . . . 72

4.28 S809 tip deflection at $f=0.6 f_{n, 1} \ldots \ldots \ldots \ldots \ldots \ldots \ldots$ 
4.29 S809 tip deflection at $f=1.2 f_{n, 1} \ldots \ldots \ldots$. . . . . . . 74

4.30 Two way fluid/structure ANSYS Workbench setup. . . . . . . . . . . 75

4.31 S809 blade fluid solid interface in TSM and FM. . . . . . . . . . . 75

4.32 Force and displacement data transfer between the TSM and the FM. $\quad 76$

4.33 Mesh enclosures around S809 blade. . . . . . . . . . . . . . . 77

4.34 First mesh enclosure around the S809 blade. . . . . . . . . . . . . . . 77

4.35 Second mesh enclosure, upstream and around the S809 blade. . . . . 78

4.36 Third mesh enclosure, downstream of S809 blade. . . . . . . . . . . . 78

4.37 Aeroelastic mesh around S809 blade, 5 deg pitch angle. . . . . . . . . 79

4.38 Aeroelastic mesh around S809 blade, 0 deg pitch angle. . . . . . . . . 79

4.39 Aeroelastic mesh inlet and outlet boundary conditions. . . . . . . . . 80

4.40 Aeroelastic mesh wall boundary conditions. . . . . . . . . . . . . 80

4.41 TSM mesh and fixed end boundary constraint. . . . . . . . . . . . 81

4.42 Aeroelastic analysis S809 blade tip deflection. . . . . . . . . . 82

4.43 S809 aeroelastic results, $V_{0}=15 \frac{\mathrm{m}}{\mathrm{s}}, \alpha=0 \mathrm{deg} \ldots \ldots \ldots 2$

4.44 S809 aeroelastic results, $V_{0}=17.3 \frac{\mathrm{m}}{\mathrm{s}}, \alpha=5 \mathrm{deg} \ldots \ldots . \ldots . . . . \quad 83$

4.45 S809 aeroelastic results, $V_{0}=19.7 \frac{\mathrm{m}}{\mathrm{s}}, \alpha=5 \mathrm{deg} \ldots \ldots \ldots$

4.46 Mesh 1 to 4 with varying element sizes. . . . . . . . . . 85

B.1 Mini-45-E transducer. . . . . . . . . . . . . . . . . . . 94

B.2 Blade dimensions . . . . . . . . . . . . . . . . . . . . . . 95

B.3 Removable section dimensions . . . . . . . . . . . . . . . . 96

B.4 LABVIEW VI program interface . . . . . . . . . . . . . 97

B.5 LABVIEW VI block diagram . . . . . . . . . . . . . . . . 98 


\section{Nomenclature}

\begin{tabular}{|c|c|c|}
\hline $\mathrm{A}$ & Rotor swept area & $\mathrm{m}^{2}$ \\
\hline$a$ & Axial interference factor & - \\
\hline$a^{\prime}$ & Tangential interference factor & - \\
\hline$\alpha$ & Angle of attack & $\operatorname{deg}$ \\
\hline$n$ & Half chord & $\mathrm{m}$ \\
\hline$\theta$ & Pitching angle & $\operatorname{deg}$ \\
\hline$x$ & Location of elastic axis & $\mathrm{m}$ \\
\hline$\beta$ & Pitch angle & $\operatorname{deg}$ \\
\hline$\varphi$ & Angle of relative wind to rotor plane & $\operatorname{deg}$ \\
\hline$\gamma$ & Angle of relative wind to rotor axis & $\operatorname{deg}$ \\
\hline$\triangle \mathrm{w}$ & Change of relative wind speed & $\mathrm{m} / \mathrm{s}$ \\
\hline$\triangle u$ & Change of wind speed & $\mathrm{m} / \mathrm{s}$ \\
\hline$\triangle v$ & Change of tangential wind speed & $\mathrm{m} / \mathrm{s}$ \\
\hline$\rho$ & Density of air & $\mathrm{kg} / m^{3}$ \\
\hline$B$ & Number of blades & - \\
\hline$b$ & Blade length & $\mathrm{m}$ \\
\hline$C_{P}$ & Power coefficient & - \\
\hline$C_{T}$ & Thrust coefficient & - \\
\hline$c$ & Chord length & $\mathrm{m}$ \\
\hline$\dot{m}$ & Mass flow rate & $\mathrm{kg} / \mathrm{s}$ \\
\hline
\end{tabular}




$\begin{array}{clc}P & \text { Power } & \mathrm{W} \\ p & \text { Pressure } & \mathrm{Pa} \\ U & \text { Torque } & \mathrm{Nm} \\ T & \text { Thrust } & \mathrm{N} \\ \sigma & \text { Solidity ratio } & - \\ \omega & \text { Angular velocity } & \mathrm{rad} / \mathrm{s} \\ u & \text { Axial velocity at rotor plane } & \mathrm{m} / \mathrm{s} \\ u_{1} & \text { Downstream velocity } & \mathrm{m} / \mathrm{s} \\ V_{0} & \text { Initial freestream velocity } & \mathrm{m} / \mathrm{s} \\ v & \text { Tangential velocity at rotor plane } & \mathrm{m} / \mathrm{s} \\ v_{1} & \text { Tangential velocity upstream } & \mathrm{m} / \mathrm{s} \\ v_{2}=v & \text { Tangential velocity downstream } & \mathrm{m} / \mathrm{s} \\ \mathrm{w} & \text { Relative windspeed } & \mathrm{m} / \mathrm{s} \\ \mathrm{w}_{1} & \text { Relative windspeed upstream } & \mathrm{m} / \mathrm{s} \\ \lambda & \text { Tip speed ratio } & - \\ r & \text { Radius to blade section } & \mathrm{m} \\ K & \text { Tip loss related factor } & - \\ \mathrm{Re} & \text { Reynold's number } & -\end{array}$




\section{Chapter 1}

\section{Introduction}

For the past decade, the demand for self-sufficiency and green energy has significantly increased along with reliance on wind energy. Industry research and the development of wind turbines has resulted in a surge of activity relating to design and optimization of horizontal axis wind turbine rotor blades to constantly achieve a greater efficiency in the energy extraction process.

\subsection{Motivation}

Efficient computational algorithms based on horizontal axis wind turbine (HAWT) aerodynamic theory are constantly developed that allow for a computer-simulated analysis of the aerodynamic and structural response of a rotor blade in a projected wind flow and predict the resulting aeroelastic response. Some aeroelastic analysis software such as the National Renewable Energy Laboratory (NREL) FAST or AeroDyn, and the less extensive Qblade software from Berlin Technical University, allow for a complete aeroelastic analysis of wind turbines throughout all stages of design and have been evaluated as reliable aeroelastic analysis algorithms. However, less refined but time efficient algorithms that provide a faster and more time-efficient solution 
that is beneficial for the early stages of analysis are also existent. These algorithms require validation against experimental data and current industry standard finite element analysis computational fluid dynamics (CFD) and finite element analysis (FEA) software such as ANSYS to ensure an acceptable degree accuracy in the preliminary stages of wind turbine blade design. The motivation of this research was to develop an appropriate methodology and process that can be used for validation of these algorithms. The development of suitable computational and experimental benchmark problems is an integral part of this. As a means of ensuring that the benchmark problems are appropriate, they must be demonstrated in their intended application. To this end, an algorithm called Preliminary Aeroelastic Analysis of Wind Turbines (PAAC-WTB), developed by McFarlane [1, 2], was selected for the purpose of this thesis.

The PAAC-WTB algorithm models the aerodynamic, structural and dynamic behaviour of any rotor blade to determine its aerodynamic performance and aeroelastic response. The aerodynamic portion of the algorithm uses the 2D Panel Method for the Blade Element Momentum Theory (BEMT) and is developed by McFarlane [2]. It determines the aerodynamic parameters and performance of the blade and consequently the rotor. In turn, the structural portion of the program integrates a kinematic beam solver which uses the Variational Asymptotic Beam Section (VABS) analysis developed by Khouli [1] to determine the blade's cross-sectional properties. Finally, using the Ritz method, a kinematic solver is developed to determine the blade's dynamic response to the air flow [2]. It should be noted that in the following chapters of this thesis, any reference to a wind turbine is meant to indicate a horizontal axis wind turbine (HAWT). 


\section{$1.2 \quad$ Objective}

The main purpose of this research was to investigate the use of experimental and computational analysis to determine the validity of the PAAC-WTB algorithm and, and by extension all similar algorithms, that was developed to correctly simulate a rotor blade's aeroelastic response while immersed in air flow at different air speeds and angles of attack. To achieve this purpose:

1. A reduced scale model blade was designed and 3D printed out of flexible laser sintered plastic using a third party vendor. The blade was used for closed-loop wind tunnel testing to collect data to validate the algorithm's accuracy against experimental data.

2. Various computational simulations were performed on the blade and the blade airfoil using the ANSYS CFD and FEA packages. Results obtained from ANSYS simulations were compared against results obtained from the algorithm to determine the algorithm's accuracy against computational data.

\subsection{Overview}

1. Chapter 2 details all literature reviewed regarding HAWT theory including actuator disc theory and the blade element momentum theory used to determine

the performance of a rotor blade. The Betz and Schmitz methods are described, which pertain to the design optimization of the twist and chord lengths of a rotor blade and its relation to the performance of the blade.

2. Chapter 3 investigates the HAWT and single blade designs analysed and compared using wind turbine analysis software QBlade, and the modelling and 
manufacturing of the S809 experiment blade. The test procedure and instrumentation used for the wind tunnel test is also described, and a comparison of results between the PAAC-WTB algorithm and experimental lift coefficient results is discussed.

3. Chapter 4 details and depicts all CFD and FEA simulations performed using the ANSYS package on the S809 airfoil and experiment blade. 2D simulations investigated the algorithm's accuracy when dealing with dynamic airfoils that have a predefined motion within the airflow. 3D simulations investigated the algorithm's ability to couple the fluid and dynamic structural solver and compare the results against the results from the two way fluid-structure interaction (FSI) analysis from the ANSYS software. 


\section{Chapter 2}

\section{Literature Review}

The purpose of this chapter is to present the relevant aerodynamic theory based on which wind turbines operate, as well as the methods used to design the rotor blade.

\subsection{Rotor Blade Aerodynamics}

The rotor blade is the most critical part of the wind turbine. Its aerodynamic properties determine the turbine's overall capability at converting wind energy into mechanical energy and in turn electrical energy. The rate at which energy is extracted
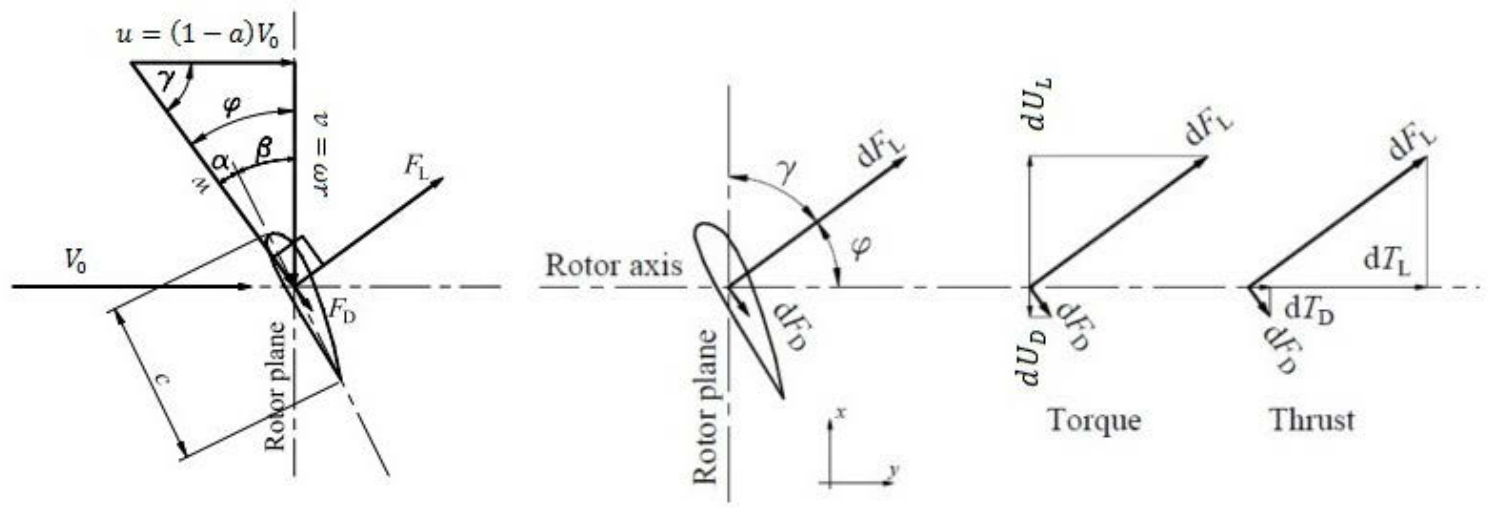

Figure 2.1: Rotor airfoil rotating in freestream flow, reproduced from [3]. 
from the incoming wind flow is defined by power $P$ :

$$
P=U \omega
$$

where $\mathrm{U}$ represents torque developed by the rotor and $\omega$ is the angular velocity of the rotor blade.

The airfoil section or sections that make up the rotor blade experience lift, drag, and axial forces as demonstrated in Figure 2.1, where $\varphi$ is the angle between the relative wind and the rotor plane, $\gamma$ is the angle between the relative wind and the rotor axis, $\beta$ is the pitch angle, $\alpha$ is the angle of attack, and $\mathrm{w}$ is the relative wind speed, i.e., the actual wind speed the airfoil element observes. All forces are applied at the airfoil's aerodynamic centre, usually located at $25 \%$ of the chord length from the leading edge. The lift force, $F_{L}$, acts perpendicular to the drag force, $F_{D}$. The resultant of those two forces is a tangential force that rotates the blade along the rotor plane. The drag force generated during the rotation of the wind turbine acts in the plane of rotation in a direction opposing rotation. Thus, it is desirable to design the blade to maximize the lift to drag force ratio in order to obtain a higher energy conversion efficiency.

The lift force is defined by the dimensionless coefficient of lift, $C_{L}$ :

$$
F_{L}=C_{L} \frac{1}{2} \rho \mathrm{w}^{2}(b c) B
$$

and the drag force is defined by the dimensionless coefficient of drag, $C_{D}$ :

$$
F_{D}=C_{D} \frac{1}{2} \rho \mathrm{w}^{2}(b c) B
$$

where $\rho$ is the density of air, $\mathrm{w}$ is relative wind speed, $b$ is the total length of the rotor blade, $c$ is the chord length of the airfoil/airfoils that make up the blade, and 
$B$ is the total number of rotor blades.

An axial thrust force is also generated in the airfoil that acts normal to the rotor's plane of rotation. It is also desirable to design the blade to be able to withstand potential thrust force as well as reduce the thrust generated in the blade.

Referring to Figure 2.1, the lift and drag forces can be transformed into torque $U$ and thrust $T$. If an airfoil section of the rotor blade of differential length $d r$ is considered, at a distance $r$ from the blade root, the differential forms of torque and thrust, $d U$ and $d T$, respectively, acting on this differential ring element can be defined as:

$$
d U=\frac{1}{2} \rho \mathrm{w}^{2} c r d r B C_{x}
$$

where

$$
C_{x}=C_{L} \sin \varphi-C_{D} \cos \varphi
$$

and

$$
d T=\frac{1}{2} \rho \mathrm{w}^{2} c d r B C_{y}
$$

where

$$
C_{y}=C_{L} \cos \varphi+C_{D} \sin \varphi
$$

It is desirable to have $C_{L}$ much greater than $C_{D}$ for blade design. 


\subsection{Actuator Disc Theory}

The actuator disc theory is a based on a one dimensional model of an ideal rotor within a control volume. The rotor is an ideal permeable disc that is frictionless with no rotation in the wake, as indicated in Figure 2.2. As wind flows across the rotor, the rotor extracts energy from the kinetic energy of the wind, which allows for rotation of the rotor and thus conversion to mechanical energy. Since energy is absorbed from the fluid, the streamlines diverge downstream of the rotor. This loss in energy results in the pressure drop, $\triangle p$, across the rotor. The resulting drag from the flow reduces the wind's speed from the freestream velocity $V_{o}$ to velocity $u$ at the rotor plane and then to velocity $u_{1}$ downstream of the rotor along the wake. Thus due to the assumption of incompressible flow, the wake downstream of the rotor expands due to the reduction in the velocity of the wind.

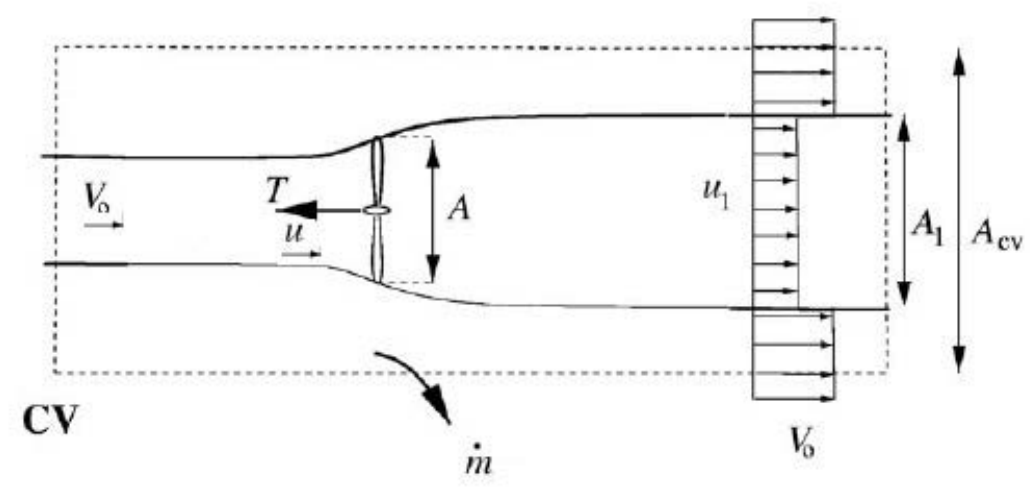

Figure 2.2: Turbine flow control volume, reproduced from [4].

Applying the principle of conservation of linear momentum actoss the rotor, the pressure drop can be defined as: 


$$
\triangle p=\rho u\left(V_{o}-u_{1}\right)
$$

and applying Bernoulli's equation across the rotor, the relation between the pressure drop and the change in velocity is:

$$
\triangle p=\frac{1}{2} \rho\left(V_{o}^{2}-u_{1}^{2}\right)
$$

Combining Equations 2.8 and 2.9 and solving for $u$, one obtains the following expression:

$$
u=\frac{1}{2}\left(V_{o}+u_{1}\right)
$$

Taking the principle of conservation of mass across the rotor, one has:

$$
\dot{m}=\rho A\left(V_{o}-u_{1}\right)
$$

and also

$$
\dot{m}=\rho u A=\rho u_{1} A_{1}
$$

where $\dot{m}$ is the mass flow rate, and $A$ and $A_{1}$ are the cross sectional areas of the flow shown in Figure 2.2. The thrust $T$ is caused by the pressure drop across the rotor plane and is assumed to be distributed uniformly across the rotor area. It is given as:

$$
T=\triangle p A
$$

Combining Equations 2.9, 2.11, and 2.13, thrust $\mathrm{T}$ can be defined as the change in momentum across the rotor disc, given as: 


$$
T=\rho u A\left(V_{o}-u_{1}\right)=\dot{m}\left(V_{o}-u_{1}\right)
$$

As mentioned previously, the actuator disc theory assumes that the flow is frictionless within the control volume. Internal energy of the system is conserved and thus the energy extracted and transferred to the shaft is determined by applying the integral energy equation to the control volume in Figure 2.2

$$
P=\frac{1}{2} \dot{m}\left(V_{o}^{2}-u_{1}^{2}\right)=\frac{1}{2} \rho u A\left(V_{o}^{2}-u_{1}^{2}\right)
$$

The axial induction factor $a$ (or interference factor) is defined as the fractional decrease in wind velocity between the freestream and the rotor plane velocity, while the tangential interference factor $a^{\prime}$ is the fractional increase in tangential wind velocity between the freestream velocity and the rotor plane velocity [3, 5]. The axial interference factor is defined by either

$$
u=V_{o}(1-a)
$$

or

$$
u_{1}=V_{o}(1-2 a)
$$

while the tangential interference factor is defined by

$$
v=r \omega\left(1+a^{\prime}\right)
$$

Substituting the axial induction factor into Equations 2.15 and 2.14, respectively, results in 


$$
P=2 \rho V_{o}^{3} a(1-a)^{2} A
$$

$$
T=2 \rho V_{o}^{2} a(1-a) A
$$

The characteristics of the rotor are defined through the power coefficient $C_{P}$ and the thrust coefficient $C_{T}$, respectively, defined as:

$$
\begin{aligned}
& C_{P}=\frac{P}{\frac{1}{2} \rho V_{o}^{3} A} \\
& C_{T}=\frac{T}{\frac{1}{2} \rho V_{o}^{2} A}
\end{aligned}
$$

Combining Equations 2.19 and 2.20 with the coefficient equations, one gets:

$$
C_{P}=4 a(1-a)^{2}
$$

and,

$$
C_{T}=4 a(1-a)
$$

The higher $C_{P}$ is, the more efficient the rotor is at extracting energy from the wind. To determine the induction factor $a$ at the maximum power coefficient $C_{P \max }$, the derivative of $C_{P}$ with respect to $a$ is determined and equated to 0 , yielding

$$
a=\frac{1}{3}
$$

which leads to a $C_{P \max }=\frac{16}{27}$ and a $C_{T \max }=\frac{8}{9}$. These maximum values are known as the Betz limit [6]. However, these are the maximum theoretical achievable values. 
Realistically, due to several unaccounted for effects such as wake rotation, drag, and root and tip losses, obtainable coefficient values are lower [4].

\subsection{Blade Element Momentum Theory}

The blade element momentum theory (BEMT) is the combination of the blade element theory (BET) derived by Glauert in 1935 [6] as well as the actuator disc theory, or momentum theory. BEMT theory allows the determination of the forces acting on a turbine disc from the change in momentum due to the motion of the fluid across the disc, and by extension determining the performance of a turbine. This theory determines the forces acting on the turbine blade by dividing the blade into twodimensional airfoil elements and calculating the generated power and thrust at each element. By integrating the generated values at each element over the span of the turbine blade, the overall performance of the blade can be determined. Utilising BEMT, one can design blade geometry to achieve desired design performance.

The BEMT makes two important assumptions:

1. Divided elements have no radial dependency, or in other words, no flow interference between elements is assumed.

2. The forces acting on each element are only due to drag and lift forces applied on that element.

Taking into consideration a finite number of turbine blades $B$, as opposed to the infinite number of blades assumption made for the momentum theory, the rotor solidity ratio $\sigma$ is defined as:

$$
\sigma=\frac{c B}{2 \pi r}
$$


where $c$ is the chord length.

Based on BEMT it can be shown that the differential torque and the differential thrust acting on a differential blade element are, respectively, given as [3]:

$$
\begin{gathered}
d U=2 \pi r^{2} \rho u v_{3} d r \\
d T=2 \pi r \rho u\left(V_{0}-u_{1}\right) d r
\end{gathered}
$$

Figure 2.3 depicts the geometric relations of the speeds in the rotor plane upstream of the rotor plane, at the rotor plane, and downstream of the rotor plane. From this figure and using Equations 2.16 and 2.18 one can define the relative windspeed $\mathrm{w}$ as:

$$
\mathrm{w}=\frac{u}{\sin \varphi}=\frac{V_{0}(1-a)}{\sin \varphi}
$$

or

$$
\mathrm{w}=\frac{v}{\cos \varphi}=\frac{\omega r\left(1+a^{\prime}\right)}{\cos \varphi}
$$

Referring back to Equation 2.27, the tangential speed downstream of the rotor plane, $v_{3}$ was used instead of the tangential speed at the rotor plane, $v_{2}$. Although there is tangential rotation of the wind, it is very small and thus makes this substitution a feasible approximation. Thus, $v_{3}$ is defined as:

$$
v_{3}=2 r \omega a^{\prime}
$$




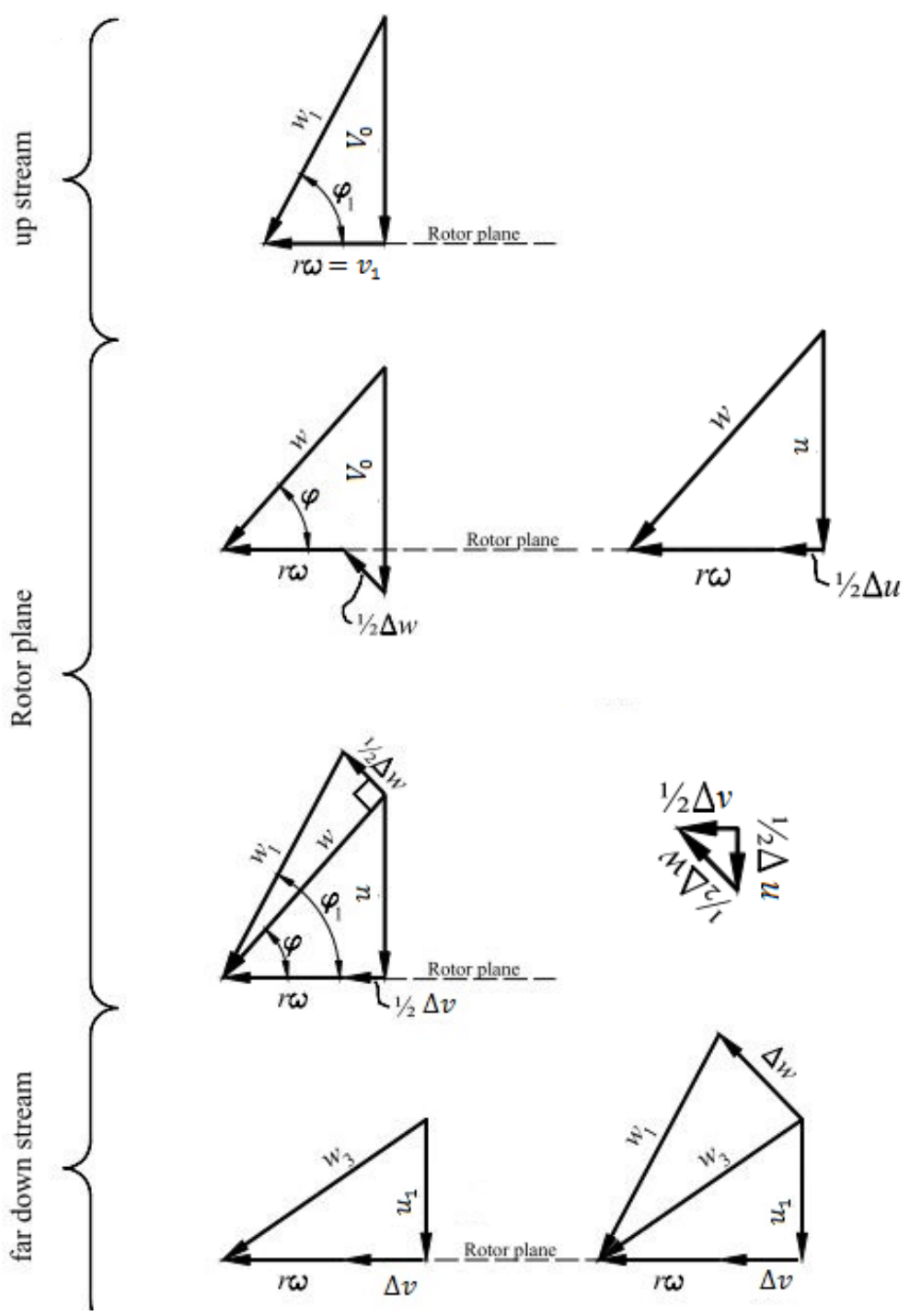

Figure 2.3: Speeds in the rotorplane defined upstream, at the rotor plane and downstream, reproduced from [3]. 
Equating 2.27 and 2.28 to Equations 2.4 and 2.6, respectively, while substituting in Equations 2.29, 2.30, 2.31, and 2.31, the following two relationships are obtained for $a$ and $a^{\prime}$ :

$$
\begin{gathered}
\frac{a}{a-1}=\frac{\sigma C_{y}}{4 \sin ^{2} \varphi} \\
\frac{a^{\prime}}{a^{\prime}+1}=\frac{\sigma C_{x}}{4 \sin \varphi \cos \varphi}
\end{gathered}
$$

The BEMT also makes the major assumption of infinite blade length, which is unrealistic and does not take into consideration the effect of shed vortices due to flow near the blade tip. Due to the relative pressure difference between the top and the bottom of the blade, wind will flow from the bottom to the top surface at the tip radially inward on the top surface as shown in Figure 2.4. This effect is characterized as tip loss. Due to the tip being farthest away from the hub, the tip loss reduces turbine efficiency and torque output.

To account for the tip loss effect, Prandtl derived a factor, known as the Prandtl tip loss correction factor [6], that should be included in the BEM equations. The factor is defined as

$$
F=\frac{2}{\pi} \cos ^{-1}\left(e^{-f}\right)
$$

where

$$
f=\frac{B}{2} \frac{R-r}{r \sin \varphi}
$$

Introducing the Prandtl tip loss factor into the interference factor relationships given by Equations 2.32 and 2.33 yields [3] 


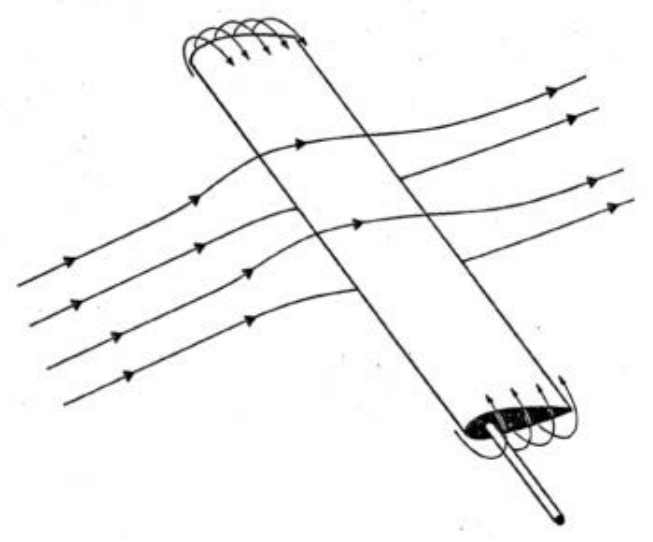

Figure 2.4: Flow over rotor blade [7].

$$
a=\frac{1}{\frac{4 F \sin ^{2} \varphi}{\sigma C_{y}}+1}
$$

and

$$
a^{\prime}=\frac{1}{\frac{4 F \sin \varphi \cos \varphi}{\sigma C_{x}}+1}
$$

Once axial interference factor values for $a$ exceed 0.2, the BEMT begins to break down iteratively and does not converge [3]. For values of a beyond 0.2, Glauert derived a different equation for the axial interference factor [3, 6]:

$$
a=\frac{1}{2}\left(2+K\left(1-2 a_{c}\right)-\sqrt{\left(K\left(1-2 a_{c}\right)+2\right)^{2}}+4\left(K{a_{c}}^{2}-1\right)\right.
$$

where

$$
K=\frac{4 F \sin ^{2} \varphi}{\sigma C_{y}}
$$

The application of BEMT is an iterative process, where $a$ and $a^{\prime}$ are repeatedly calculated for every blade element along the blade until they converge. Once this 
happens, the thrust $T$ and torque $U$ are calculated as well for every blade element and summed across the blade length to obtain the total thrust and torque generated by the rotor blades as:

$$
\begin{gathered}
T=B \int_{0}^{R} d T d r \\
U=\omega B \int_{0}^{R} d U d r
\end{gathered}
$$

\subsection{Blade Design Optimization}

For the wind tunnel tests, actual reduced scale rotor blades were required. Literature pertaining to blade design was researched, specifically the Betz's formulas and its iteration, the Schmitz's formulas were researched for optimization of the chord and pitch angle of the blade.

Detail regarding the actual design and manufacturing of the blade/blades is described in Chapter 3.

\subsection{Betz's Method}

Using the principle of conservation of linear momentum in tandem with Bernoulli's principle, the Betz limit was developed, which calculates the maximum power extracted from free stream wind flow, completely independent of the actual design.

Consider the blade in Figure 2.1 rotating in the rotor plane, with the blade split into blade elements of length $d r$ at a distance $r$ from the rotor axis, as depicted in Figure 2.5. 


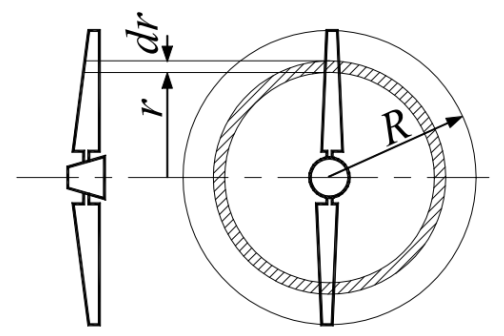

Figure 2.5: Rotor blade element [3].

Taking $n$ as the rotational speed of the rotor, the angular speed of the rotor $\omega$ is given as:

$$
\omega=2 \pi n
$$

The tip speed ratio, $\lambda$, is the ratio of the tangential speed of the blade tip, $v_{t i p}$, to the velocity of the wind, $V_{0}$. The tip speed ratio is a property of the blade that defines its efficiency that varies directly with the blade length, chord length, angle of twist, and airfoil profile. Thus,

$$
\lambda=\frac{v_{t i p}}{V_{0}}=\frac{\omega R}{V_{0}}
$$

Referring to angles $\gamma$ and $\varphi$ in Figure 2.1, combining Equations 2.42 and 2.43, and using the induction factor given in Equation 2.25, one has:

$$
\begin{aligned}
& \gamma(r)=\tan ^{-1} \frac{3 r \lambda}{2 R} \\
& \varphi(r)=\tan ^{-1} \frac{2 R}{3 r \lambda}
\end{aligned}
$$

thus, the pitch angle $\beta(r)_{\text {Betz }}$ is determined to be 


$$
\beta(r)_{\text {Betz }}=\tan ^{-1} \frac{2 R}{3 r \lambda}-\alpha_{D}
$$

where $\alpha_{D}$ is the design angle of the blade for a given design freestream velocity.

Referring to Equation 2.4, the design $C_{L}$ is usually much larger than the design $C_{D}$ for each blade element. Thus, for each blade element, Equation 2.4 reduces to:

$$
d U=\frac{1}{2} \rho \mathrm{w}^{2} c d r C_{L} \sin (\varphi)
$$

and if the power generated by each blade element is

$$
d P=d U r \omega
$$

combining Equations 2.48 and 2.47, for $B$ number of blades, leads to:

$$
d P=\frac{1}{2} B \rho \mathrm{w}^{2} c d r C_{L} \sin (\varphi) r \omega
$$

According to Betz's method, the power produced by each blade element can also be given as:

$$
d P=\left(\frac{16}{27}\right)\left(\frac{1}{2}\right) \rho V_{0}^{3}(2 \pi r d r)
$$

From Figure 2.1, $v$ is defined as:

$$
v=\mathrm{w} \sin \gamma
$$

Substituting Equation 2.51 into 2.44 leads to:

$$
V_{0}=\frac{3}{2} \mathrm{w} \cos \gamma
$$


Combining Equations 2.43, 2.51, 2.52 ,2.49, and 2.50, the optimum Betz chord length is determined as:

$$
c(r)_{B e t z}=\frac{16 \pi R}{9 B C_{L, D}} \frac{1}{\left(\lambda \sqrt{\left(\lambda^{2}\left(\frac{r}{R}\right)^{2}+\frac{4}{9}\right)}\right)}
$$

Thus the chord length for each blade element is determined based on the desired design angle of attack $\alpha_{A, D}$ and the corresponding coefficient of lift $C_{L, D}$.

\subsection{Schmitz's Method}

One major drawback to Betz's method is that the wind rotation is not considered in his model. Thus, by extension, the rotation of the wake is not taken into account, which is not ideal since the wake has an opposite rotation in comparison to the rotor downstream of the rotor plane. Schmitz further developed Betz model of the flow in order to include the effect of the wake.

Conservation of angular momentum states that the torque in the shaft is developed in reaction to the wake rotation induced in the flow downstream of the rotor. This results in a change in the relative tangential speed of the rotation at the blade. Thus, the additional tangential speed due to the average counter-rotating wake velocity is denoted by the term $\frac{1}{2} \triangle v$, resulting in

$$
v=r \omega+\frac{1}{2} \triangle v
$$

which is also defined as a function of the tangential interference factor $a^{\prime}$, as shown in Equation 2.18.

Referring to Figure 2.3, the components of the relative wind velocity w are illustrated downstream and upstream of the rotor plane as well as across the rotor 
plane. The rotational velocity of the downstream wake is $\triangle \mathrm{w}$ acting opposite to the direction of the turbine motion. Across the rotor plane, the rotational velocity will be $\frac{\triangle w}{2}$.

Assuming that $C_{L} \gg C_{D}$, then using the geometric relations in Figure 2.3, the relative wind speed and the change in the relative wind speed $\triangle \mathrm{w}$ are, respectively, given as:

$$
\begin{gathered}
\mathrm{w}=\mathrm{w}_{1} \cos \left(\varphi_{1}-\varphi\right) \\
\triangle \mathrm{w}=2 \mathrm{w}_{1} \sin \left(\varphi_{1}-\varphi\right)
\end{gathered}
$$

where $\mathrm{w}_{1}$ is the initial relative wind speed and $\left(\varphi_{1}-\varphi\right)$ is the change in the relative wind speed angle between the wind and the rotor's plane of rotation.

Using conservation of momentum, the lift force, $d F_{L}$, on each airfoil segment of the blade can be defined by the change in the relative wind velocity, $\triangle \mathrm{w}$, and the mass flow, $d q$, through the ring element (rotor) at a radius $r$ and length $d r$, i.e.,

$$
d q=2 \rho \pi r d r v
$$

and

$$
d F_{L}=\triangle \mathrm{w} d q
$$

Power was defined previously by torque $U$ and angular velocity $\omega$, therefore,

$$
d P=d U \omega=d F_{L} \sin (\varphi) r \omega
$$

where using Equations 2.57 and 2.58, we obtain the power produced for each blade 
segment as:

$$
d P=r^{2} \omega \rho 2 \pi d r \mathrm{w}_{1}^{2} \sin \left[2\left(\varphi_{1}-\varphi\right)\right] \sin ^{2}\left(\varphi_{1}\right)
$$

Taking the derivative of Equation 2.60 with respect to the relative wind speed angle $\varphi$ and equating it to 0 , the relative wind speed angle at which maximum power occurs can be determined. Solving $\frac{d P}{d \varphi}=0$, leads to

$$
\varphi=\frac{2}{3} \varphi_{1}
$$

Referring to Figure 2.3, $\varphi$ can be defined as:

$$
\varphi_{1}=\tan ^{-1} \frac{V_{0}}{\omega r}
$$

and combining Equations 2.43, 2.61 and 2.62, results in

$$
\varphi=\frac{2}{3} \tan ^{-1} \frac{R}{\lambda r}
$$

Subtracting the angle of attack $\alpha$ from the relative wind speed angle $\varphi$, the optimum blade pitch angle $\beta$, as a function of the radius of the rotor from the centre hub, is obtained

$$
\beta(r)_{\text {Schmitz }}=\frac{2}{3} \tan ^{-1} \frac{R}{\lambda r}-\alpha
$$

The optimum chord length distribution of the blade as a function of the rotor radius can be determined by considering

$$
d F_{L}=\triangle \mathrm{w} d q=\left[2 \mathrm{w}_{1} \sin \left(\varphi_{1}-\varphi\right)\right](2 \rho \pi r d r) v
$$


Taking the differential form of Equation 2.2 and using Equations 2.55 and 2.61, this results in:

$$
d F_{L}=\frac{1}{2} \rho \mathrm{w}^{2} B c d r C_{L}=\frac{1}{2} \rho \mathrm{w}_{1}^{2} B c d r C_{L} \cos \frac{\varphi_{1}}{3}
$$

Combining Equations 2.65 and 2.66 the optimum chord length function is obtained as

$$
c(r)_{S c h m i t z}=\frac{1}{B} \frac{16 \pi r}{C_{L}} \sin ^{2} \frac{\varphi_{1}}{3}=\frac{1}{B} \frac{16 \pi r}{C_{L}} \sin ^{2}\left(\frac{1}{3} \tan ^{-1} \frac{R}{\lambda r}\right)
$$

The optimum pitch angle function distributes the pitch angle along the blade such that the incoming wind intercepts the blade at the desired optimum angle of attack. The optimum chord length function ensures that the chord distribution along the blade at each segment provides maximum lift. Together the pitch angle and chord length functions determined previously then can be used to determine the optimum blade dimensions based on input parameters such as the tip speed ratio. 


\section{Chapter 3}

\section{Blade Design and Wind Tunnel Experiment}

To validate the PAAC-WTB algorithm, wind tunnel test data of the aeroelastic response of the blade was required. Lift coefficient data and strain gauge data were to be collected and compared against results obtained from the algorithm. In order to carry out the wind tunnel test, the design of rotor blades was performed for a single blade wind tunnel experiment and a HAWT wind tunnel experiment.

1. The blade had to be manufactured within a certain allowable budget

2. The experimental setup had fit within the available wind tunnel test section utilized without incurring negative aerodynamic effects such as wall blockage.

3. The blade interior was to be hollow to allow for internal installation of strain gauges. Also, the blade had to have a root interface which allows the attachment of a load cell to the blade.

4. The blade had to be reasonably flexible to allow the observation of aeroelastic effects at various wind speeds during wind tunnel testing. This required the blade material to be flexible enough and a minimum blade length of $0.5 \mathrm{~m}$. 


\subsection{Rotor Blade Design}

The wind tunnel testing was planned as two phases. The first phase involved a preliminary single blade wind tunnel experiment of a non-rotating single rotor blade immersed in air flow at various speeds and angles of attack. The second phase involved a preliminary design of a HAWT rotor blade. The purpose of the wind tunnel testing was to observe the aeroelastic effects the blades experience and collect experimental validation data for comparison against the PAAC-WTB data.

\subsubsection{Single Blade Design and Manufacturing}

Several airfoils were researched for this phase, and a few appropriate National Renewable Energy Laboratory (NREL) wind turbine airfoils were selected: the S833, S834, S835, and the S809 airfoils. All airfoil profiles are shown in Figures 3.1, 3.2, 3.3, and 3.4. The availability of published wind tunnel data for the S809 as a single non-rotating blade from several different wind tunnel experiments during the last decade, made it a good candidate for the single blade wind tunnel experiment [8-10]. The S833, S834, and S835 were selected for the HAWT wind tunnel experiment.

The S809 airfoil was designed as a member of the large blade, thick airfoil family of blades for spans of 10 to $15 \mathrm{~m}$ by NREL in 1986. These blades were designed for rotors with a power rating range of 100 to $400 \mathrm{~kW}$. The S809 has a maximum lift coefficient of $C_{l, \max }=1.0$ and is utilized on large wind turbine blades as a precursor to a tip region airfoil [11]. It was also designed to obtain a restrained maximum lift coefficient that does not decrease in value due to transition near the leading edge on the top and bottom surface of the airfoil, which explains the unique asymmetric shape of the airfoil as well as the unique trailing edge. The S809 also has low profile drag in the lift coefficient range of 0.2 to 0.8 at a Reynolds number of $2.0 \times 10^{6}$. To 


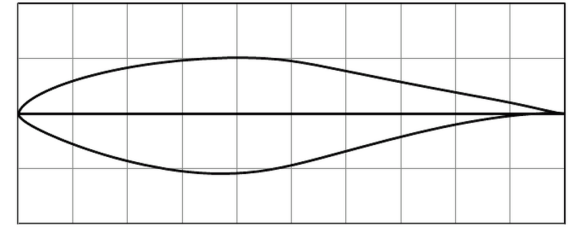

Figure 3.1: S809 airfoil [13].

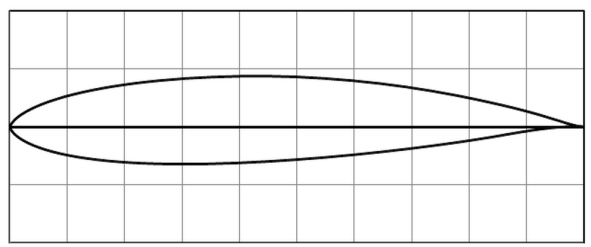

Figure 3.3: S834 airfoil [15].

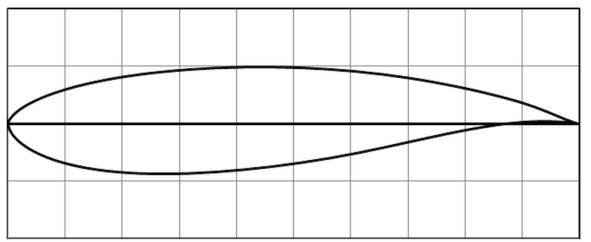

Figure 3.2: S833 airfoil [14].

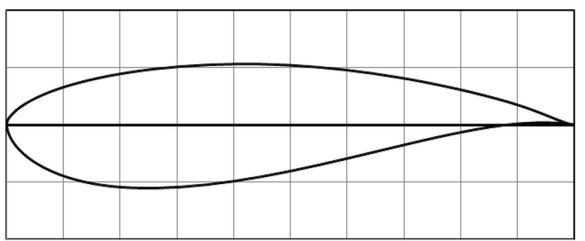

Figure 3.4: S835 airfoil [16].

achieve this the maximum airfoil thickness occurs at 21 percent chord length [12].

The reduced S809 blade was designed, utilising the Creo 3D solid modelling software. This allowed for an iterative process, where the blade design was influenced by the wind tunnel experimental setup and test plan. The final design adopted is illustrated in Figures 3.5 and 3.6. Full dimensions of the blade are provided in Appendix B.
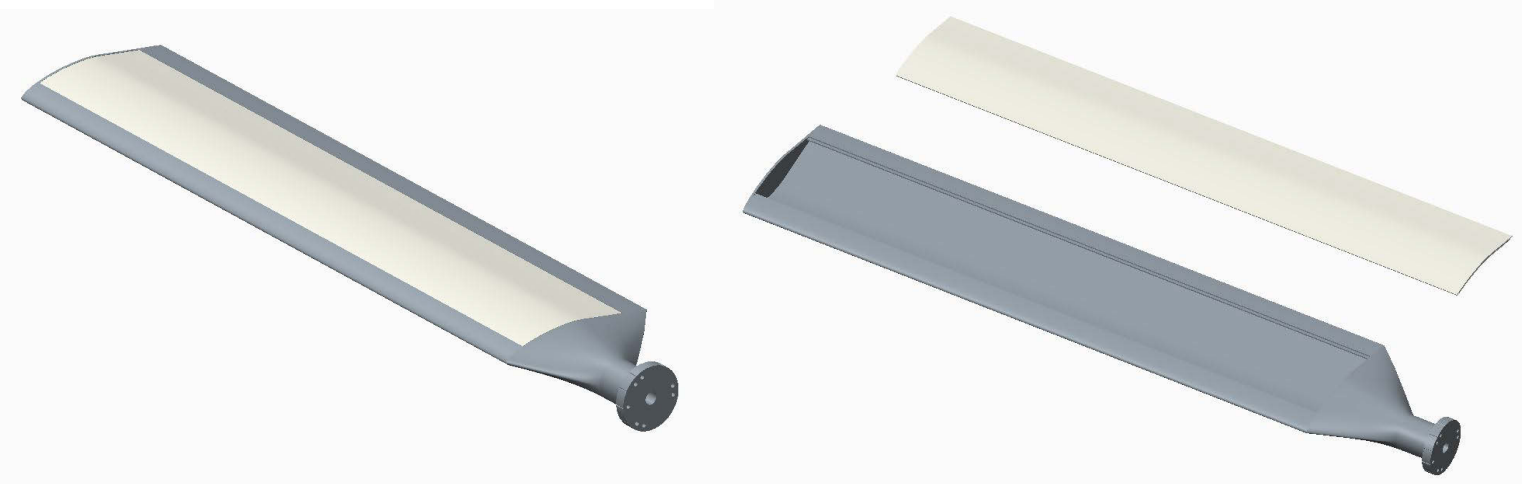

Figure 3.5: S809 blade shaded model. Figure 3.6: Blade with removable section. 
The blade length was selected to be $0.65 \mathrm{~m}$. This was to ensure that the blade fit within the closed loop wind tunnel dimensions of $0.71 \mathrm{~m}$ width and $0.81 \mathrm{~m}$ height, while also allow for observable aeroelastic response during the wind tunnel test. Using ANSYS Workbench, an analysis was performed on the S809 blade at a wind speed of $15 \frac{m}{s}$ to determine the tip deflection at the lowest selected experiment wind tunnel speed. A CFD analysis was first performed on the blade. The resulting pressure distribution from the airflow was then imported into the ANSYS structural module to perform an FEA analysis. Figure 3.7. Results show a maximum tip deflection of $0.012 \mathrm{~m}$. In order to determine if this was sufficient, the blade's reduced frequency at that speed was assessed using aeroelastic parameter $k$, which defines the potential unsteadiness of an airfoil when immersed in fluid flow. Based on the frequency of the tip deflection of the blade, the reduced frequency was determined to be approximately 3, which indicates a high degree of unsteadiness. Hence a considerable aeroelastic response was to be expected during wind tunnel testing. Reduced frequency is further discussed in Chapter 4
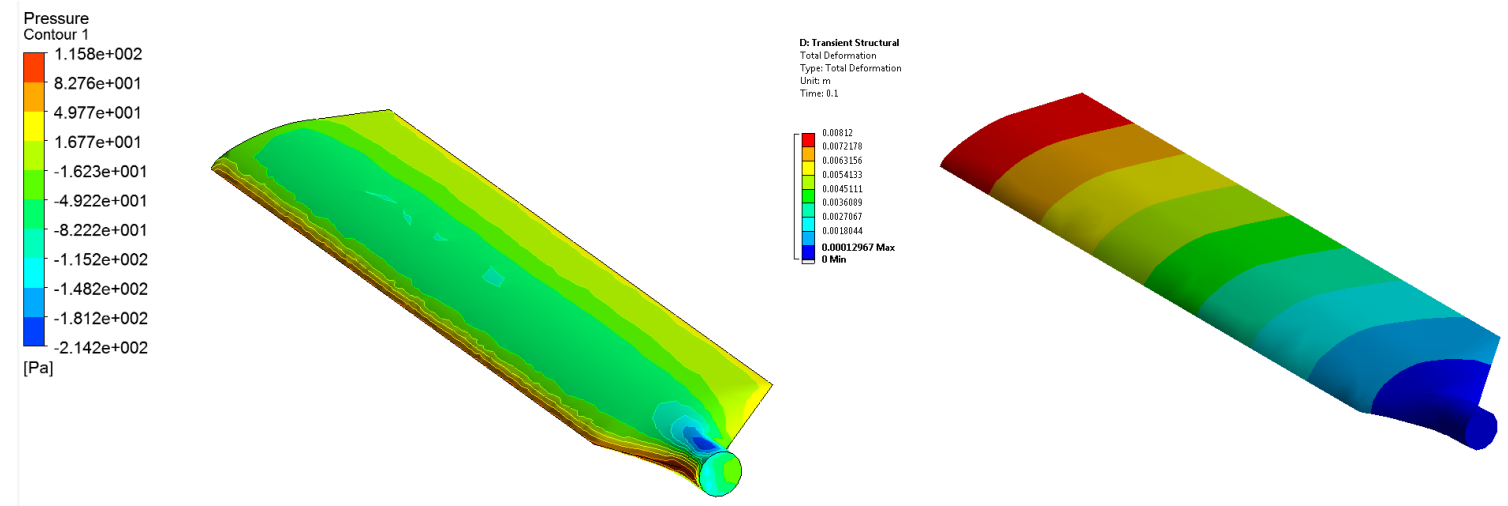

Figure 3.7: Pressure distribution (left) and structural deformation (right) of S809 blade at $15 \frac{\mathrm{m}}{\mathrm{s}}$.

To allow for internal installation of strain gauges, the blade was designed hollow 
with a wall thickness of $2 \mathrm{~mm}$, and one of the blade surfaces was designed as a removable surface, illustrated in Figure 3.6. The root portion of the blade was designed with a taper to allow for wind tunnel installation. The tapered portion contains a hollow tube along its length to serve as a conduit for the installed strain gauge wires through and to avoid aerodynamic obstruction during testing. The bottom of the root consists of the load cell interface onto which the load cell is attached, shown in Figure 3.8. More detail regarding the strain gauges and the load cell is provided later in this chapter.

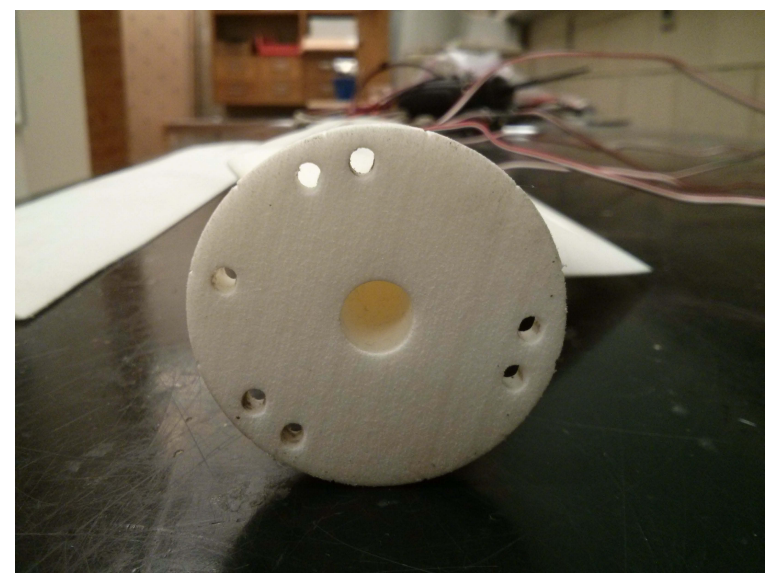

Figure 3.8: Load cell interface.

To produce a blade with a hollow interior, composite manufacturing was considered using epoxy resin and fiberglass. Several manufacturing methods were evaluated including wet layup, vacuum resin transfer molding, and oven-based inflation bladder moulding. Initial design and modelling of a clamshell mould for the inflation bladder moulding method was created; however, it was not possible to utilize the computer numerical control CNC machining facilities at Carleton to manufacture it due to size constraints. Wet layup and vacuum resin transfer moulding was also not feasible due to the lack of a ventilated composite manufacturing room at the time of manufacture. Manufacturing the composite blade at a third party vendor was also considered but 
pricing exceeded the allotted budget, and the idea was discarded.
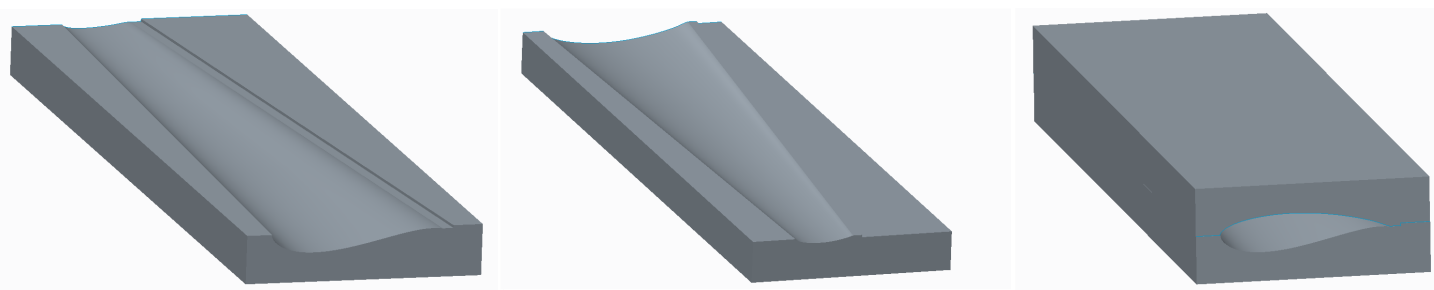

Figure 3.9: Clamshell mould.

Thus an alternative option was to 3D print the complete blade, with laser sintered plastic PA2200 material. Shapeways, a vendor based in Seattle, was selected to print the S809 blade. A printing constraint of $660 \mathrm{~mm}$ total length was advised by Shapeways, thus the S809 blade length of $650 \mathrm{~mm}$ was determined to be acceptable.

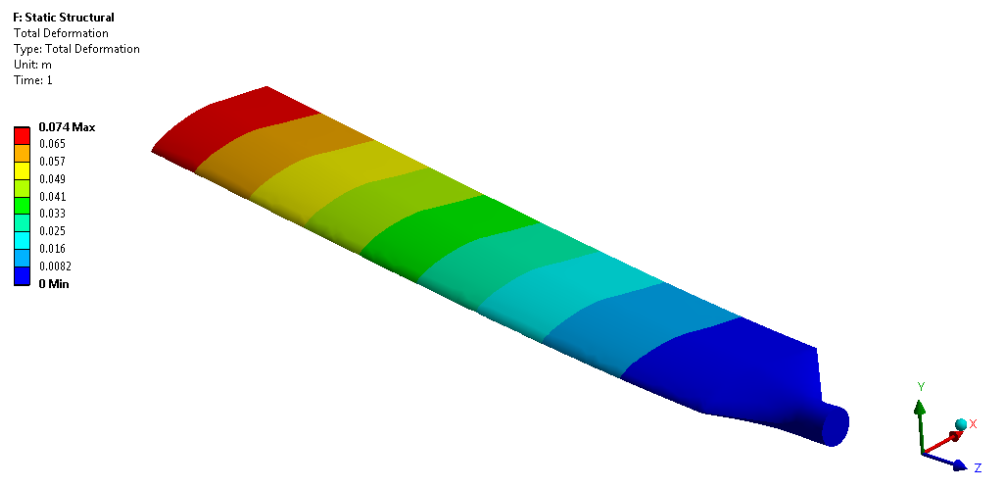

Figure 3.10: Blade deflection for $V_{0}=55 \frac{\mathrm{m}}{\mathrm{s}}$. 


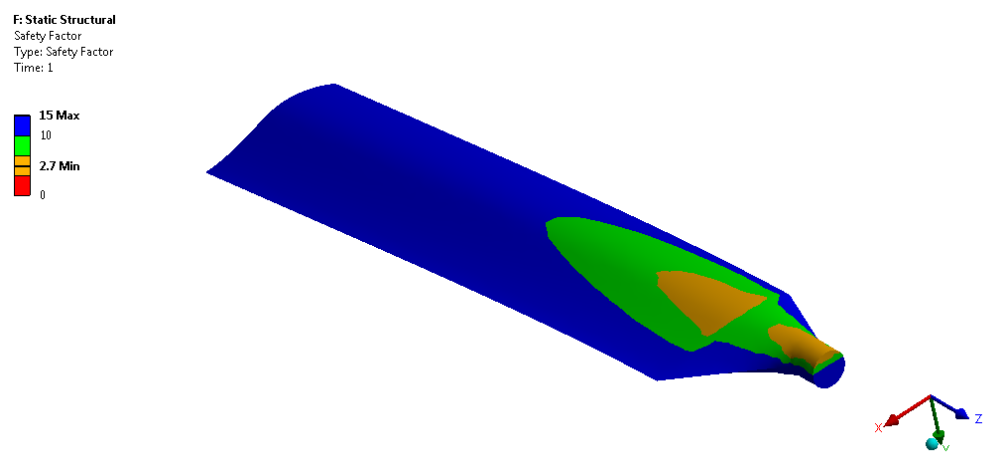

Figure 3.11: Safety factor for $V_{0}=55 \frac{\mathrm{m}}{\mathrm{s}}$.

Prior to printing the blade, a rudimentary FEA analysis using ANSYS Workbench was performed on the blade model with PA2200 as the assigned material. This was to assess the blade's ability to withstand the desired experiment wind tunnel speeds without failure. A dynamic pressure of $1851.3 \mathrm{~Pa}$ was applied to the bottom surface of the blade to simulate worst case conditions, with the blade surface perpendicular to the flow, at maximum wind tunnel speed of $55 \mathrm{~m} / \mathrm{s}$.

Table 3.1: PA2200 material properties .

\begin{tabular}{|c|c|}
\hline Parameter & Value \\
\hline Density $\rho$ & $930 \frac{\mathrm{kg}}{\mathrm{m}^{3}}$ \\
\hline Young's Modulus E & $1700 \mathrm{MPa}$ \\
\hline Tensile strength & $48 \mathrm{MPa}$ \\
\hline Poisson's ratio & 0.4 \\
\hline
\end{tabular}

Figure 3.10 shows the maximum deformation of the blade while Figure 3.11 shows the safety factor of the blade under the applied load. The blade experienced a maximum deflection of $0.074 \mathrm{~m}$ under the load thereby achieving a safety factor of 2.7 against yield strength of the material. Thus, the PA2200 material was deemed suitable for the blade. PA2200 material properties are listed in Table 3.1 


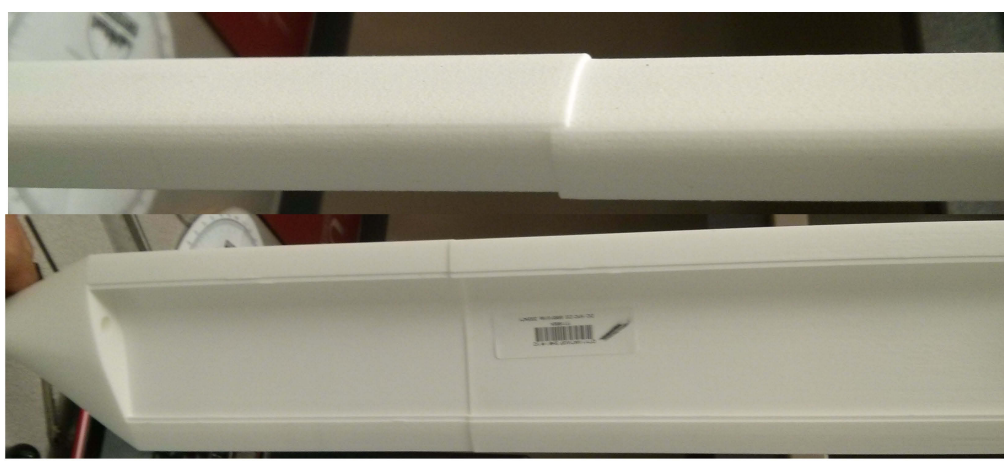

Figure 3.12: Printed models with defects.

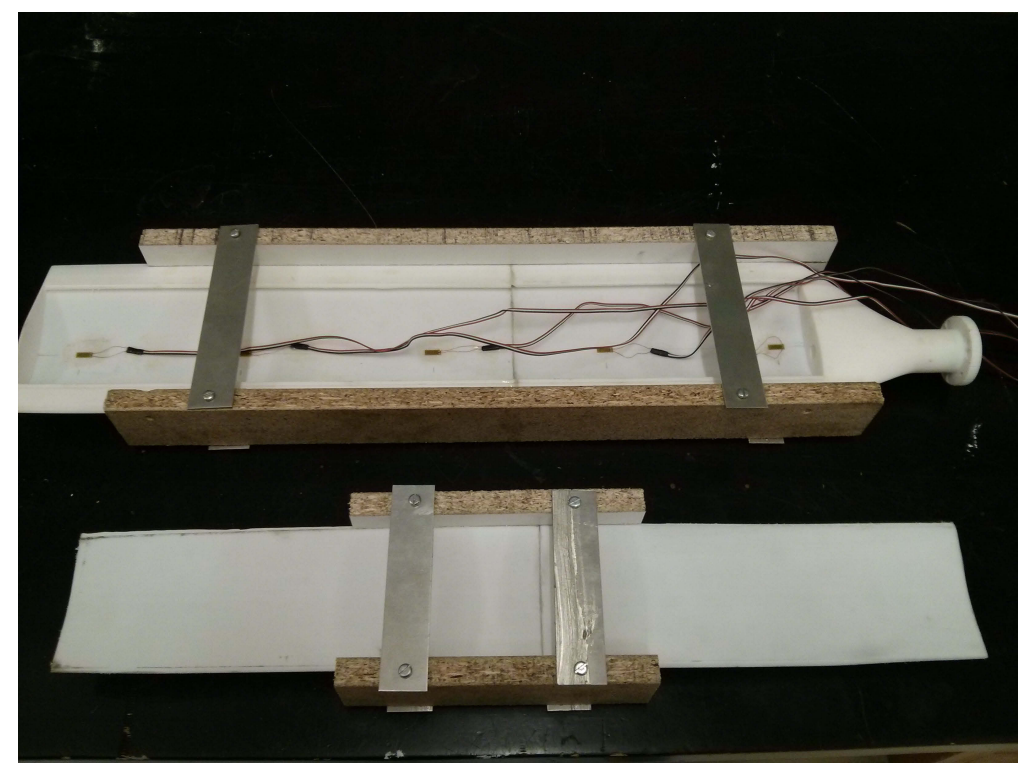

Figure 3.13: Alignment jigs.

The blade and top section were both printed and received; however, with unexpected defects along the centre, as shown in Figure 3.12. After inquiry, it was concluded by Shapeways that the defect is a laser misalignment issue that occurs during the printing of the large pieces. The solution that was adopted was to remove the defective section and re-attach the two parts utlizing epoxy resin. This was considered as a viable solution, since the epoxy resin allows for a structurally-sound bond once cured.

Both printed segments were cut using a bandsaw to remove the defects and an 


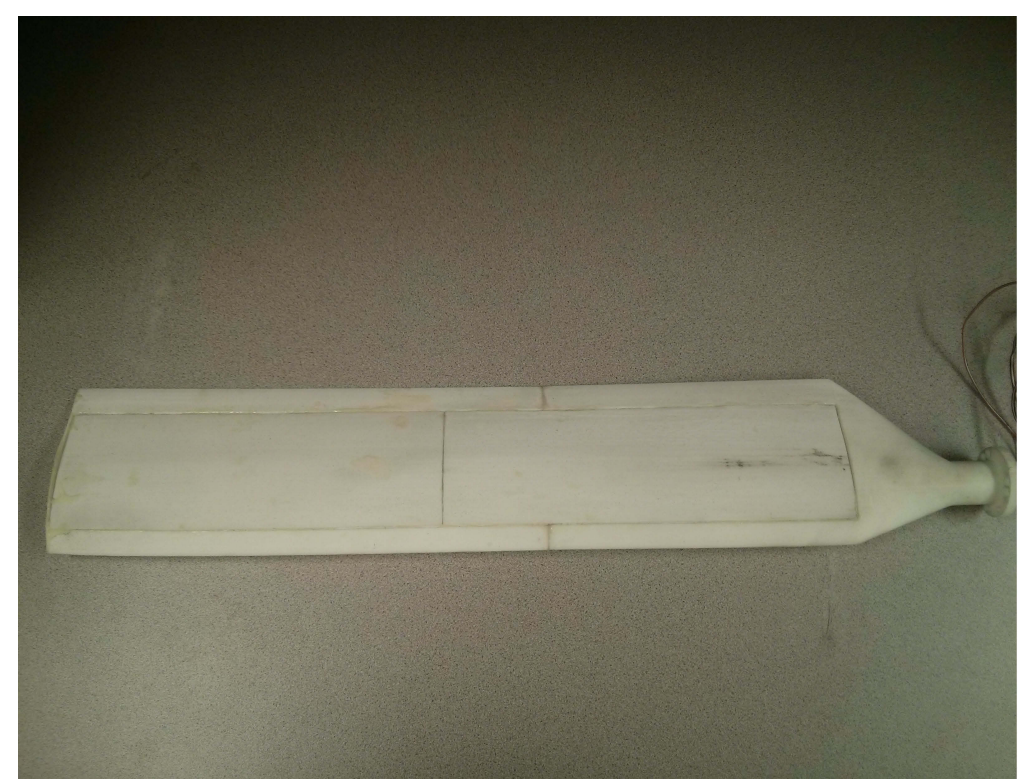

Figure 3.14: Assembled blade.

alignment jig for each printed model was manufactured to allow for accurate alignment of the two halves during bonding. Figure 3.13 illustrates the printed segments in their alignment jigs. Both halves were inserted into the jigs and a five minute epoxy resin was applied to the faces of the connecting halves. The surfaces to which the epoxy was applied were thoroughly sanded to ensure cleanliness and smoothness for maximum effective bonding surface area. The models were then left to cure for 48 hours to ensure the epoxy had retained its complete cured state strength properties [17].

Upon curing completion, 5 Vishay Micro-Measurements C2A-062LW-120 strain gauges were installed inside the blade along the midpoint of the lower surface. The gauges were set $13.5 \mathrm{~mm}$ apart. The strain gauge wiring was then routed along the blade root opening and the top removed section of the blade was bonded to the blade, again utilizing five minute epoxy resin. Strain gauges installed are shown in Figure 3.13. The final assembled blade is shown in Figure 3.14. 


\subsubsection{HAWT Blade Design}

For the HAWT design, a hybrid blade consisting of S833, S834, and S835 airfoils was selected. Together, these make up an airfoil suitable for $1 \mathrm{~m}$ to $3 \mathrm{~m}$ diameter horizontal axis wind turbines. They are characterized by low noise, thick camber, and high efficiency in laminar flow. All three airfoils have a high lift to drag ratio and insensitivity to roughness at their leading edge [18].

The S835 is the thickest of all three, thus making it the airfoil of choice for the root portion of the blade. The S833 is the second thickest; thus it was selected as the primary airfoil. The S834 is the least thick of the three and was thus selected for the tip portion of the blade.

Two blade designs were considered utilizing all three airfoils as shown in Figures 3.16 and 3.15. These designs were developed utilizing the wind turbine analysis software QBlade [19]. The blade design interface allows for the design of hybrid blades that include several types of airfoils. It also offers blade optimization options based on the Betz and Schmitz methods described in Chapter 2. Table 3.2 describes the aspects of the designed blades. Both blades were designed with a length of 0.65 m.

Table 3.2: Description of blade designs.

\begin{tabular}{|c|c|c|c|}
\hline Blade & Airfoil & $\begin{array}{c}\text { Twist } \\
\text { optimization }\end{array}$ & $\begin{array}{c}\text { Chord } \\
\text { optimization }\end{array}$ \\
\hline Hybrid linear blade & $\begin{array}{c}\text { root }=\mathrm{S} 835 \\
\text { main }=\mathrm{S} 833 \\
\operatorname{tip}=\mathrm{S} 834\end{array}$ & $\begin{array}{c}\text { Linear } \\
\alpha=5 \mathrm{deg}\end{array}$ & Linear taper \\
\hline Hybrid Schmitz blade & maot $=\mathrm{S} 835$ & Schmitz & S833 \\
& tip $=\mathrm{S} 834$ & $\alpha=0$ deg & Schmitz \\
\hline
\end{tabular}




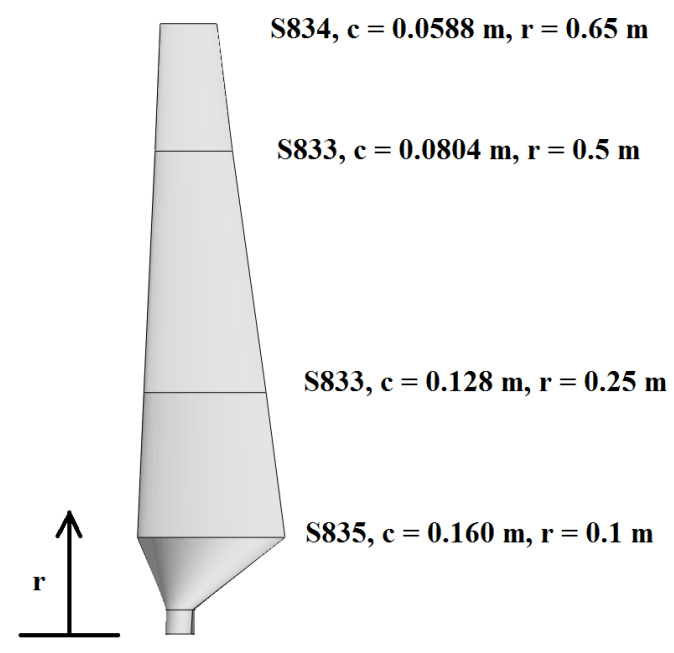

Figure 3.15: Hybrid Schmitz blade.

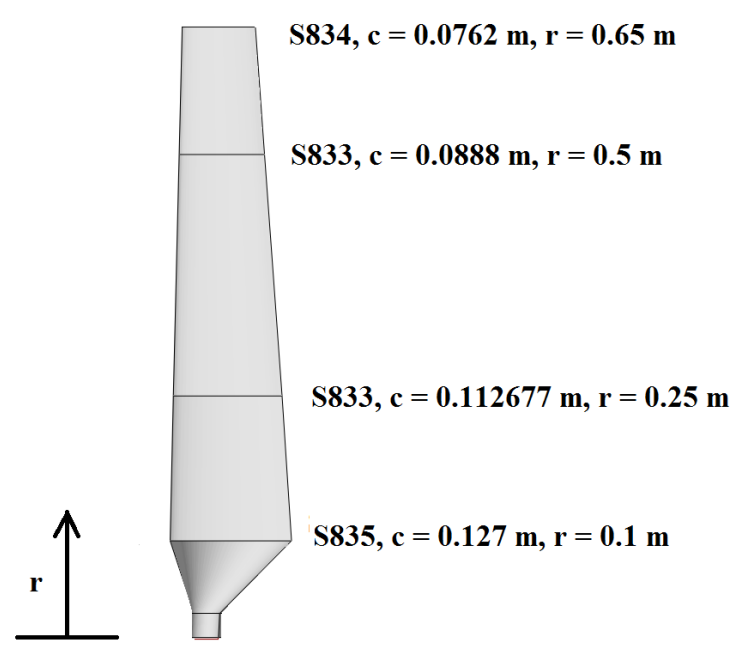

Figure 3.16: Hybrid linear blade.

The taper and twist of a wind turbine blade serve specific purposes. The taper of the blade, where the chord is largest at the root and smallest at the tip, is due to the varying bending load along the blade, with the maximum bending load applied at the root. Since the relative speed of the tip of the rotor blade is much higher in comparison to the root of the rotor blade, the apparent wind velocities are different along the blade. As such, to maintain optimum lift force, the blade is twisted along its length to change the apparent angle of attack of the incoming air flow [4].

Qblade also integrates XFOIL into the analysis, which is a widely-used opensource code specifically designed for the design and analysis of subsonic isolated 2D airfoils using input parameters such as the airfoil coordinates, the Reynolds number, and the Mach number of the flow [19]. XFOIL is able to calculate the pressure distribution along the airfoil on both top and bottom surfaces, and therefore calculate the lift and drag coefficients of the airfoil. Along with the BEMT, QBlade can determine the characteristic performance of a blade.

A rotor analysis for each blade was performed. Each rotor consists of three blades with a hub radius of $0.1 \mathrm{~m}$ each. This analysis was done using QBlade employing 


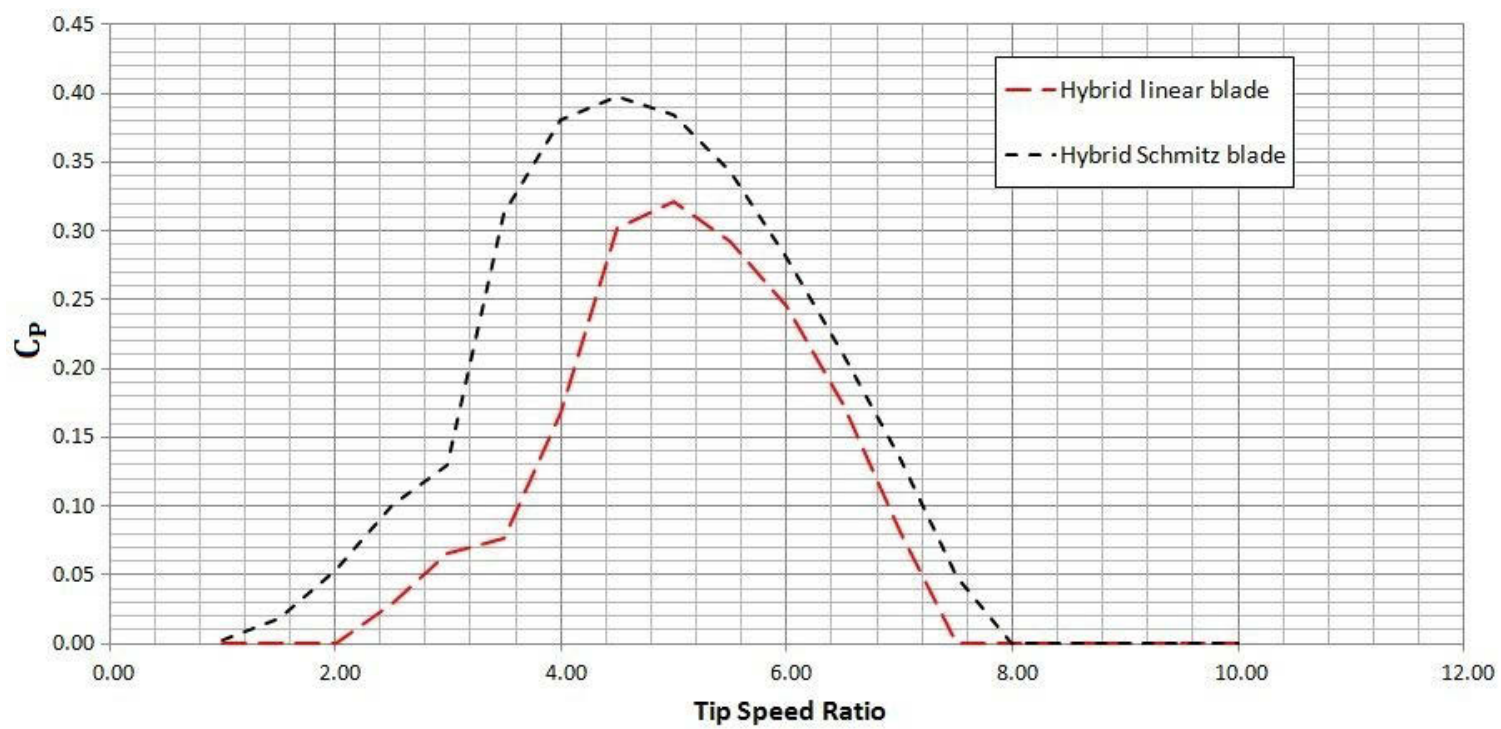

Figure 3.17: Power coefficient versus tip speed ratio.

the BEMT method, which is described in detail in Chapter 2. The power and thrust output of each blade is obtained and plotted for comparison.

Using Equations 2.21, 2.22, and 2.43 from Chapter 2, the power coefficient $C_{P}$ and the thrust coefficient $C_{T}$ can be defined as a function of the tip speed ratio $\lambda$. This leads to:

$$
\begin{aligned}
& C_{P}=\frac{2 P \lambda^{3}}{\rho R^{3} \omega^{3} A} \\
& C_{T}=\frac{2 T \lambda^{2}}{\rho R^{2} \omega^{2} A}
\end{aligned}
$$

Figures 3.17 and 3.18 illustrate the power coefficient $C_{P}$ and thrust coeffcient $C_{T}$, respectively, of each rotor at various tip speed ratios $\lambda$. As expected, the hybrid blade optimized for chord and twist utilizing the Schmitz method displayed better power performance compared to the hybrid linear blade, which has a Schmitz-optimized twist but a linear chord distribution. As outlined in Chapter 2, the thrust force T 


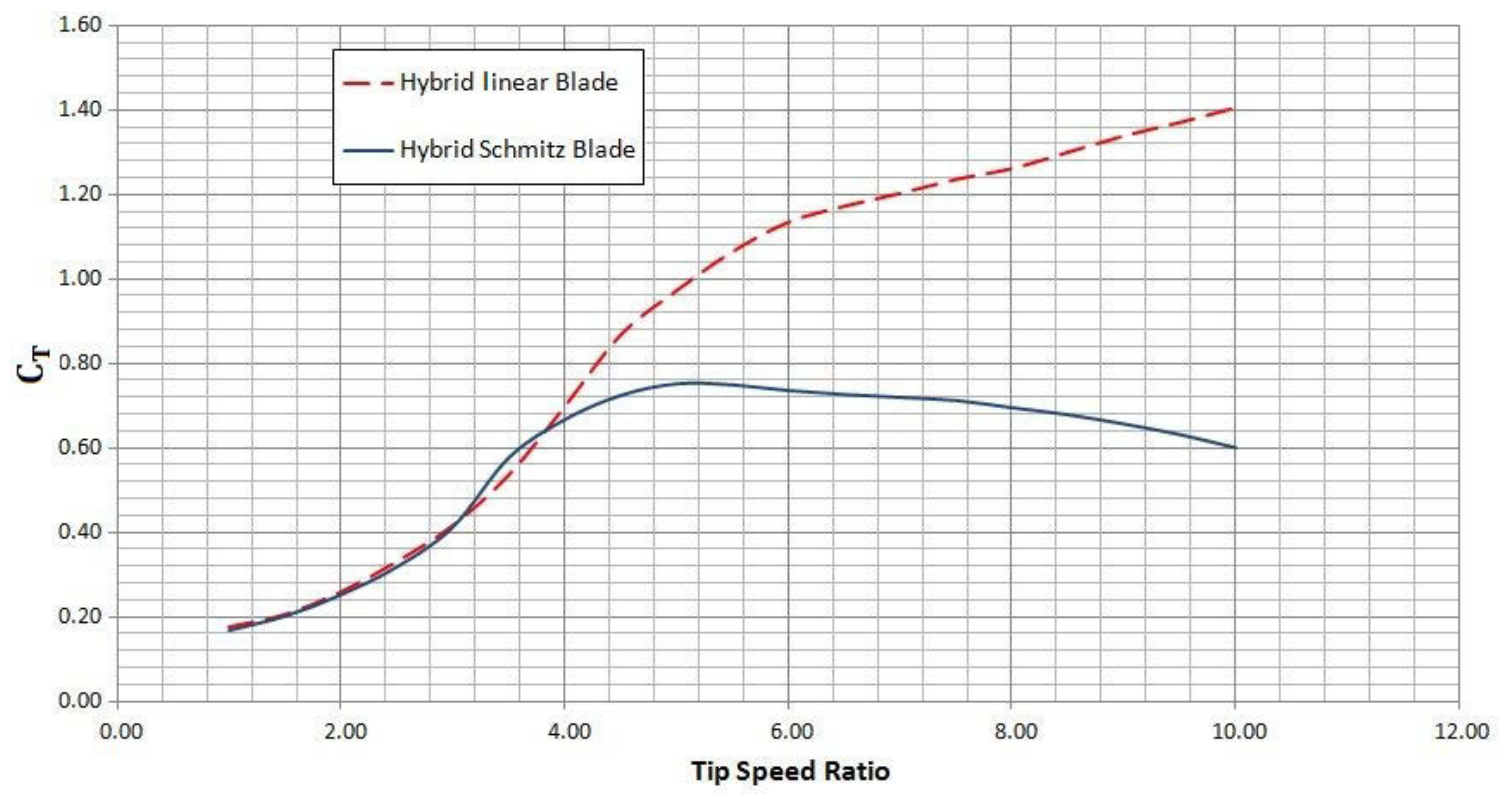

Figure 3.18: Thrust coefficient versus tip speed ratio.

applied on the rotor blades is due to the drop in pressure across the rotor plane. Figure 3.18 shows that the hybrid Schmitz blade experiences a lower thrust across tip speed ratios when compared to the hybrid linear blade. This is due to the linear chord distribution of the Hybrid linear blade, which causes the blade to experience a larger thrust force due to the larger chord lengths towards the tip of the blade. Thus, the hybrid Schmitz blade was selected for the HAWT experiment.

The preliminary design of the HAWT, shown in Figure 3.19, was modelled utilizing Creo 3D solid modelling. The atmospheric boundary layer wind tunnel at Carleton University, with a width of $1.67 \mathrm{~m}$ and a height of $1.20 \mathrm{~m}$, was evaluated to determine if the experiment can be performed without suffering significant wind tunnel blockage effects. The blockage effect occurs when an object is present in a closed test section that causes an acceleration in the flow around the object to maintain the flow's continuity. This results in larger forces applied on the body when compared to free stream conditions $[20,21]$. The blockage ratio is given as: 


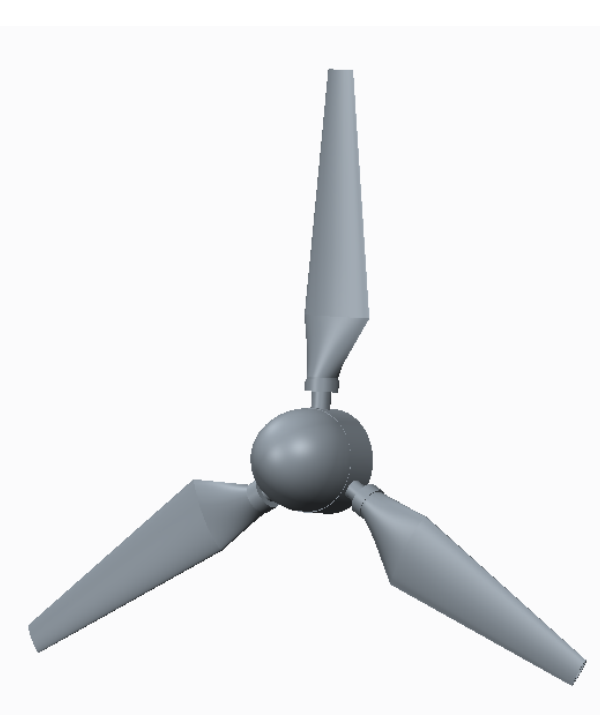

Figure 3.19: Preliminary HAWT design model.

$$
\text { Blockage }=\frac{\mathrm{A}_{\text {swept }}}{\mathrm{A}_{\mathrm{x}}} \times 100
$$

Where $A_{\text {swept }}$ is the swept area of the rotor and $A_{x}$ is the wind tunnel crosssectional area. Related literature concludes that, based on experimental results, a blockage ratio of $15 \%$ is acceptable, beyond which correction factors are required $[22,23]$. It was determined that the hybrid linear and hybrid Schmitz blades at their current dimensions, shown in Figures 3.15 and 3.16, will suffer a blockage ratio of approximately 90\%. Reduction in blade length was considered; however, reducing the length of the blade would cause an increase in blade stiffness. This would significantly hamper the HAWT blades' aeroelastic response during testing, which conflicts with the experiment's requirement to observe and collect validation data of the HAWT experiencing aeroelastic effects. Thus, after further investigation, further work on the HAWT design, manufacturing and experimental procedure development was halted due to the lack of a sizeable wind tunnel to perform the HAWT experiment. 


\subsection{Wind Tunnel Experiment}

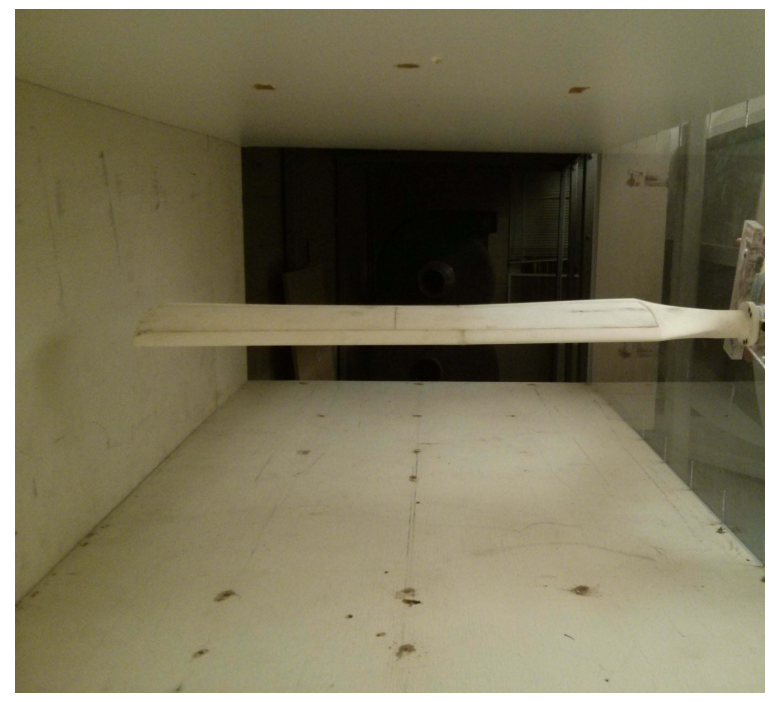

Figure 3.20: S809 blade installed in the test section.

The wind tunnel experiment on the single non-rotating blade was performed in the Carleton University high-speed wind tunnel. It has a maximum operational air speed of $55 \mathrm{~m} / \mathrm{s}$. This wind tunnel is a closed-circuit wind tunnel with an open test section. Air from the fan circulates around the tunnel loop continuously with very little or no exposure to external air. Corner turning vanes and screens through the section are used to control flow quality and reduce turbulence at sharp corners [21].

The blade was attached to a rotatable plate with an attached protractor which was then secured to the test section wall using $0.05 \mathrm{~m} \mathrm{x} 0.15 \mathrm{~m}$ planks and threaded rods. Using the rotatable plate, the required angle of attack was set manually between test runs. The data acquisition instrumentation used was set adjacent to the wind tunnel in a secure manner to avoid any potential interference with the experiment. Figures 3.20 and 3.21 show the experimental setup of the blade. Figure 3.22 shows the DAQ setup. 


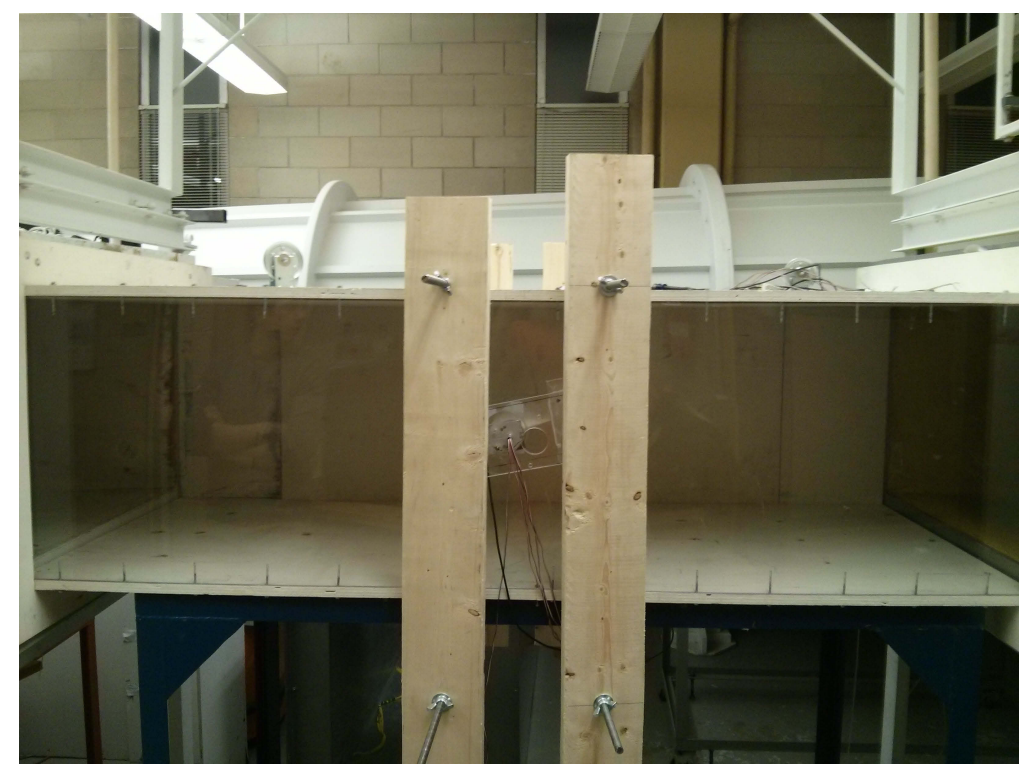

Figure 3.21: Test section in position in the closed loop wind tunnel.

Table 3.3: Wind tunnel test trials.

\begin{tabular}{|c|c|c|}
\hline Angle of Attack (deg) & Fanspeed (rpm) & Windspeed (m/s) \\
\hline$-10 \mathrm{deg}$ & 400 & 17.3 \\
\hline$-10 \mathrm{deg}$ & 500 & 19.7 \\
\hline $0 \mathrm{deg}$ & 400 & 17.3 \\
\hline $0 \mathrm{deg}$ & 500 & 19.7 \\
\hline $10 \mathrm{deg}$ & 400 & 17.3 \\
\hline $10 \mathrm{deg}$ & 500 & 19.7 \\
\hline
\end{tabular}

Experimental data was collected at three angles of attack with two different windspeeds at each angle. Windspeed is controlled by increasing or decreasing the fan's rotational speed (rpm). Thus to determine the speed in $\mathrm{m} / \mathrm{s}$, a handheld rotating vane anemometer HHF141A from Omega Engineering was used. Table 3.3 shows the experimental parameters.

Data collected consisted of load cell readings of force and torque from the six-axis 


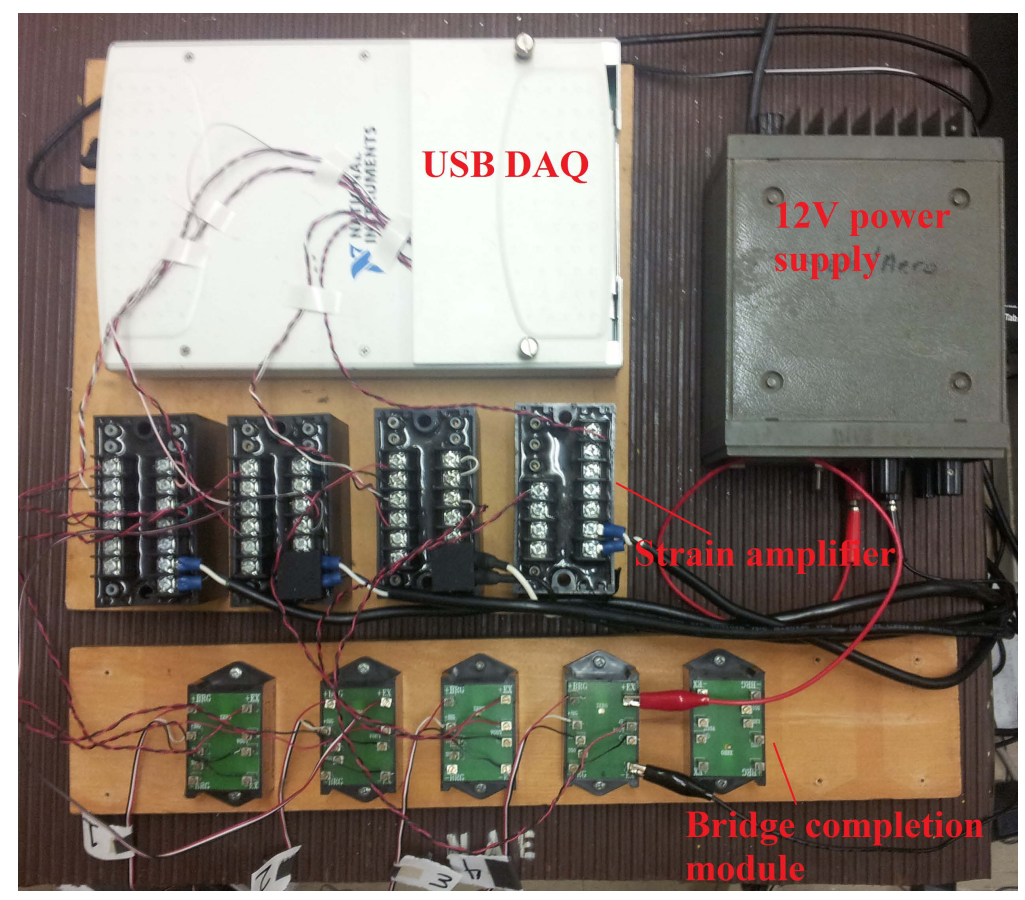

Figure 3.22: Data acquisition instrumentation.

load cell installed at the root of the $\mathrm{S} 809$ blade, and strain readings from the strain gauges installed along the blade. Data acquisition was performed on a standard desktop computer with a Core i5 processor. The NI-6034E PCI DAQ card was installed into the desktop and connected to the load cell for load data acquisition. The strain gauge wire leads were connected to the NI-6251 USB DAQ using the appropriate screw terminals, to collect strain data. A complete list of the instrumentation used is provided in Table 3.4. Using the accompanying NI LABVIEW software, a program was prepared in LABVIEW to collect both load cell readings and strain gauge readings from the connected DAQs. The program along with the interface are shown in Appendix B. Data was collected at a sampling rate of $1 \mathrm{kHz}$ which includes the frequency range of the first two bending modes of the blade. 
Table 3.4: Wind tunnel experiment data acquisition instrumentation.

\begin{tabular}{|c|c|}
\hline Instrumenation & Brand/Supplier \\
\hline Load cell & $\begin{array}{c}\text { ATI Mini45-E transducer } \\
\text { /ATI Industrial Automation }\end{array}$ \\
\hline Strain gauges & $\begin{array}{c}\text { Vishay Micro-Measurements } \\
\text { C2A-063LW-120 ohm/Intertechnology }\end{array}$ \\
\hline Strain amplifiers & DMD-465WB/Omega Engineering \\
\hline Bridge completion units & BCM-1/Omega Engineering \\
\hline USB DAQ & NI-6251 screw terminal/National Instruments \\
\hline PCI DAQ & NI-6034E DAQ card/National Instruments \\
\hline
\end{tabular}

\subsubsection{Wind Tunnel Experiment Results}

The load cell readings and strain gauge readings were processed and analysed to reduce signal noise. An averaging filter was applied in the LABVIEW program to reduce the noise with minor success. Typical filtered load cell data is shown in Figure 3.23. Ultimately strain gauge readings were deemed insufficient and unreliable. Two of the strain amplifiers malfunctioned and the remaining three amplifiers gave inaccurate strain readings that fluctuated too erratically. This is attributed to the amplifier's tuning process for variables such as signal gain, which only provides an estimate of the desired gain at best.

The load cell data was compared against results produced from the PAAC-WTB algorithm using the same wind tunnel experimental trial parameters and conditions. Data from both sources were converted into the frequency domain using the Fast Fourier Transform (FFT) method in MATLAB [2]. The resulting frequencies are shown in Figures 3.24 through 3.29. 


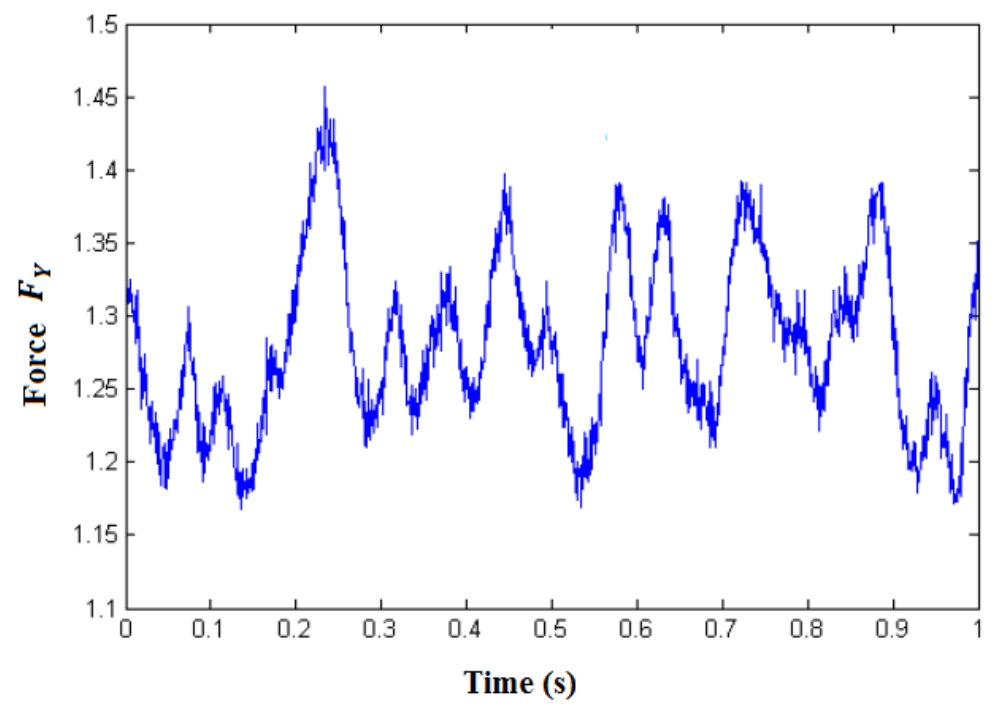

Figure 3.23: Filtered load cell data.

Lift results from the algorithm show partial agreement with the experimental results obtained. The first bending mode peak from the PAAC-WTB algorithm somewhat corresponds to the first bending mode peak from the blade load cell data. Modal analysis of the blade performed using ANSYS, presented in Chapter 4, shows lower natural frequencies when compared to the natural frequencies predicted by the algorithm. One potential conclusion is that the algorithm tends to over predict the frequencies, hence the discrepancy for the first bending mode frequency. The algorithm does not consider damping nor does it include turbulence modelling, both of which exist in the wind tunnel experiment, which may also explain the higher frequency values predicted by the algorithm. Some peaks may be due to deflections in the blade support, which consists of the planks and the plexiglass rotatable base, both of which may have experienced excitation due to the deflection of the blade. Nevertheless, the algorithm was able to predict some aspect of the preliminary experiment results, which provides a basis for further experimental testing and data acquisition that should be performed in the future to obtain more validation data. 


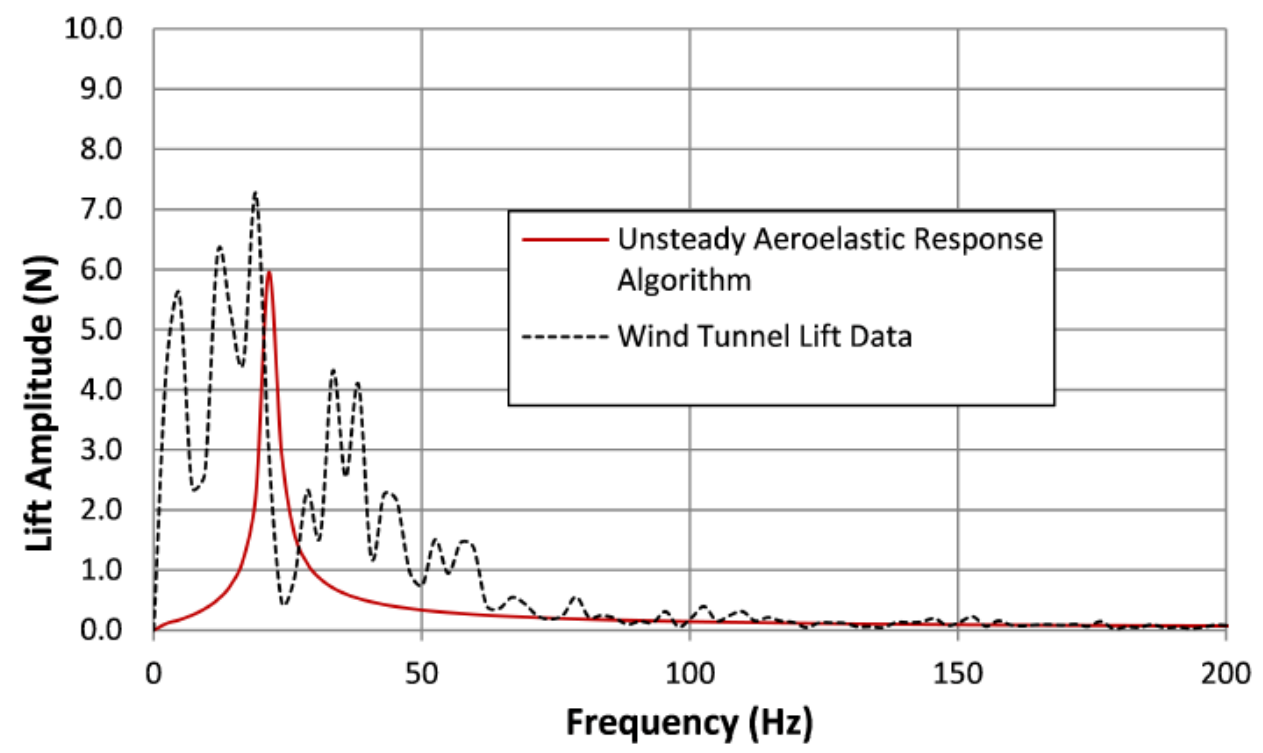

Figure 3.24: Lift results at $-10 \mathrm{deg}$ angle of attack and $V_{0}=17.3 \frac{\mathrm{m}}{\mathrm{s}}$ [2]

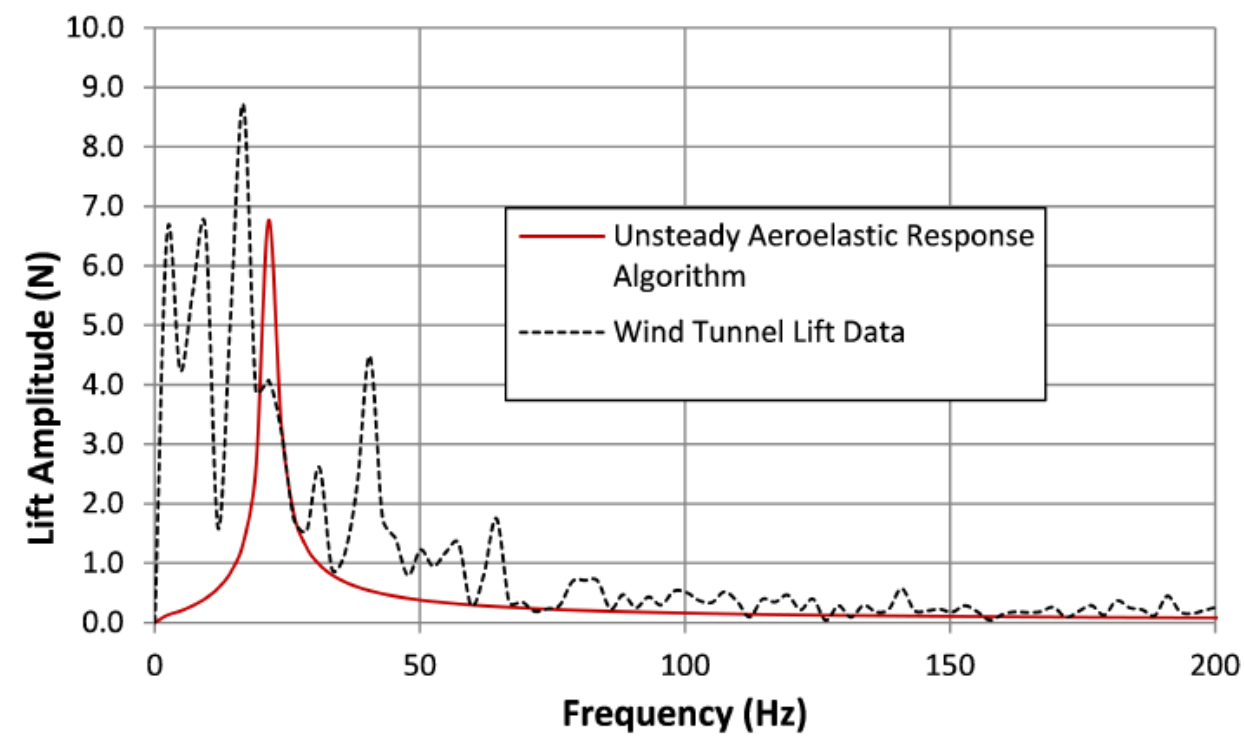

Figure 3.25: Lift results at -10 deg angle of attack and $V_{0}=19.7 \frac{\mathrm{m}}{\mathrm{s}}$ [2] 


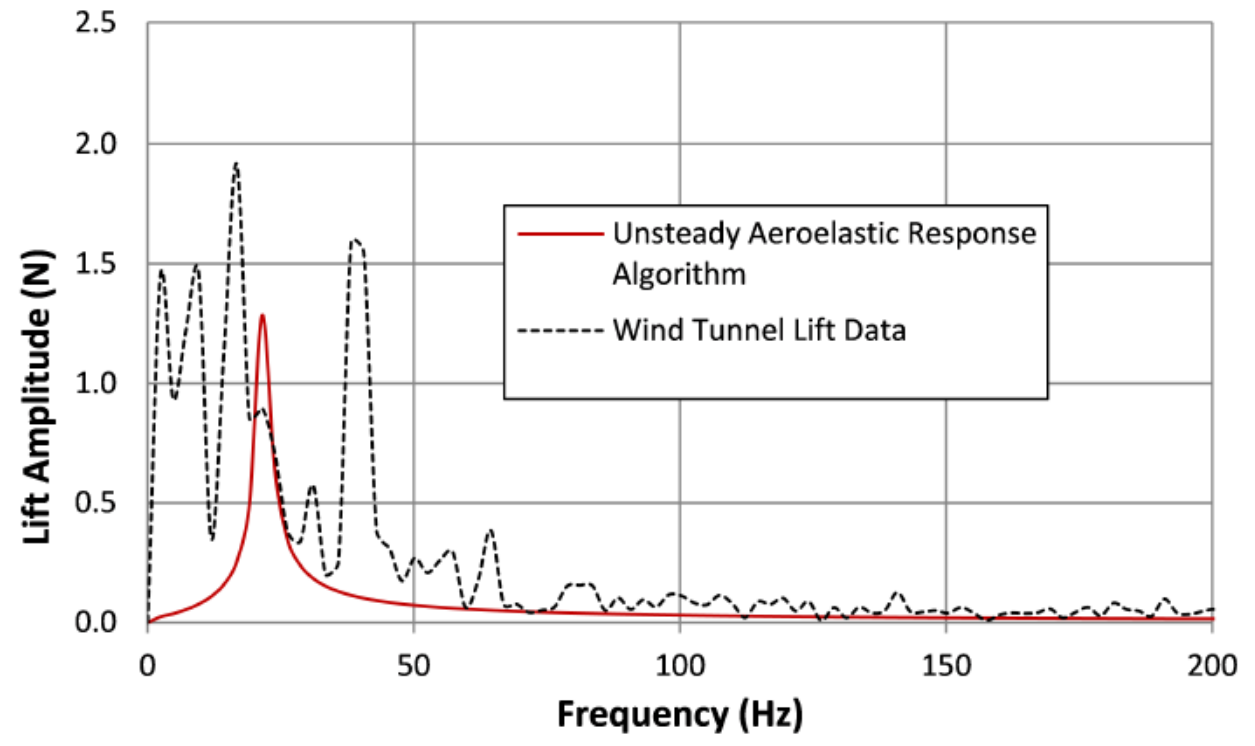

Figure 3.26: Lift results at 0 deg angle of attack and $V_{0}=17.3 \frac{\mathrm{m}}{\mathrm{s}}$ [2]

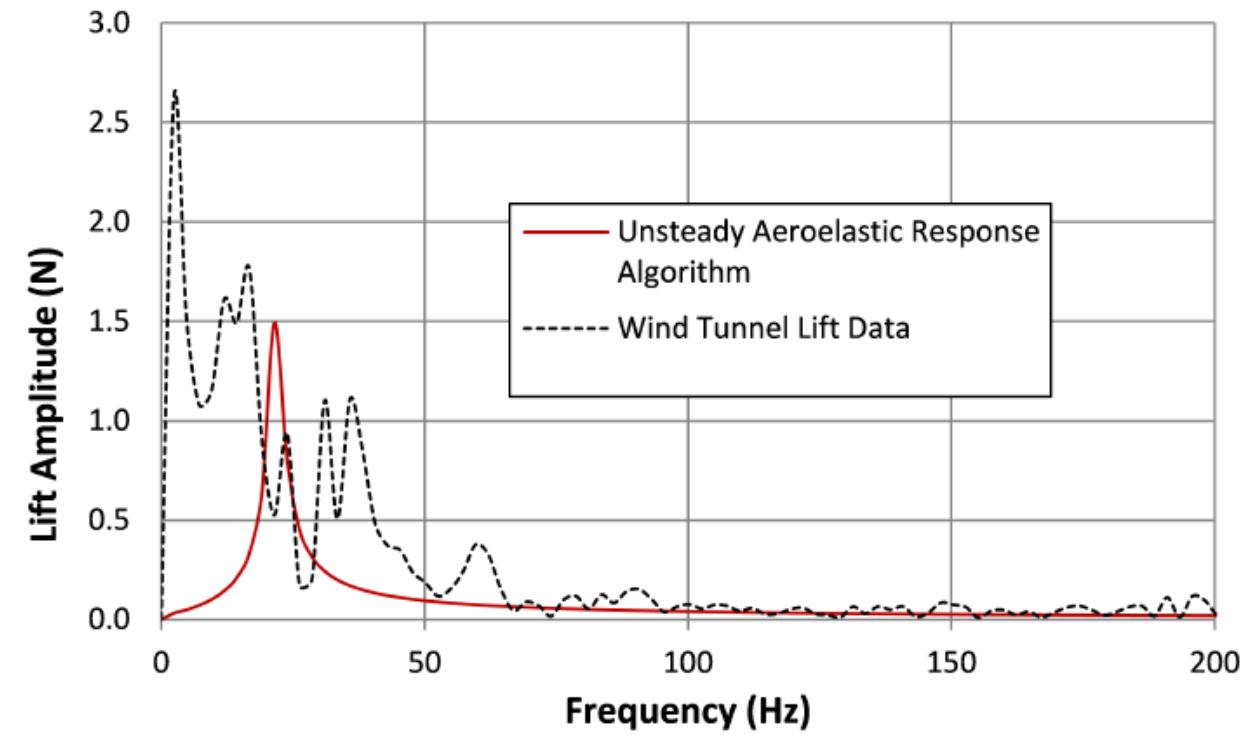

Figure 3.27: Lift results at 0 deg angle of attack and $V_{0}=19.7 \frac{\mathrm{m}}{\mathrm{s}}$ [2] 


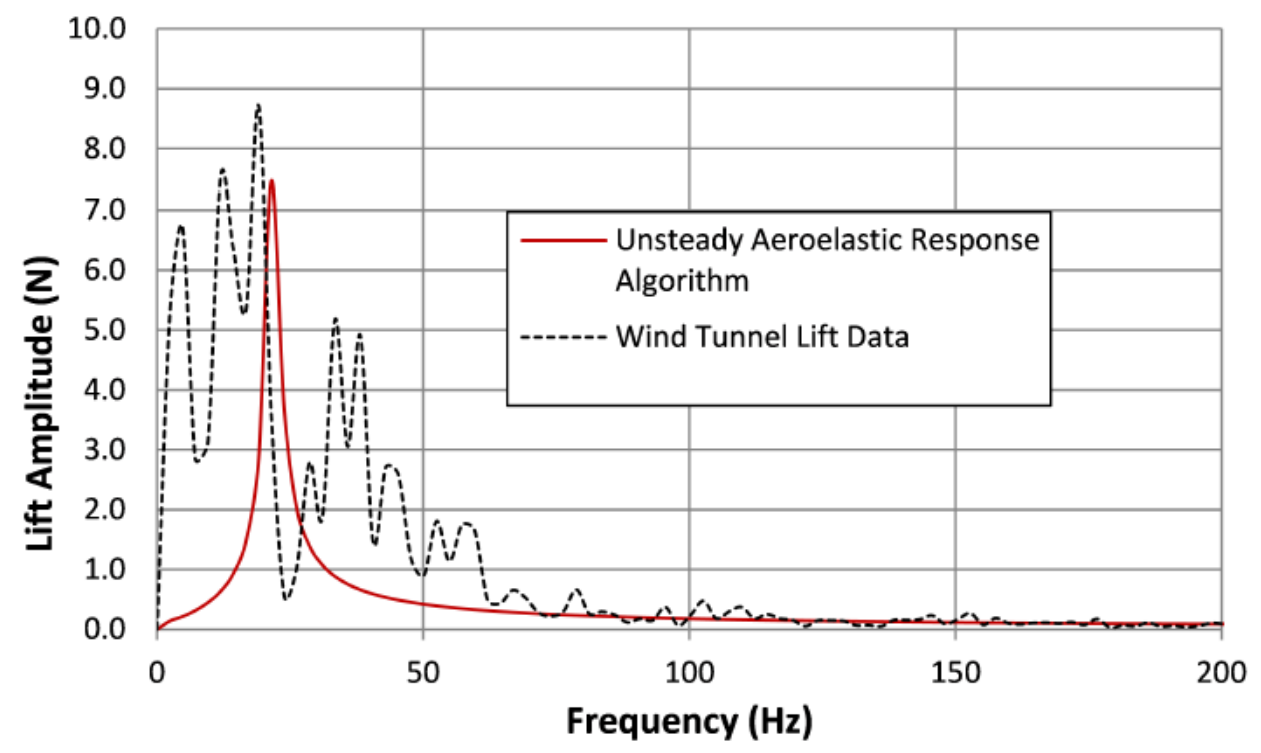

Figure 3.28: Lift results at $10 \mathrm{deg}$ angle of attack and $V_{0}=17.3 \frac{\mathrm{m}}{\mathrm{s}}$ [2].

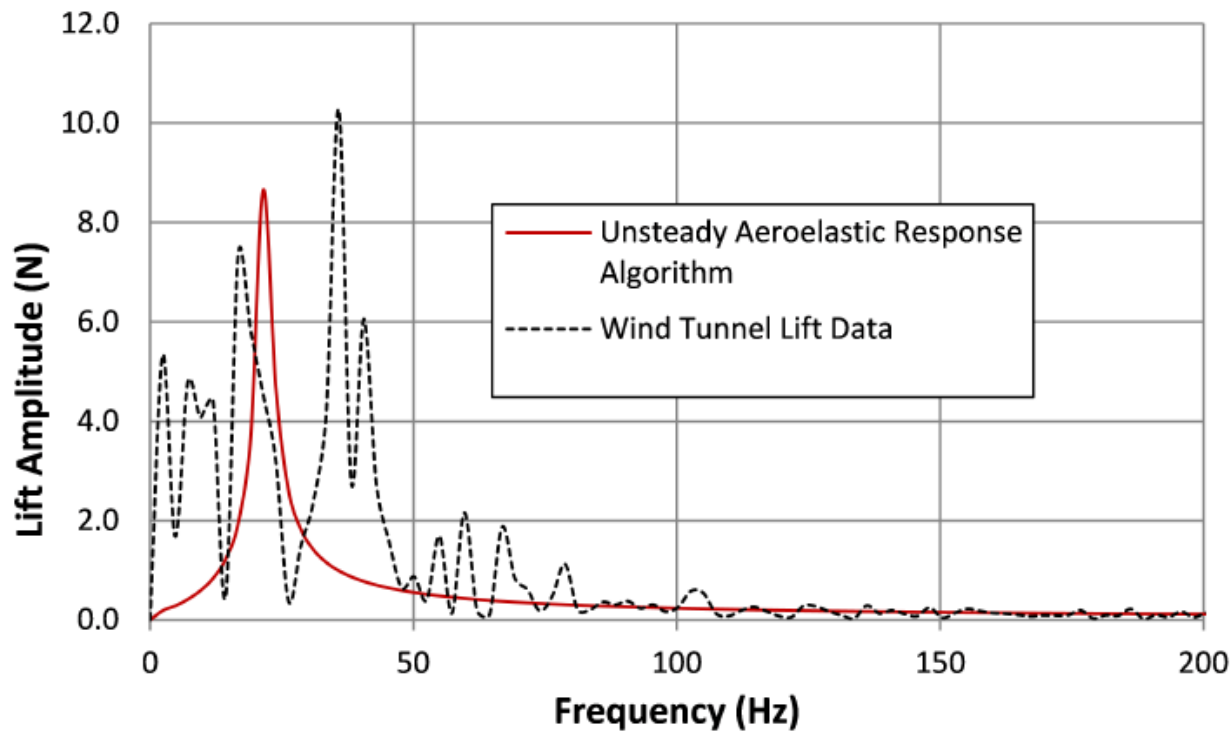

Figure 3.29: Lift results at $10 \mathrm{deg}$ angle of attack and $V_{0}=19.7 \frac{\mathrm{m}}{\mathrm{s}}$ [2]. 


\section{Chapter 4}

\section{CFD and FEA Analysis}

The next step was to obtain validation data through the use of CFD and FEA analyses using the ANSYS package. The CFD analysis was carried out to confirm that the developed PAAC-WTB algorithm [2] generates accurate and correct lift coefficient $C_{L}$ values for $2 \mathrm{D}$ airfoils and rotor blades. 2D and 3D CFD simulations were performed using the ANSYS Fluent package on the S809 airfoil and S809 reduced-scale model and compared against results produced by the PAAC-WTB algorithm and established S809 experimental data. Aeroelastic analysis, which couples CFD and FEA analysis, was then performed on the S809 blade in ANSYS, and compared to results obtained from the algorithm.

\subsection{S809 Two-dimensional CFD Analysis}

For the 2D portion of the CFD analysis, two types of simulations were performed:

1. Static airfoil simulation with no predefined airfoil motion; and

2. Dynamic airfoil simulation with predefined airfoil motion using user defined functions (UDF). 


\subsubsection{Static 2D CFD Analysis}

The purpose of the static 2D CFD analysis was to validate Fluent's accuracy for determining the S809 airfoil's lift coefficient in comparison to experimental data. Meshing of the airfoil was performed utilizing the ICEM CFD meshing software from the ANSYS package. Two meshes were considered for the static airfoil simulations where the airfoil is affixed at several angles of attack and the lift coefficient $C_{L}$ is determined at each respective angle. The first mesh is an unstructured square grid depicted in Figure 4.1.

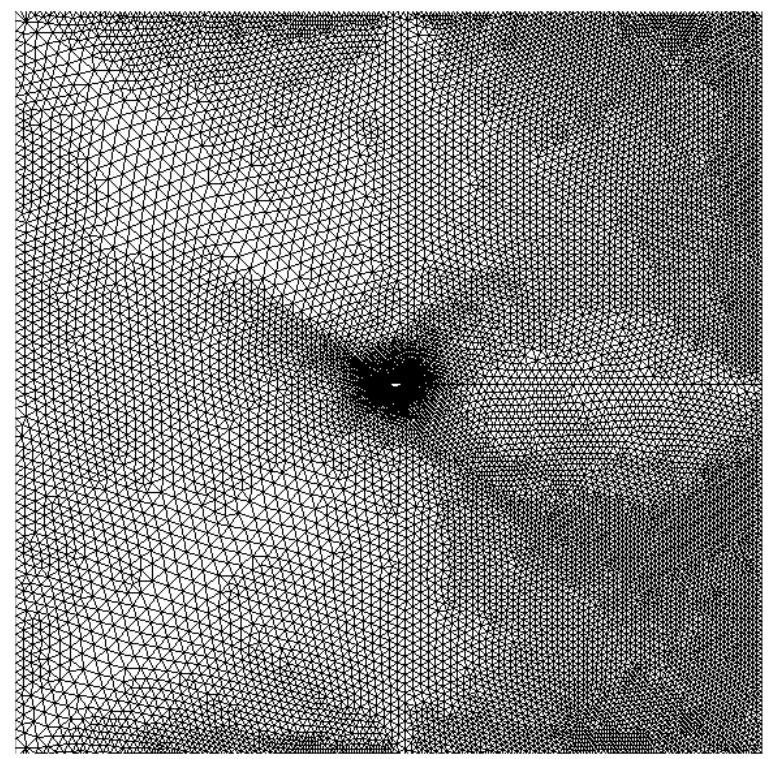

Figure 4.1: S809 airfoil square grid unstructured mesh.

This mesh is composed of purely triangular quadratic elements with six nodes per element, and two degrees of freedom per node, which allows for a simpler meshing approach but less control on the mesh cell count. High cell density is set around the airfoil, including the leading and trailing edges, with a more coarse mesh moving away 
from the airfoil. This refinement in the mesh, shown in Figure 4.2, allowed for more accurate lift coefficient $C_{L}$ results while simultaneously reducing simulation runtime.

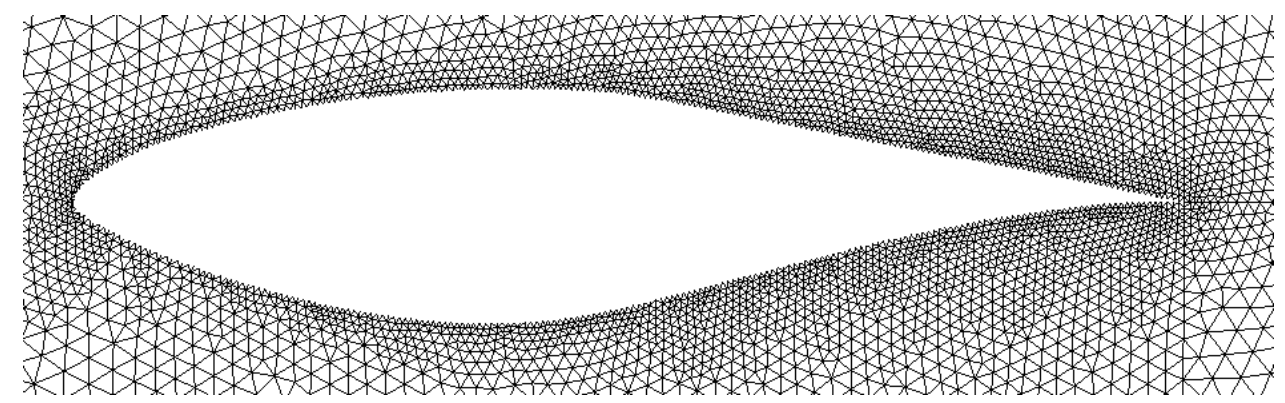

Figure 4.2: Unstructured refined mesh around S809 airfoil.

The unstructured mesh computational domain extends 20 times the airfoil chord length in all four directions around the airfoil, as indicated in Figure 4.3. This is done to accurately simulate far-field conditions with a fully-developed velocity profile at the airfoil.

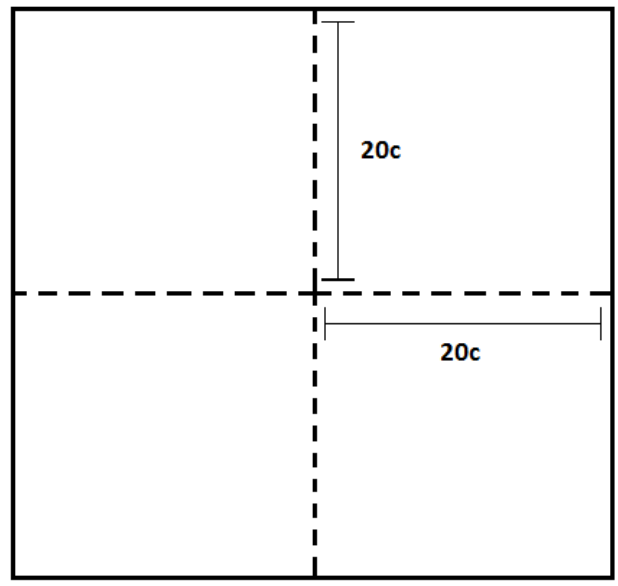

Figure 4.3: Unstructured mesh computational domain.

The second mesh considered is a structured C-grid mesh depicted in Figure 4.4. 


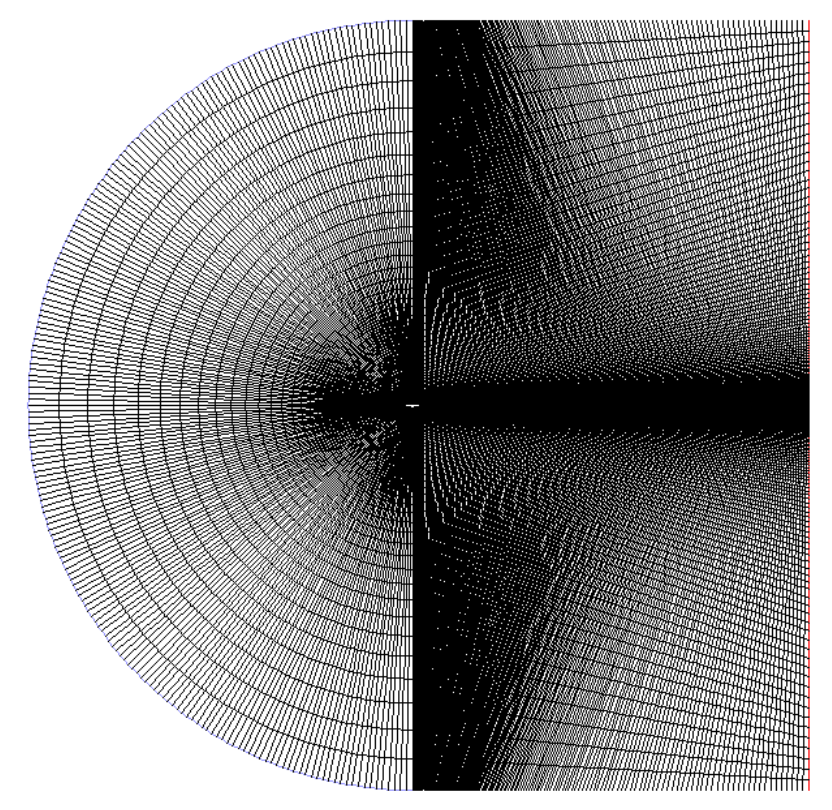

Figure 4.4: S809 airfoil C-grid structured mesh.

This mesh is composed of purely quadrilateral elements, efficiently spaced in a closelypacked arrangement. This allows for a higher degree of control over cell count and arrangement. The meshing approach is similar to the unstructured mesh, with a high mesh density around the airfoil achieved by defining more quadrilateral grids around the airfoil, as shown in Figure 4.5.

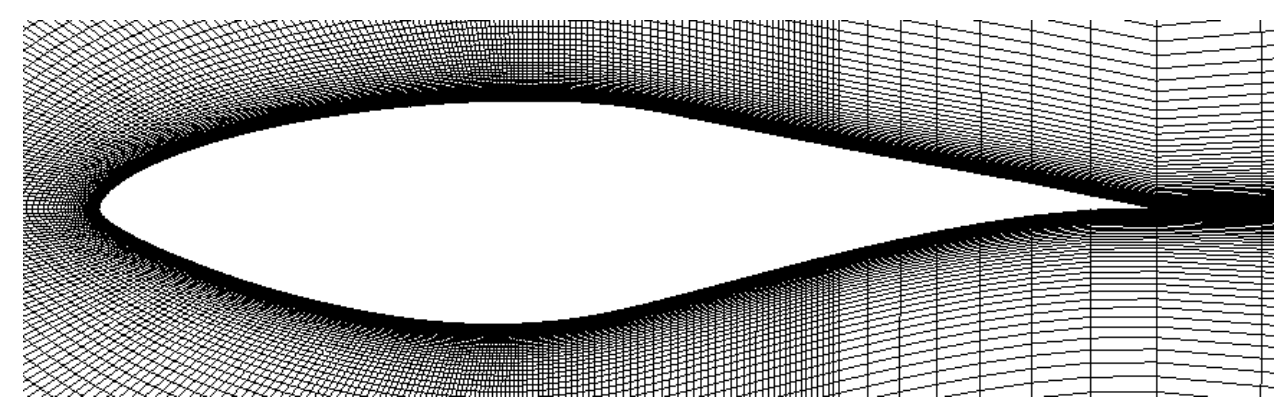

Figure 4.5: Structured refined mesh around the S809 airfoil. 
The structured mesh computational domain was set 20 times chord length downstream and upstream of the airfoil and 20 times chord length in width, as indicated in Figure 4.6.

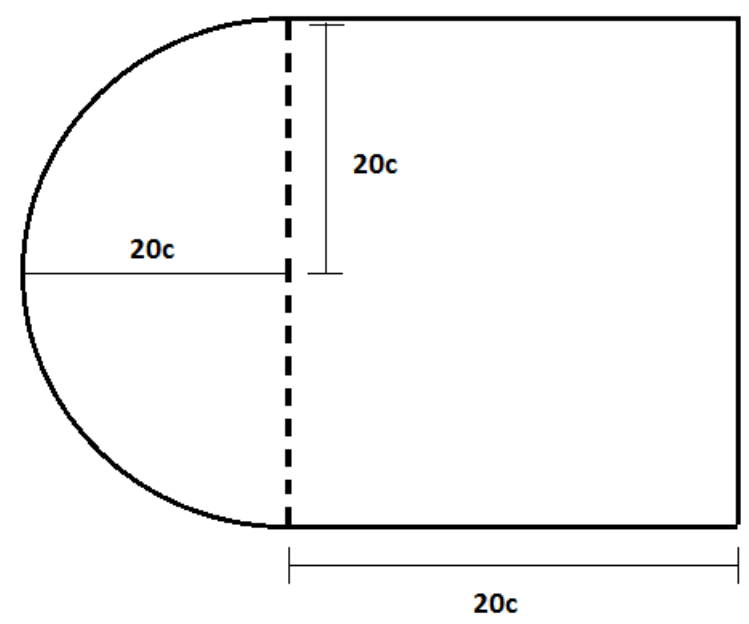

Figure 4.6: Structured mesh computational domain ( $c$ indicates airfoil chord length).

For the static airfoil simulations, both meshes were used to determine the lift coefficient $C_{L}$ at different angles of attack and were compared against experimental data obtained from the NREL Delft University wind tunnel experiment [10]. The experimental parameters for the simulations performed are listed in Table 4.1.

Data used from the Delft University wind tunnel experiment was limited to the first test performed at $R e=1 \times 10^{6}$. The S809 airfoil used in the experiment had a chord length of $0.61 \mathrm{~m}$. The airfoil used for the CFD simulations has a chord length of $1 \mathrm{~m}$. Thus in order to achieve the same Reynolds number used in the experiment, the velocity of the CFD simulation had to be matched using Reynold's equation

$$
R e=\frac{\rho v c}{\mu}
$$


Table 4.1: 2D simulation parameters.

\begin{tabular}{|c|c|}
\hline Parameter & Value \\
\hline Fluid & Air \\
\hline Dynamic viscosity $\mu$ & $1.7894 \times 10^{5} \frac{\mathrm{kg}}{\mathrm{m}-\mathrm{s}}$ \\
\hline Density $\rho$ & $1.225 \frac{\mathrm{kg}}{\mathrm{m}^{3}}$ \\
\hline Pressure & $101325 \mathrm{~Pa}$ \\
\hline Reynolds number $R e$ & $1 \times 10^{6}$ \\
\hline Simulation settings & Steady state and k-omega turbulence model \\
\hline Boundary conditions & Pressure far field and no slip walls \\
\hline Wind speed & $14.65 \frac{\mathrm{m}}{\mathrm{s}}$ \\
\hline
\end{tabular}

where $c$ is the chord length of the airfoil. Thus to achieve an equivalent Reynold's number for the CFD analysis, an incoming constant wind speed of $14.65 \mathrm{~m} / \mathrm{s}$ is required. To obtain data at each angle of attack for the unstructured mesh, the airfoil pitch was varied using UDF. This is feasible due to the triangular elements used in the mesh which accommodate mesh deformations. For the structured mesh, however, the grid's quadrilateral elements face an issue with mesh deformation when pitching the airfoil, particularly at higher angles of attack. Thus, the incoming wind velocity vector at the inlet is modified instead for each angle of attack.

Lift coefficient data from the experiment was compared against data obtained from the structured and unstructured meshes. Results are shown in Table 4.2.

The structured and unstructured mesh results show good agreement to the wind tunnel experimental results, as shown in Figure 4.7. Both mesh types were deemed suitable for further analyses. During computation it was noted that the lift coefficient data oscillated throughout most of the computational run time before converging. This can be attributed to the consideration of turbulence modelling, in particular the vortex shedding effect at the tail. The structured computation also experienced a 
Table 4.2: Lift coefficient $C_{L}$ data comparison.

\begin{tabular}{|c|c|c|c|}
\hline Angle (degrees) & Experimental $C_{L}$ & Structured mesh $C_{L}$ & Unstructured mesh $C_{L}$ \\
\hline 0.0 & 0.139 & 0.117 & 0.0985 \\
\hline 2.1 & 0.378 & 0.353 & 0.318 \\
\hline 4.1 & 0.617 & 0.645 & 0.662 \\
\hline 6.16 & 0.851 & 0.812 & 0.872 \\
\hline 8.2 & 0.952 & 0.964 & 0.973 \\
\hline 10.21 & 0.952 & 1.034 & 1.184 \\
\hline 11.21 & 0.947 & 1.0768 & 1.062 \\
\hline
\end{tabular}

higher degree of lift coefficient oscillations in comparison to the unstructured mesh computation and a higher number of iterations for convergence. This might be due to the method by which the angle of attack is adjusted for the structured mesh. The $\mathrm{x}$ and y components of the inlet flow velocity are adjusted instead of pitching the airfoil to the desired angle. This results in the incoming flow to not align with the airfoil's denser mesh region at higher angles of attack [24]. However, the effect is negligible at the observed angles of attack and is more prominent at much higher angles (20 deg). 


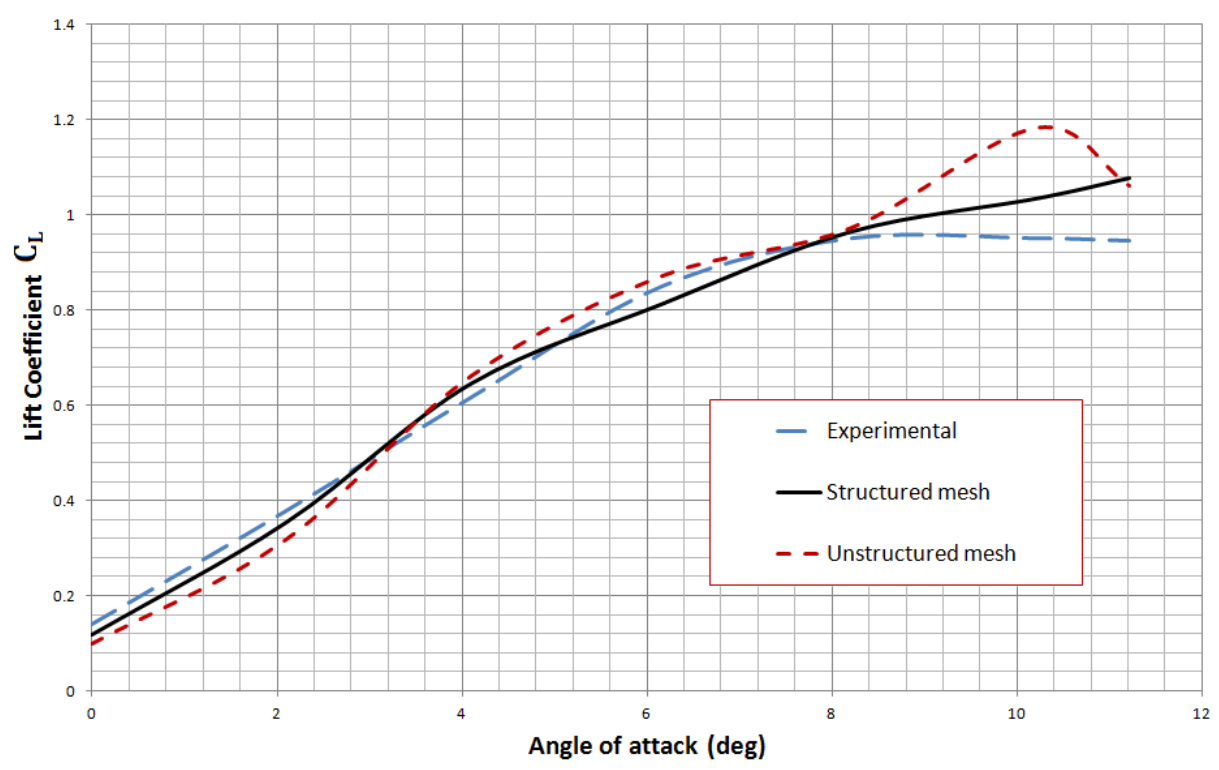

Figure 4.7: Lift coefficient data comparison

\subsubsection{Dynamic 2D CFD analysis}

The purpose of the dynamic analyses is to validate the 2D Panel Method algorithm portion of the PAAC-WTB algorithm. The unstructured grid was used for this set of analyses. Since the mesh consists of purely triangular elements, it allows for deformation and realignment of the mesh elements around the airfoil during its motion. Although the use of a hybrid quadrilateral-triangular element mesh is slightly more preferable for airfoil analysis, due to mesh deformation limitations, a purely triangular mesh was used instead.

Impulse, Theordorsen, and Wagner functions were used to define both harmonic and non-harmonic pitching, plunging, and a combination of pitching and plunging motions of the airfoil, as depicted in Figure 4.8. For all analyses performed, user defined functions (UDF) were written in $\mathrm{C}$ code, compiled, and assigned to the mesh to define the airfoil motion. A sample UDF code is available in Appendix A. It is important to note that all CFD analyses performed assume laminar flow with no turbulence modelling, with the exception of the static 2D CFD analyses performed 


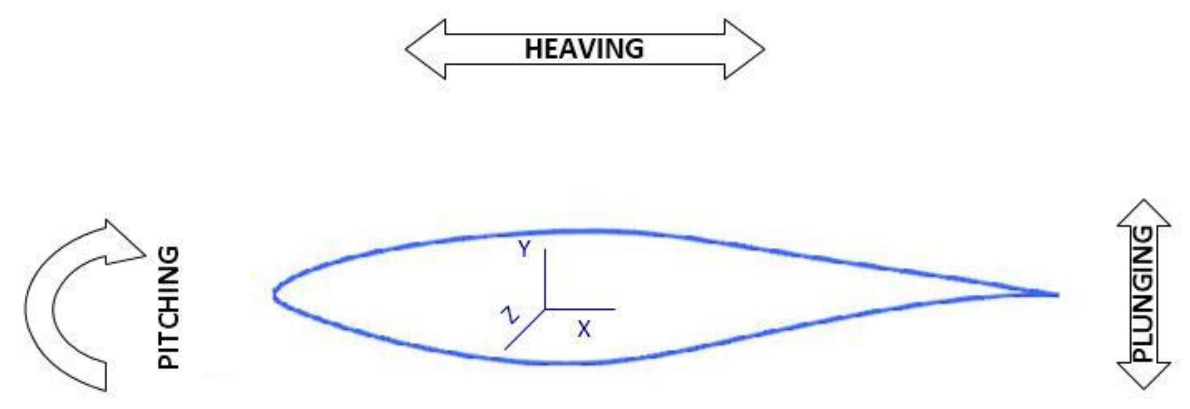

Figure 4.8: The three primary airfoil motions.

in Section 4.1.1. All 2D dynamic CFD analyses assume pressure far-field conditions and a no-slip boundary condition at the surface of the airfoil.

The first set of analyses dealt with general non-harmonic impulse functions. The centre of gravity about which the UDFs are defined is at the leading edge, point $(0,0)$. The UDFs are defined using the velocity functions derived from the displacement functions. With reference to Figure 4.9, the pitching motion is defined by:

$$
\theta(t)=-0.2 \exp ^{-10(t-1.0)^{2}} \sin (\pi t)
$$

and

$$
\dot{\theta}(t)=4.0 \exp ^{-10(t-1.0)^{2}}(t-1.0) \sin (\pi t)-0.2(\pi) \exp -10(t-1.0)^{2} \cos (\pi t)
$$

while the plunging motion is defined by:

$$
h(t)=-0.05 \exp ^{-20(t-1.25)^{2}}
$$


and

$$
\dot{h}(t)=2.0 \exp ^{-20(t-1.25)^{2}}(t-1.25)
$$

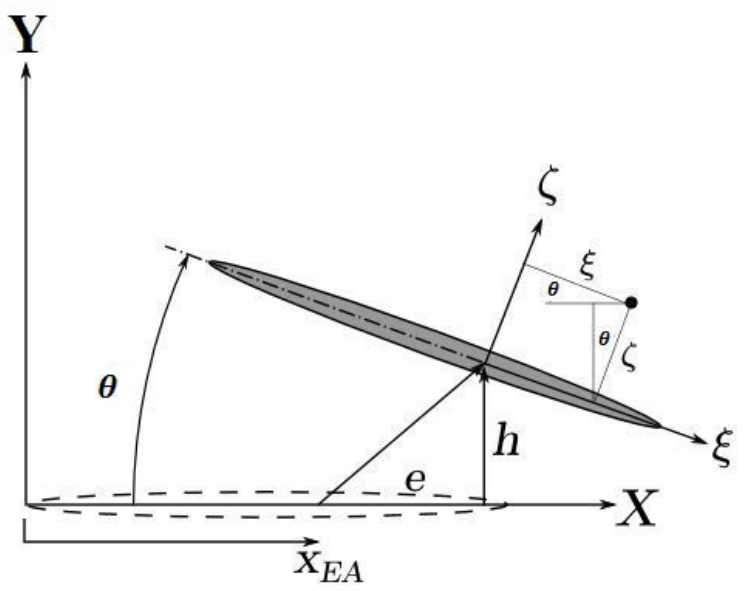

Figure 4.9: Airfoil motion variables [25].

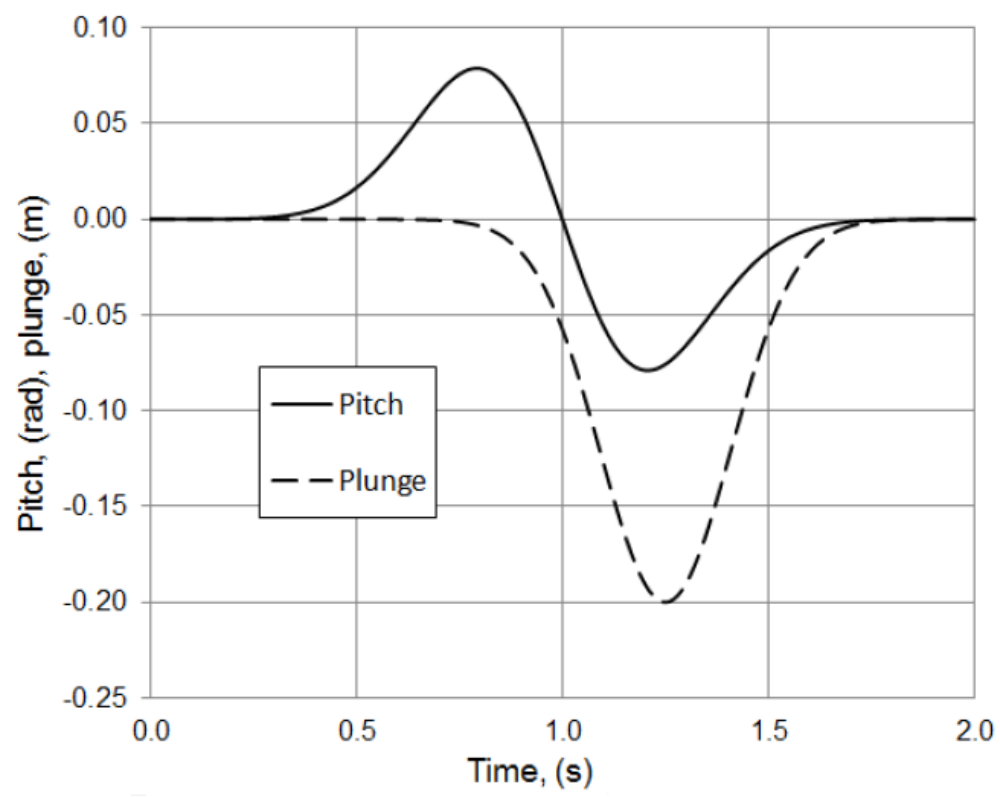

Figure 4.10: Plot of non-harmonic motion functions [25]. 
Table 4.3: CFD simulation parameters for impulse functions.

\begin{tabular}{|c|c|}
\hline Parameter & Value \\
\hline Simulation run 1 & Pitching only, Equation 4.3 \\
\hline Simulation run 2 & Plunging only, Equation 4.5 \\
\hline Simulation run 3 & Pitching and plunging, Equations 4.3 and 4.5 \\
\hline Freestream velocity $V_{0}$ & $20 \frac{\mathrm{m}}{\mathrm{s}}$ \\
\hline Density & $1.225 \frac{\mathrm{kg}}{\mathrm{m}^{3}}$ \\
\hline Airfoil Chord & $1 \mathrm{~m}$ \\
\hline Simulation end time & $2 \mathrm{~s}$ \\
\hline Number of timesteps, $t_{k}$ & 200 \\
\hline Number of elements & 34,902 \\
\hline
\end{tabular}

where $\theta$ and $h$ are the pitch and plunge coordinates, respectively. The motions depicted by the equations are plotted in Figure 4.10.

Three simulation runs were performed using the above functions, with the same simulation parameters but a different UDF for each desired airfoil motion. Table 4.3 shows the simulation parameters. Lift coefficient results obtained were then compared to the results obtained from the 2D Unsteady Panel Method used in the PAAC-WTB algorithm [2].

Plots of the lift coefficient $C_{L}$ data are shown in Figures 4.11, 4.12, and 4.13. The data shows reasonable agreement between the Fluent results and the PAACWTB algorithm. Discrepancies can be attributed to the mesh quality, differences in the flow's velocity profile development over time, and inherent differences between Fluent's pressure-based solver and the 2D Panel Method. 


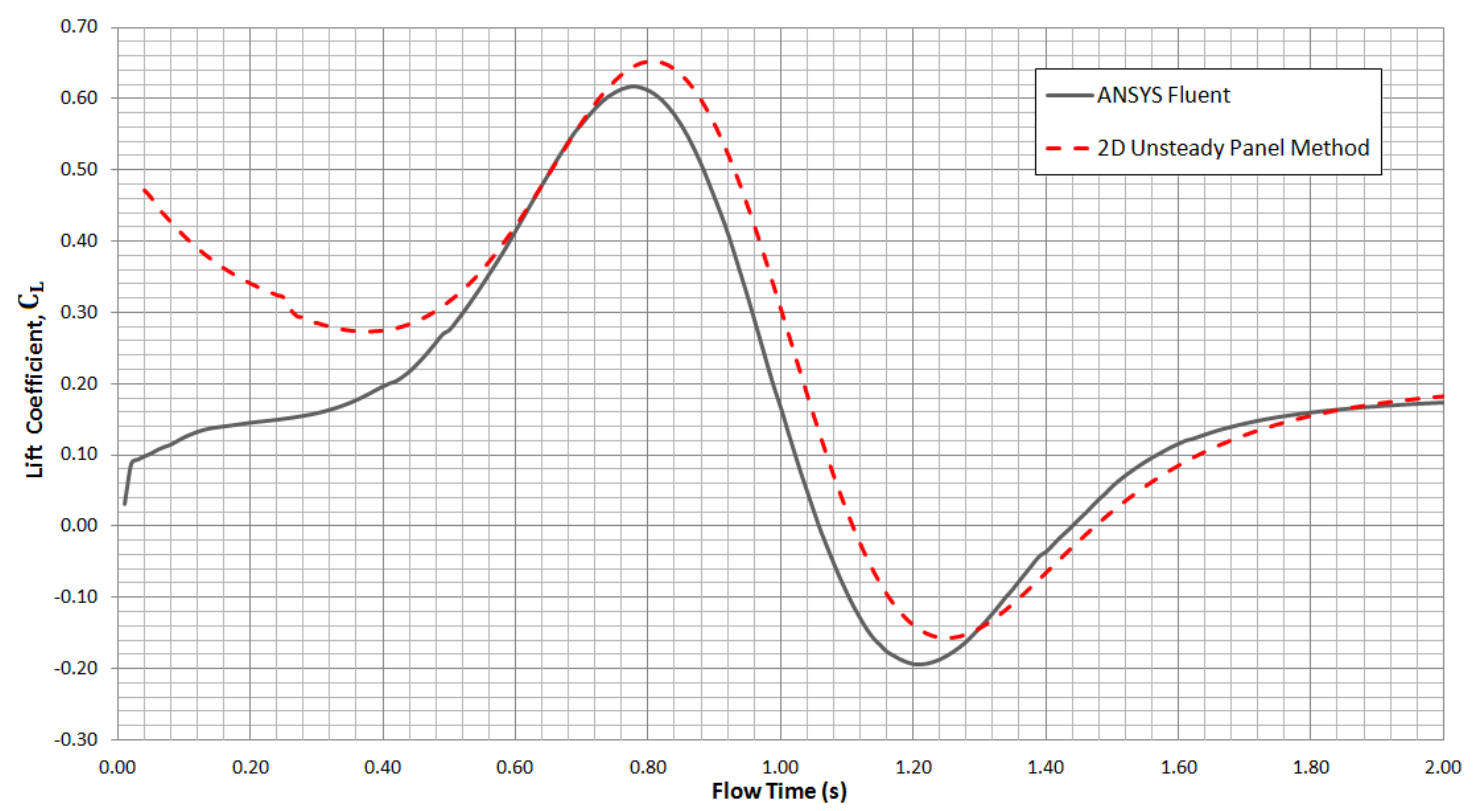

Figure 4.11: Pitching only lift coefficient results.

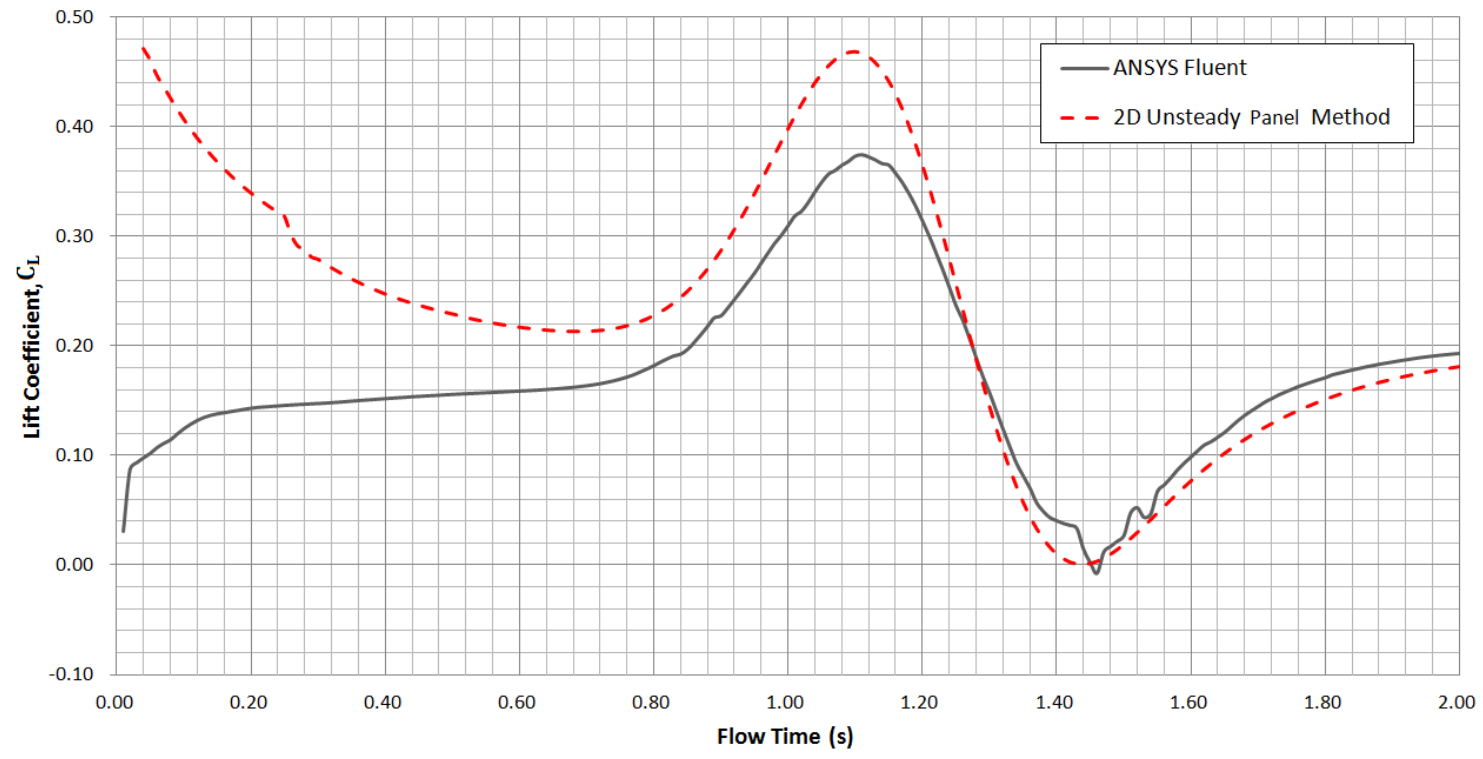

Figure 4.12: Plunging only lift coefficient results. 


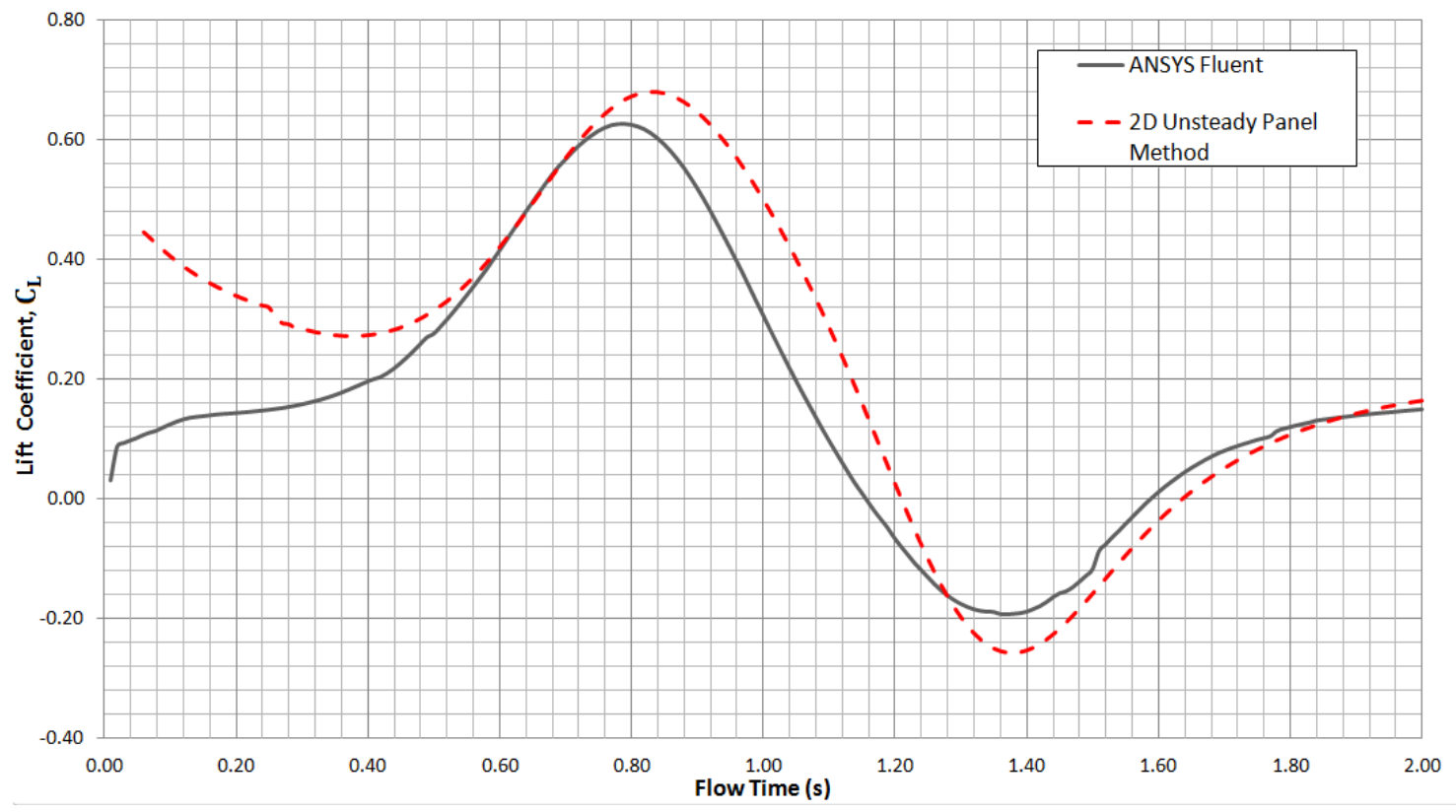

Figure 4.13: Pitching and plunging lift coefficient results.

\subsubsection{Theodorsen's Solution for Harmonic Airfoil Motion}

The second set of analyses were motions that were based on Theodorsen's unsteady model $[2,26]$. The purpose of Theodorsen's time-dependent solution is to provide a magnitude and phase change to the aerodynamic loading, that is harmonic in nature $[25,27]$. Theordorsen's derived the following equations which provide the lift and moment on an airfoil experiencing harmonic pitching and plunging motions, where $\mathrm{x}$ and $\mathrm{n}$ are airfoil geometric parameters shown in Figure 4.14.

$$
L=\frac{1}{2} c_{l_{\alpha}} \rho n^{2}\left[\ddot{h}+V_{0} \dot{\theta}-n x \ddot{\theta}\right]+\frac{1}{2} c_{l_{\alpha}} \rho n C(k)\left[V_{0} \dot{h}+V_{0}^{2} \theta+V_{0} n\left(\frac{1}{2}-x\right) \dot{\theta}\right]
$$




$$
\begin{gathered}
M=\frac{1}{2} c_{l_{\alpha}} \rho n^{2}\left[n x \ddot{h}-V_{0} n\left(\frac{1}{2}-x\right) \dot{\theta}-n^{2}\left(\frac{1}{8}+x\right) \ddot{\theta}\right]+ \\
\frac{1}{2} c_{l_{\alpha}} \rho n^{2}\left(x+\frac{1}{2}\right) C(k)\left[V_{0} \dot{h}+V_{0}^{2} \theta+V_{0} n\left(\frac{1}{2}-x\right) \dot{\theta}\right],
\end{gathered}
$$

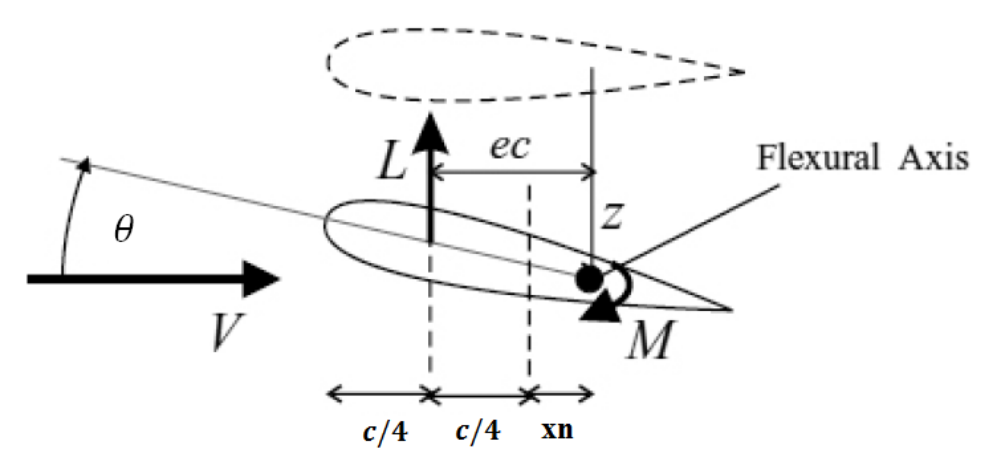

Figure 4.14: 2D airfoil geometry, reproduced from [2, 27].

Several simulations were performed with motions of pitching, plunging, and a combination of pitching and plunging at different reduced frequencies $k$. The reduced frequency is an aeroelastic parameter that indicates the degree of unsteadiness the airfoil experiences [28]. It is calculated as:

$$
k=\frac{\omega c}{2 V_{0}}
$$

\subsubsection{Pitching Motion}

A set of simulation trials was performed for a harmonic pitching-only UDF assigned to the airfoil. Simulation parameters are shown in Table 4.4. The pitching motion of the airfoil is given by, 
Table 4.4: CFD simulation parameters for pitching-only motion.

\begin{tabular}{|c|c|}
\hline Parmater & Value \\
\hline Simulation motion & Pitching only, Equation 4.9 \\
\hline Density $\rho$ & $1.225 \frac{\mathrm{kg}}{\mathrm{m}^{3}}$ \\
\hline Airfoil chord $c$ & $1 \mathrm{~m}$ \\
\hline Simulation end time & $4 \mathrm{~s}$ \\
\hline Number of timesteps $t_{k}$ & 200 \\
\hline Number of elements & 34,902 \\
\hline Trial 1 & $\omega=12 \frac{\mathrm{rad}}{\mathrm{s}}, \mathrm{k}=0.20, \mathrm{~V}_{0}=30 \frac{\mathrm{m}}{\mathrm{s}}$ \\
\hline Trial 2 & $\omega=12 \frac{\mathrm{rad}}{\mathrm{s}}, \mathrm{k}=0.50, \mathrm{~V}_{0}=12 \frac{\mathrm{m}}{\mathrm{s}}$ \\
\hline Trial 3 & $\omega=6 \frac{\mathrm{rad}}{\mathrm{s}}, \mathrm{k}=1.0, \mathrm{~V}_{0}=6 \frac{\mathrm{m}}{\mathrm{s}}$ \\
\hline
\end{tabular}

$$
\dot{\theta}(t)=A_{\text {rot }} \omega_{\text {pitch }} \cos \left(\dot{\varphi}\left(t+\frac{\pi}{2}\right)\right)
$$

where $A_{r o t}$ is the pitching rotational amplitude, $\omega_{\text {pitch }}$ is the angular frequency, and $t$ is the simulation runtime.

Lift coefficient results from the CFD simulations were compared to the 2D Panel Method results from the PAAC-WTB algorithm results and Theodorsen's unsteady model solution shown in Figures 4.15, 4.16, and 4.17. The results show minor discrepancies between the ANSYS Fluent results and the 2D Panel Method results. This may be due to the mesh quality as well as the difference between the pressure-based solver Fluent utilizes and the 2D Panel Method the PAAC-WTB algorithm utilizes. 


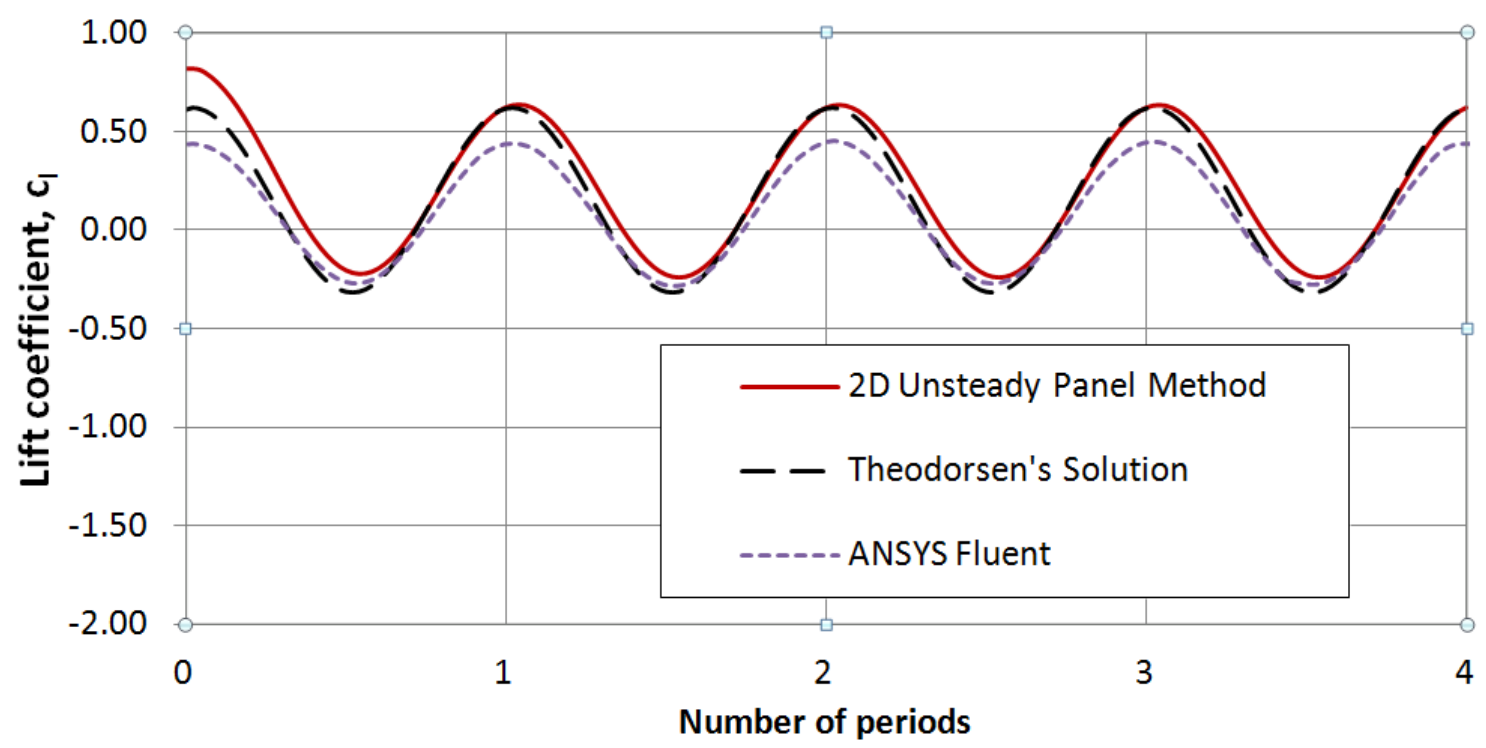

Figure 4.15: Comparison of lift coefficient data for pitching-only case, $k=0.20$, $\omega=12 \frac{\mathrm{rad}}{\mathrm{s}}$, and $V_{0}=30 \frac{\mathrm{m}}{\mathrm{s}}[2]$.

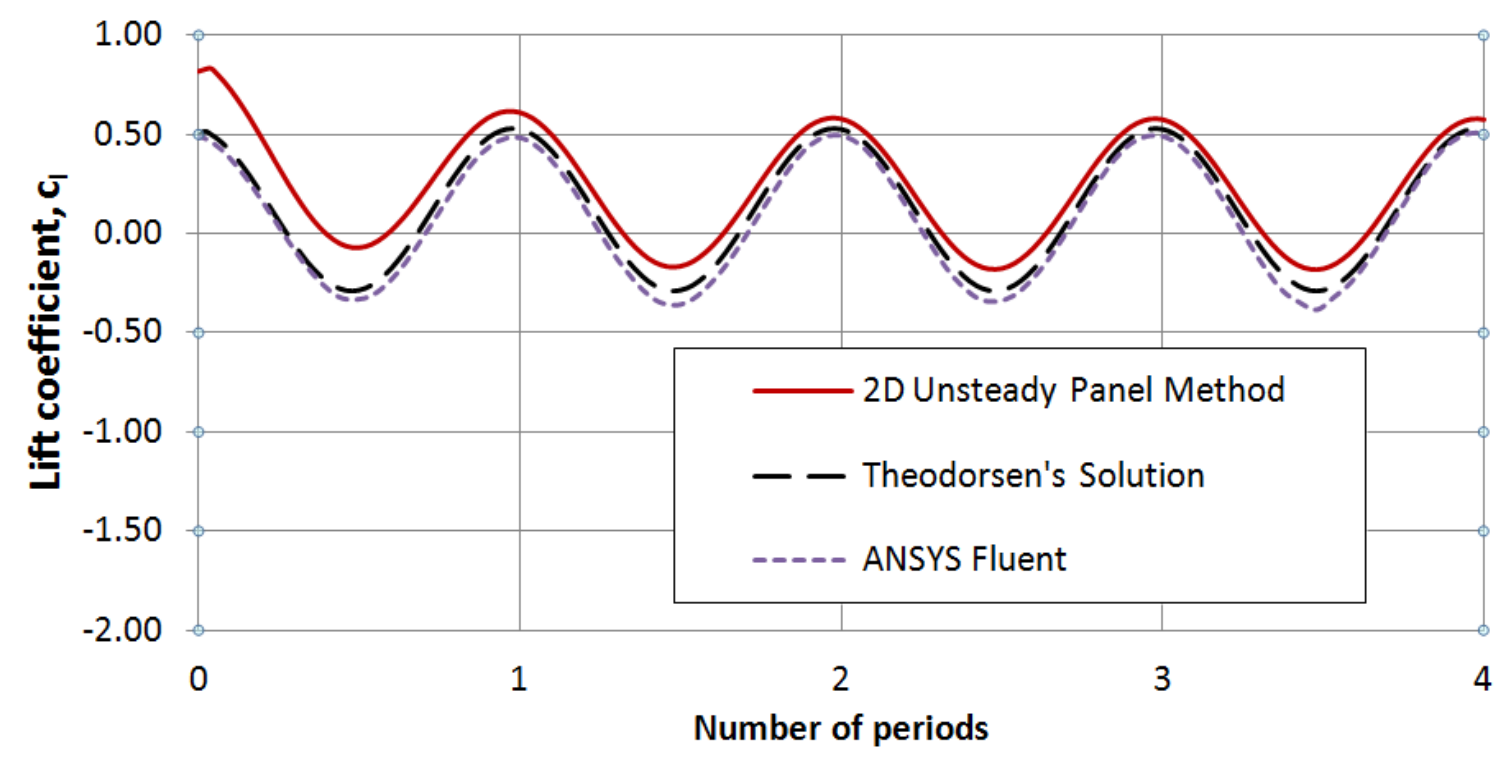

Figure 4.16: Comparison of lift coefficient data for pitching-only case, $k=0.50$, $\omega=12 \frac{\mathrm{rad}}{\mathrm{s}}$, and $V_{0}=12 \frac{\mathrm{m}}{\mathrm{s}}[2]$. 


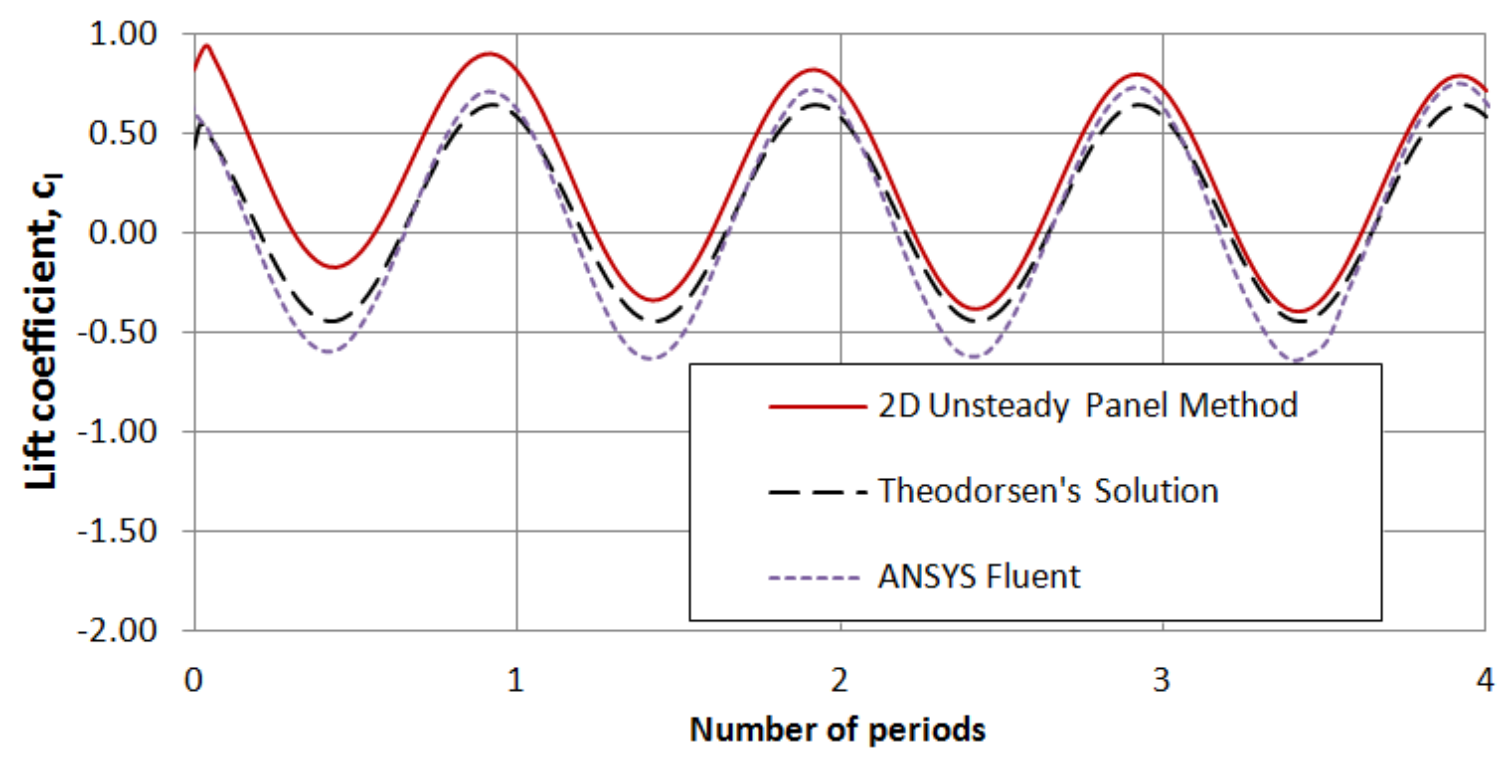

Figure 4.17: Comparison of lift coefficient data for pitching-only case, $k=1.0$, $\omega=6 \frac{\mathrm{rad}}{\mathrm{s}}$, and $V_{0}=6 \frac{\mathrm{m}}{\mathrm{s}}[2]$.

\subsubsection{Pitching and Plunging Motion}

Another set of simulations was performed for a harmonic pitching and plunging motion UDF assigned to the airfoil. Equations 4.9 and 4.10 show the pitching and plunging functions respectively. The simulation parameters are shown in Table 4.5.

$$
\dot{h}(t)=A_{\text {plunge }} \omega_{\text {plunge }} \cos \left(\omega_{\text {plunge }}\left(t+\frac{\pi}{2}\right)\right)
$$

where $A_{\text {plunge }}$ is the plunging amplitude, $\omega_{\text {plunge }}$ is the angular frequency, and $t$ is the simulation runtime. Figures 4.18, 4.19, and 4.20 show good agreement between ANSYS Fluent results and the 2D Panel Method results. Similar to the pitching trials, discrepancies can be attributed to the mesh quality as well as the difference between the pressure-based solver Fluent utilizes and the 2D Panel algorithm. 
Table 4.5: CFD simulation parameters for pitching and plunging motion.

\begin{tabular}{|c|c|}
\hline Parmater & Value \\
\hline Simulation motion & Pitching and plunging, Equations 4.9 and 4.10 \\
\hline Density $\rho$ & $1.225 \frac{\mathrm{kg}}{\mathrm{m}^{3}}$ \\
\hline Airfoil chord $c$ & $1 \mathrm{~m}$ \\
\hline Simulation end time & Trial 1 and $2=2.25 \mathrm{~s}$, Trial $3=1 \mathrm{~s}$ \\
\hline Number of timesteps $t_{k}$ & 200 \\
\hline Number of elements & 34,902 \\
\hline Pitch angle $\alpha$ & $5 \frac{\mathrm{deg}}{\mathrm{s}}$ \\
\hline Wind speed $V_{0}$ & $15 \frac{\mathrm{m}}{\mathrm{s}}, \omega_{\text {plunge }}=12 \frac{\mathrm{rad}}{\mathrm{s}}$ \\
\hline Trial 1 & $k=0.20, \omega_{\text {pitch }}=12 \frac{\mathrm{rad}}{\mathrm{s}}$ \\
\hline Trial 2 & $k=0.50, \omega_{\text {pitch }}=12 \frac{\mathrm{rad}}{\mathrm{s}}, \omega_{\text {plunge }}=30 \frac{\mathrm{rad}}{\mathrm{s}}$ \\
\hline Trial 3 & $k=1.0, \omega_{\text {pitch }}=30 \frac{\mathrm{rad}}{\mathrm{s}}, \omega_{\text {plunge }}=12 \frac{\mathrm{rad}}{\mathrm{s}}$ \\
\hline
\end{tabular}

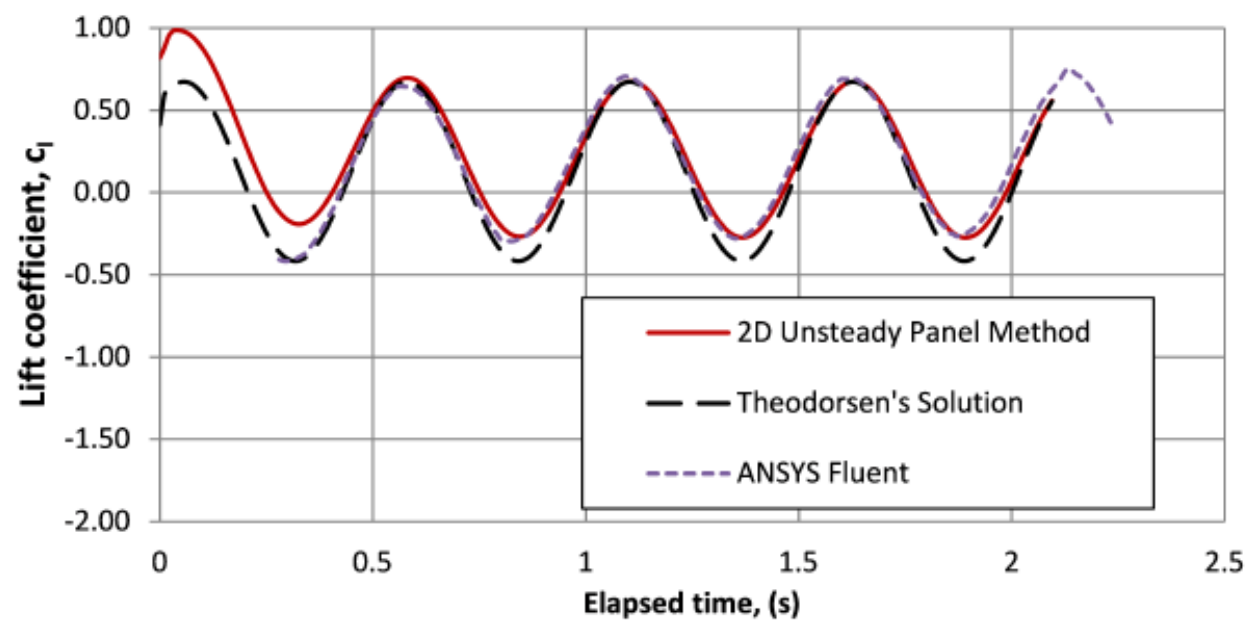

Figure 4.18: Comparison of lift coefficient data pitching and plunging, $k=0.20$, $\omega_{\text {pitch }}=12 \frac{\mathrm{rad}}{\mathrm{s}}$, and $\omega_{\text {plunge }}=12 \frac{\mathrm{rad}}{\mathrm{s}}[2]$. 


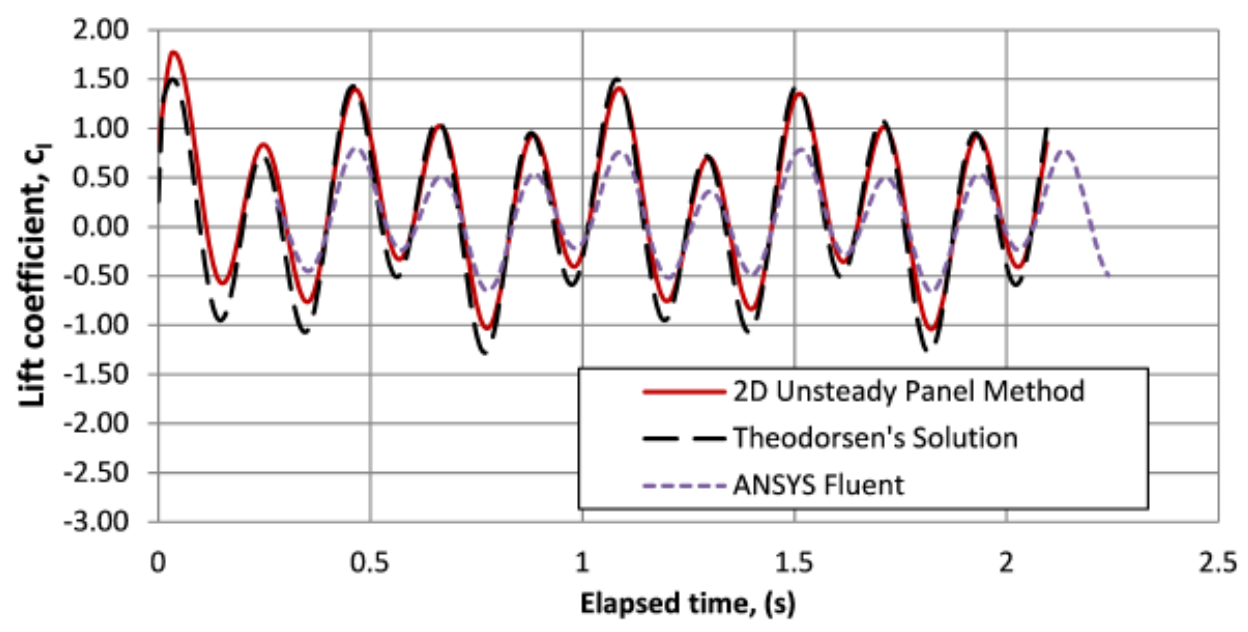

Figure 4.19: Comparison of lift coefficient data pitching and plunging, $k=0.50$, $\omega_{\text {pitch }}=12 \frac{\mathrm{rad}}{\mathrm{s}}$, and $\omega_{\text {plunge }}=30 \frac{\mathrm{rad}}{\mathrm{s}}[2]$.

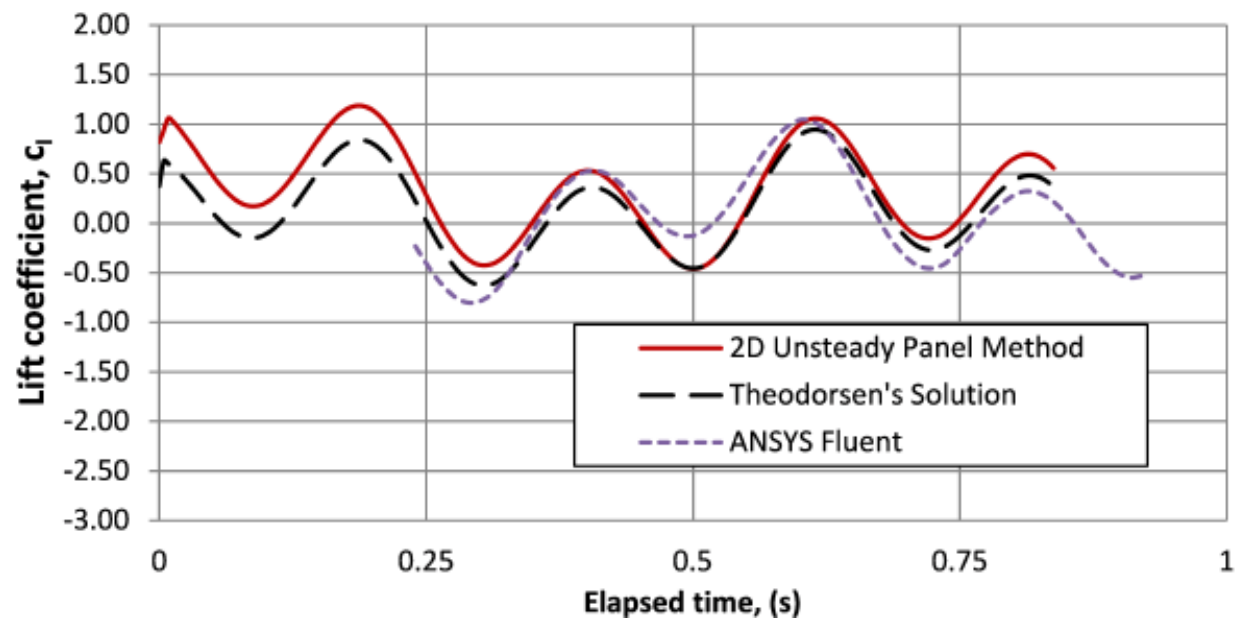

Figure 4.20: Comparison of lift coefficient data pitching and plunging, $k=1.0$, $\omega_{\text {pitch }}=30 \frac{\mathrm{rad}}{\mathrm{s}}$, and $\omega_{\text {plunge }}=12 \frac{\mathrm{rad}}{\mathrm{s}}[2]$. 


\subsubsection{Wagner's Solution Airfoil Motion}

The final set of 2D analyses investigated airfoil motion based on Wagner's solution. Wagner considered the time-dependent lift of an airfoil that experiences a sudden step increase in angle of attack [25, 27]. Two trials were performed with different step changes in the angle of attack. Simulation parameters are shown in Table 4.6.

The angle of attack of the airfoil increases rapidly by a magnitude of $\triangle \alpha=\frac{\alpha_{0}}{2}$. Wagner's function is approximated as:

$$
\Phi(\tau)=\frac{\tau+2}{\tau+4}, \quad \tau>0
$$

where $\tau$ is the non-dimensional time, given as:

$$
\tau=\frac{2 U}{c} t
$$

The increase in lift of the airfoil due to the sudden increase in pitch angle is derived by Wagner as [2]:

$$
\Delta L=\frac{1}{2} \rho V_{0}^{2} c c_{l_{\alpha}} \Delta \alpha \Phi(\tau)
$$

Table 4.6: CFD simulation parameters for Wagner's motion.

\begin{tabular}{|c|c|c|}
\hline Parameter & Trial 1 & Trial 2 \\
\hline Initial pitch $\theta_{0}$ & $0 \mathrm{deg}$ & $0 \mathrm{deg}$ \\
\hline Final pitch $\theta$ & $8 \mathrm{deg}$ & $5 \mathrm{deg}$ \\
\hline Wind speed $V_{0}$ & $25 \frac{\mathrm{m}}{\mathrm{s}}$ & $50 \frac{\mathrm{m}}{\mathrm{s}}$ \\
\hline Simulation runtime & $3 \mathrm{~s}$ & $1.5 \mathrm{~s}$ \\
\hline Pitch transition time $t_{1}$ & $1.5 \mathrm{~s}$ & $0.75 \mathrm{~s}$ \\
\hline
\end{tabular}


ANSYS Fluent cannot handle a sudden change in pitch angle without experiencing a singularity as well as negative cell volume in the mesh due to excessive mesh deformation. As such, Wagner's solution for the airfoil was defined using, [2].

$$
\theta(t)=\theta_{0}+\triangle \theta \frac{1}{\pi}\left[\tan ^{-1}\left(75\left(t-t_{1}\right)\right)+\frac{\pi}{2}\right]
$$

Lift coefficient results from ANSYS Fluent and the 2D Panel Method are shown in Figures 4.21 and 4.22. Comparison of results from ANSYS Fluent and the 2D Panel Method show good agreement. Discrepancies can be observed. The 2D Panel Method lift results are slightly higher than Fluent results. This can be attributed to the mesh quality as well as the difference between the ANSYS pressure-based solver and the 2D Panel Method.

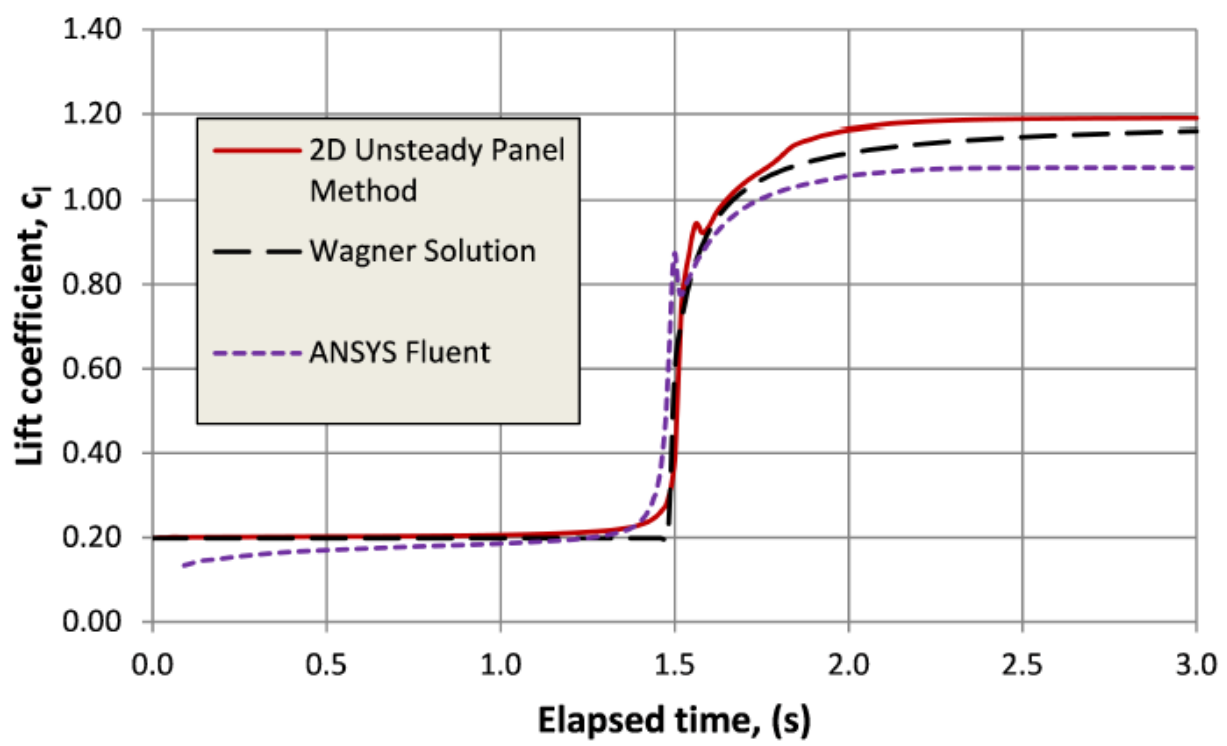

Figure 4.21: Comparison of Wagner's function lift coefficient results, $\triangle \theta=8 \mathrm{deg}$ and $V_{0}=50 \frac{\mathrm{m}}{\mathrm{s}}$ [2]. 


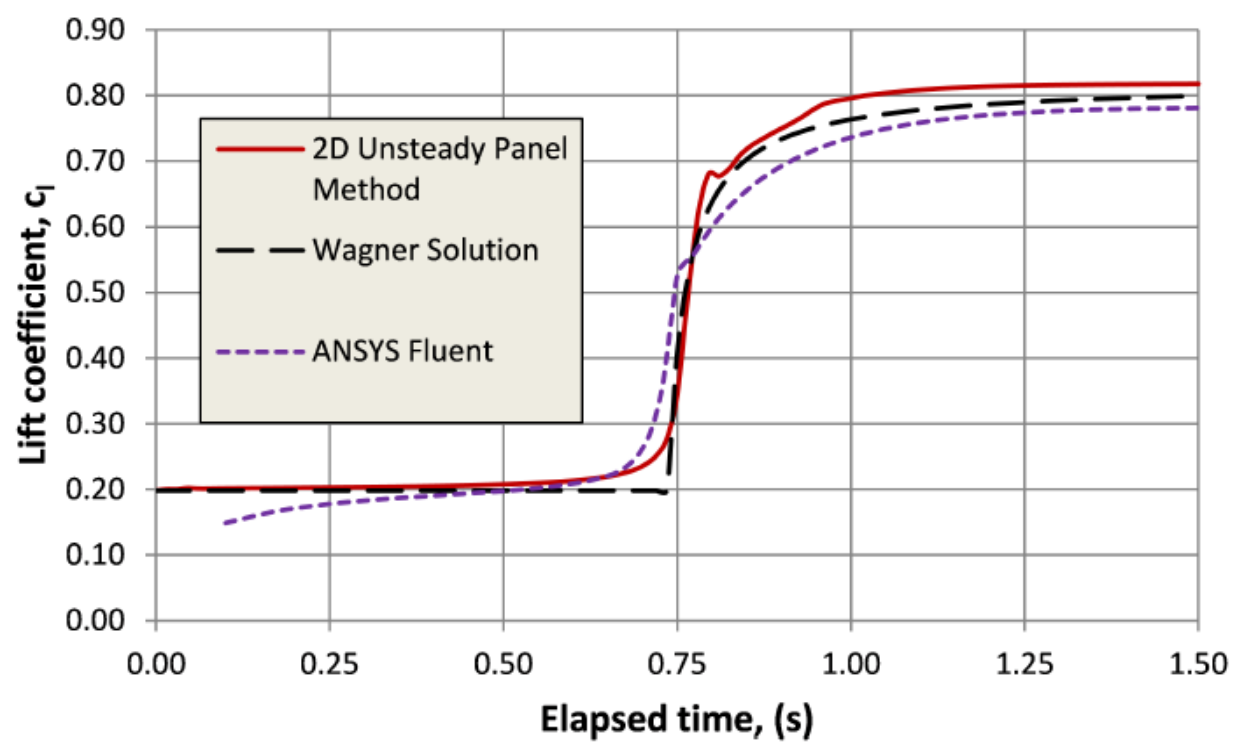

Figure 4.22: Comparison of Wagner's function lift coefficient results, $\triangle \theta=5 \mathrm{deg}$ and $V_{0}=25 \frac{\mathrm{m}}{\mathrm{s}}[2]$.

\subsection{D CFD and FEA Analysis}

Having validated the accuracy of the 2D Unsteady Panel method in the PAAC-WTB algorithm, the next step was to validate the Beam Kinematics solver portion of the code. The beam kinematics solver effectively simplifies the 3D nature of the geometry into a 2D linear cross sectional analysis and a 1D non-linear model for deformations, based on the Variational Asymptotic Method [1]. For the 1D analysis portion, the Ritz method $[2,29]$ is used to derive linear ordinary differential equations of motion for the structural deformation of the blade. By assuming a combination of mode shapes for the blade deflection, Euler-Lagrange equations are then used to determine the equations of motion [2]. Several test cases were set up to determine the accuracy of the solver's modal response analysis and the forced response structural analysis. Results were compared against results from ANSYS Workbench and classical vibration solutions. For the purpose of this thesis, all FEA structural dynamic analyses performed are linear analyses, with linear material properties. 
Table 4.7: Aluminum tube material properties and dimensions.

\begin{tabular}{|c|c|}
\hline Parameter & Value \\
\hline Length & $0.5 \mathrm{~m}$ \\
\hline Diameter & $0.05 \mathrm{~m}$ \\
\hline Thickness & $0.002 \mathrm{~m}$ \\
\hline Density $\rho$ & $2500 \frac{\mathrm{kg}}{\mathrm{m}^{3}}$ \\
\hline Young's Modulus E & $70 \times 10^{9} \frac{\mathrm{N}}{\mathrm{m}^{2}}$ \\
\hline Shear Modulus G & $26 \times 10^{9} \frac{\mathrm{N}}{\mathrm{m}^{2}}$ \\
\hline
\end{tabular}

\subsubsection{Modal Response and Forced Response Analysis}

Two cases were examined for both the modal response and the forced response structural solver: an aluminum tube and the S809 blade used in the wind tunnel experiment with the tapered root removed.

\subsubsection{Analysis of Aluminum Tube}

A uniform isotropic circular aluminum tube, as shown in Figure 4.23, was modelled in ANSYS Workbench with the parameters defined in Table 4.7. The tube was analysed for the first six modes and for tip deflection due to a transient load function applied at the tip with one fixed end.

The natural frequencies for the first six modes were determined using both ANSYS Workbench and the Beam Kinematics solver in PAAC-WTB and compared as shown in Table 4.8. The frequencies are comparatively similar, with the best agreement for the first and second bending modes. One observation is that the RayleighRitz modal solver that the algorithm uses has less freedom compared to the ANSYS FEA model, which makes it more stiff. This results in higher natural frequency values 
Table 4.8: Aluminum tube modal response natural frequencies.

\begin{tabular}{|c|c|c|}
\hline Mode & ANSYS frequencies (Hz) & Solver frequencies $\mathbf{H z})$ \\
\hline 1st bending & 202 & 210 \\
\hline 2nd bending & 1258 & 1326 \\
\hline 1st torsional & 1596 & 1780 \\
\hline 1st extensional & 2757 & 2917 \\
\hline
\end{tabular}

obtained from the solver.

For the forced response structural analysis, the sinusoidal tip load function applied is given by,

$$
F(t)=1000 \cos (2 \pi f t)
$$

where $f$ is the designated forcing frequency of the load and $t$ is the solution runtime.

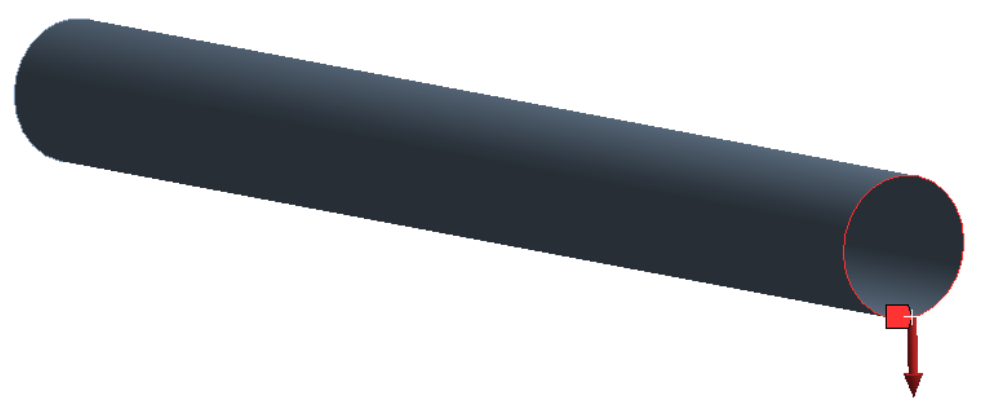

Figure 4.23: Aluminum tube with applied tip load.

The response of the tube, specifically the tip deflection, was determined again using ANSYS Workbench and the Kinematics Beam solver. The analysis was performed at three different forcing frequencies and with no damping defined for the tube. Figures 4.24, 4.25, and 4.26 show the tip deflections at each frequency. Good 
agreement is observed between results from ANSYS and the solver. Discrepancies can be attributed to the difference between ANSYS's structural solver and the algorithm's Beam Kinematics Solver.

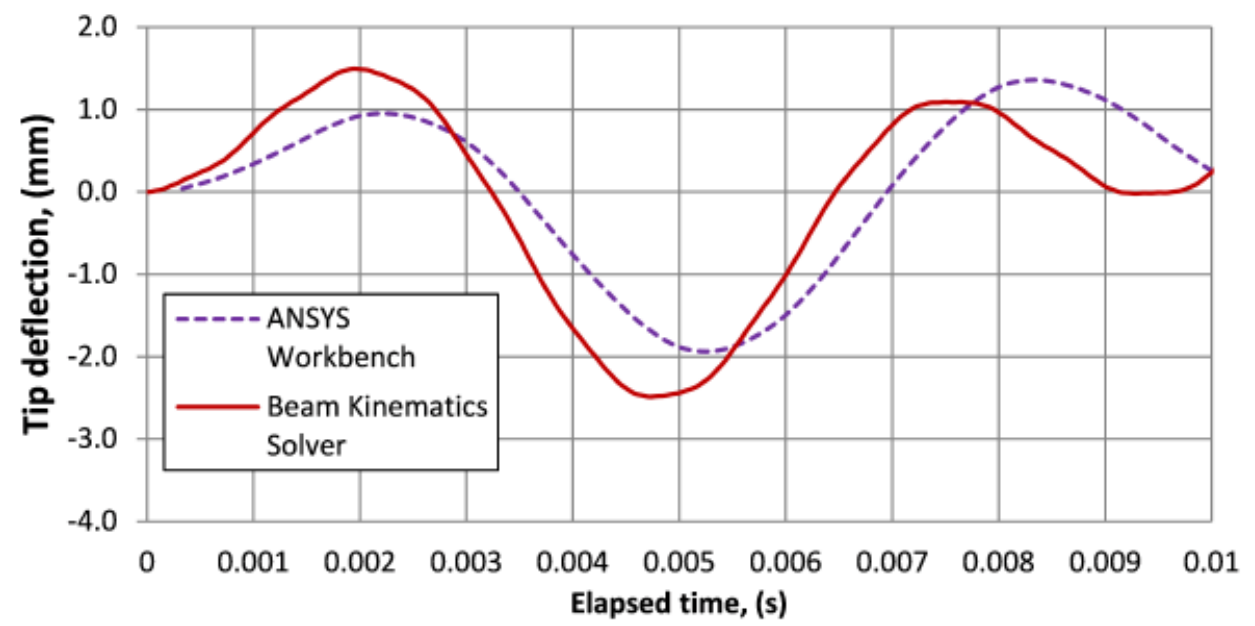

Figure 4.24: Aluminum tube tip deflection at $f=100 \mathrm{~Hz}$ [2].

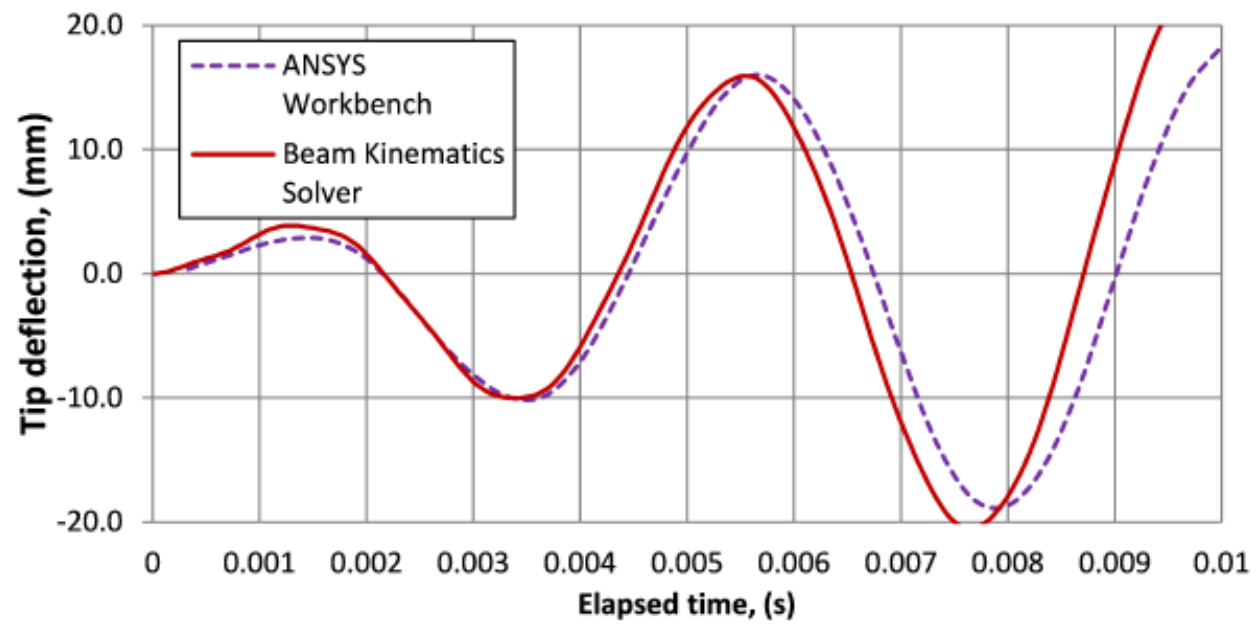

Figure 4.25: Aluminum tube tip deflection at $f=250 \mathrm{~Hz}[2]$. 


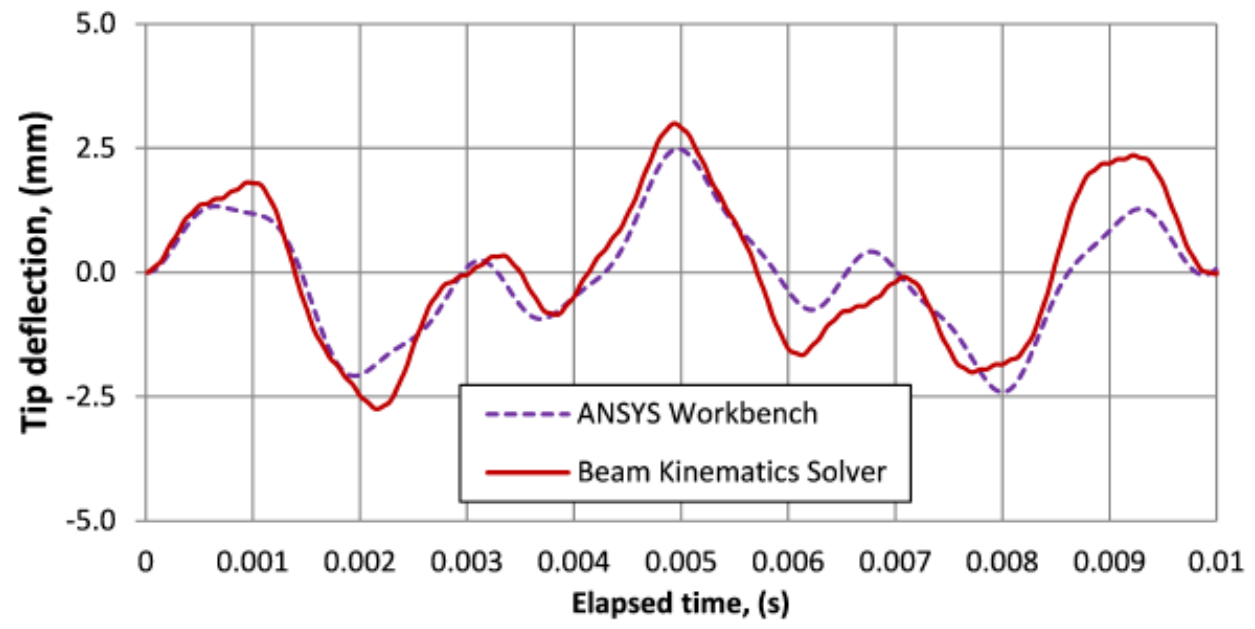

Figure 4.26: Aluminum tube tip deflection at $f=500 \mathrm{~Hz}[2]$.

\subsubsection{Analysis of the S809 Blade}

The same set of analyses was performed on the S809 blade to investigate the Beam Kinematics solver's accuracy when dealing with more complicated geometry. The S809 blade model used along with the dimensions and the assigned material properties are shown in Table 4.9. The modal response for the first four modes of the S809 blade was determined from ANSYS and the solver and are compared in Table 4.10.

Major discrepancies can be observed between the corresponding values. This is attributed to the fixed end constraint approachused by ANSYS in comparison to the Beam Kinematic solver. In ANSYS, the constraint is defined around the complete boundary of one end of the blade, while the solver defines the constraint at a single point [2].

For the forced response analysis, a distributed sinusoidal pressure load, given by Equation 4.16, was applied on the top surface of the S809 blade as shown in Figure 4.27 
Table 4.9: S809 blade model material properties and dimensions.

\begin{tabular}{|c|c|}
\hline Parameter & Value \\
\hline Length & $0.539 \mathrm{~m}$ \\
\hline Chord & $0.127 \mathrm{~m}$ \\
\hline Thickness & $0.0015 \mathrm{~m}$ \\
\hline Density $\rho$ & $930 \frac{\mathrm{kg}}{\mathrm{m}^{3}}$ \\
\hline Young's Modulus E & $1700 \mathrm{MPa}$ \\
\hline Tensile strength & $48 \mathrm{MPa}$ \\
\hline Poisson's ratio & 0.4 \\
\hline
\end{tabular}

Table 4.10: S809 modal response natural frequencies.

\begin{tabular}{|c|c|c|}
\hline Mode & ANSYS frequencies $\mathbf{( H z )}$ & Solver frequencies $\mathbf{( H z})$ \\
\hline 1 & 16.4 & 23.1 \\
\hline 2 & 30.8 & 50.6 \\
\hline 3 & 91.2 & 110.6 \\
\hline 4 & 138 & 228 \\
\hline
\end{tabular}

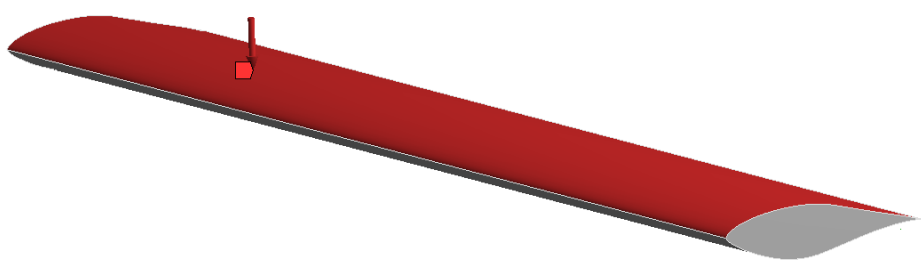

Figure 4.27: S809 blade with applied distributed pressure load. 


$$
P(z, t)=25 \cos (2 \pi f t)
$$

The analysis was repeated twice at two different forcing frequencies of $f_{n}=0.6 f_{n, 1}$ $\mathrm{Hz}$ and $f=1.2 f_{n, 2} \mathrm{~Hz}$, with no damping predefined. Figures 4.28 and 4.29 show the tip deflections at each frequency.

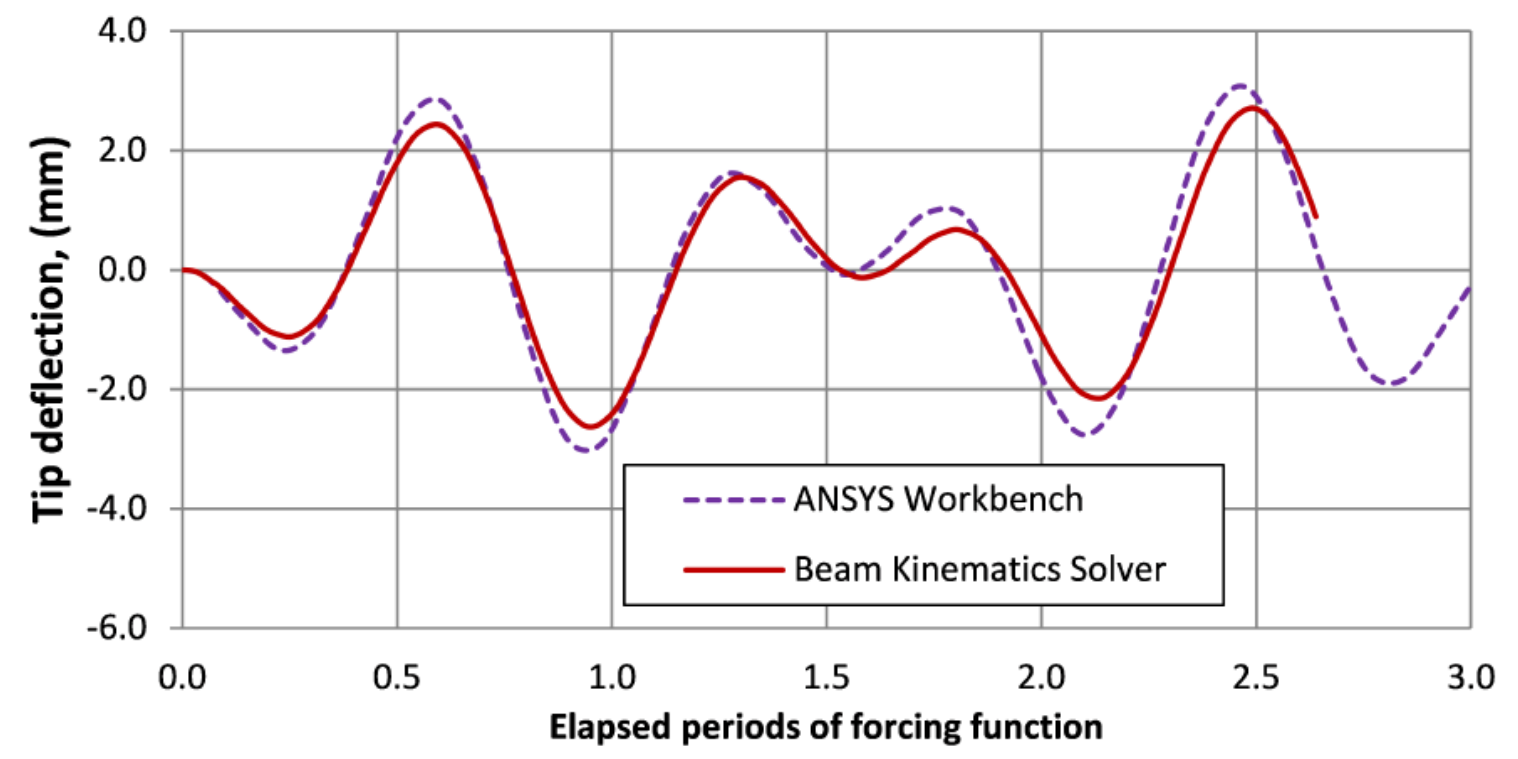

Figure 4.28: S809 tip deflection at $f=0.6 f_{n, 1}[2]$.

Good agreement is observed between tip deflection data from both programs. Tip deflection results from the Beam Kinematics solver are lower in comparison which is due to its higher predicted first fundamental frequency, since the solver's model is stiffer [2]. 


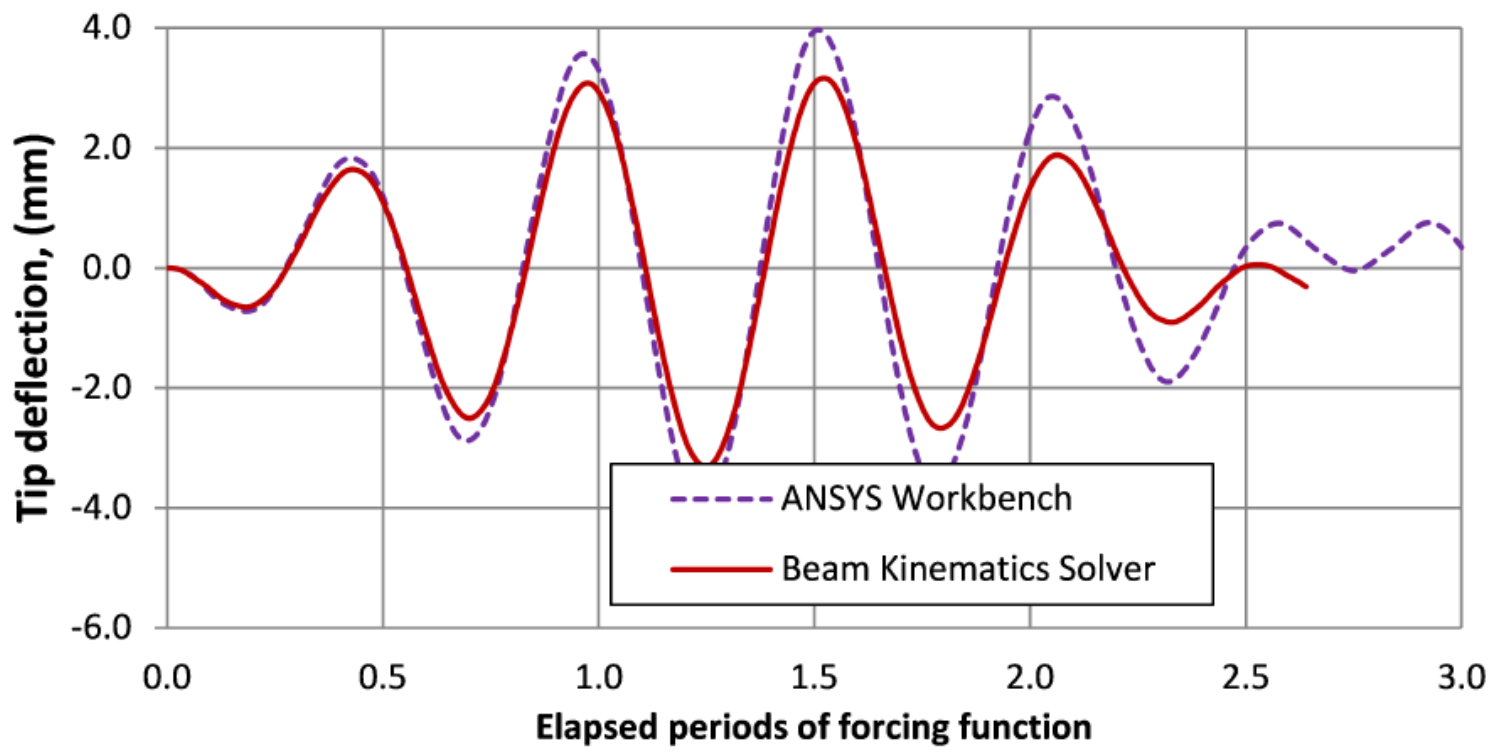

Figure 4.29: S809 tip deflection at $f=1.2 f_{n, 1}[2]$

\subsubsection{D Aeroelastic Analysis}

Once the accuracy and validity of the individual algorithm components of the PAACWTB package were determined, an aeroelastic simulation on the S809 blade was carried out to investigate the validity of the PAAC-WTB algorithm as a complete package. The aeroelastic analysis is implemented using the two way fluid/structure interaction (FSI) scheme in ANSYS Workbench, by coupling the Fluent module (FM) and the Transient Structural module (TSM), as shown in Figure 4.30. Both the FM and TSM are attached to the System Coupling module (SCM), which allows the user to access the two way FSI scheme.

The SCM requires the user to define the parts of the model on which the FSI analysis will be performed. For this analysis, all surfaces on the S809 blade were selected as the fluid/solid interface in both the TSM and the FM, as shown in Figures 4.31 .

The two modules share data throughout the simulation runtime at each defined 


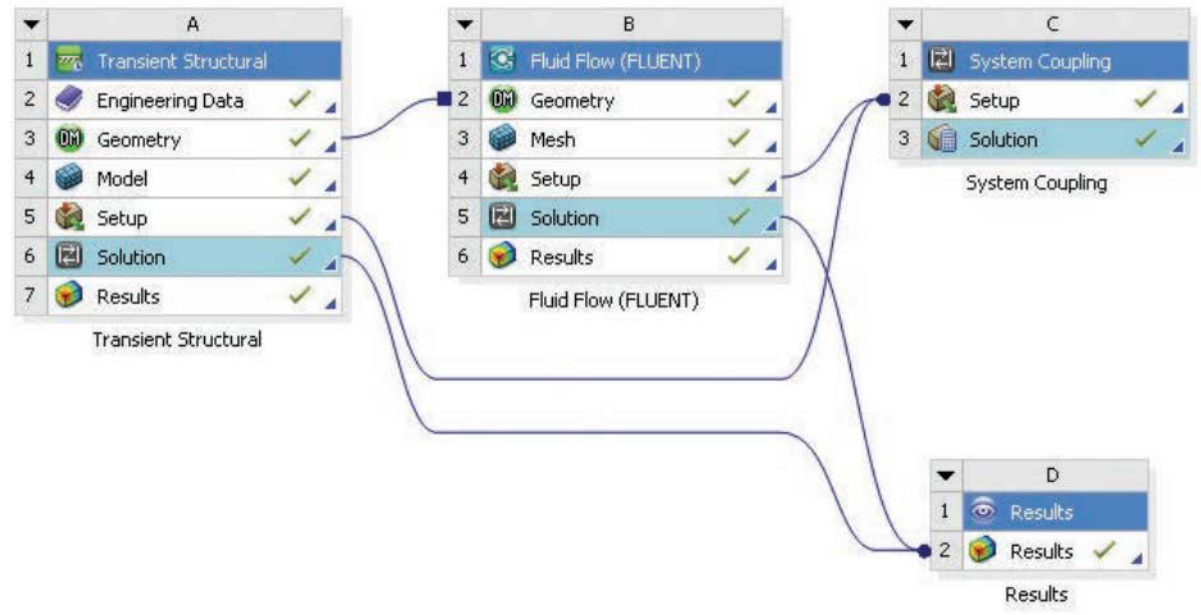

Figure 4.30: Two way fluid/structure ANSYS Workbench setup.

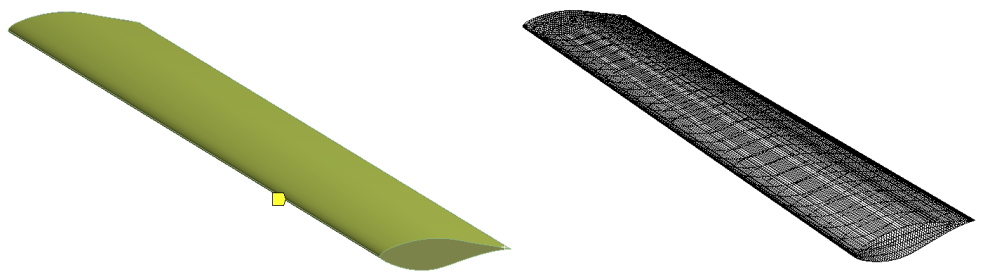

Figure 4.31: S809 blade fluid solid interface in TSM (left) and FM (right). 
timestep, until the end time is reached. Force data from the pressure applied on the blade due to the airflow is transferred from the FM to the TSM. The TSM then computes the displacement of the blade and deforms the surrounding mesh accordingly.

\begin{tabular}{|c|c|c|c|}
\hline \multicolumn{3}{|c|}{ Properties of DataTransfer : Data Transfer } & \multirow{2}{*}{$4 \times$} \\
\hline & A & B & \\
\hline 1 & Property & Value & \\
\hline 2 & - Source & & \\
\hline 3 & Participant & FLUENT & 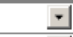 \\
\hline 4 & Region & fluidbladeint & 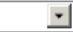 \\
\hline 5 & Variable & force & -1 \\
\hline 6 & - Target & & \\
\hline 7 & Participant & Transient Structural & 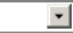 \\
\hline 8 & Region & Fluid Solid Interface & 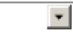 \\
\hline 9 & Variable & Force & 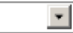 \\
\hline 10 & - Data Transfer Control & & \\
\hline 11 & Transfer At & Start Of Iteration & 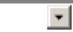 \\
\hline 12 & Under Relaxation Factor & 1 & \\
\hline 13 & Convergence Target & 0.01 & \\
\hline
\end{tabular}

\begin{tabular}{|c|c|c|c|}
\hline Prope & is of DataTransfer : Data Transfe & & $=4 \times$ \\
\hline & A & B & \\
\hline 1 & Property & Value & \\
\hline 2 & I Source & & \\
\hline 3 & Participant & Transient Structural & - \\
\hline 4 & Region & Fluid Solid Interface & - \\
\hline 5 & Variable & Incremental Displacement & - \\
\hline 6 & - Target & & \\
\hline 7 & Participant & FLUENT & - \\
\hline 8 & Region & fluidbladeint & 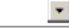 \\
\hline 9 & Variable & displacement & - \\
\hline 10 & - Data Transfer Control & & \\
\hline 11 & Transfer At & Start Of Iteration & - \\
\hline 12 & Under Relaxation Factor & 1 & \\
\hline 13 & Convergence Target & 0.01 & \\
\hline
\end{tabular}

Figure 4.32: Force and displacement data transfer between the TSM and the FM.

The TSM then transfers the displacement of the blade to the FM, which recalculates the pressure on the displaced blade at the next time step. The process then repeats itself until the user-assigned simulation runtime is completed or excessive mesh deformation occurs. The two way transfer of data was done by assigning data transfer requirements from the TSM fluid solid interface to the FM fluid solid interface and vice versa, as shown in Figure 4.32, to transfer data throughout the simulation runtime.

For the meshing process, multiple mesh enclosures were applied around the blade, as shown in Figure 4.33, each with a different mesh grid. The smallest enclosure around the blade, shown in Figure 4.34, composed of free form quadrilateral and triangular elements with the highest element count, to obtain the most accurate pressure data from the blade. This region was also expected to experience the largest degree of deformation. 

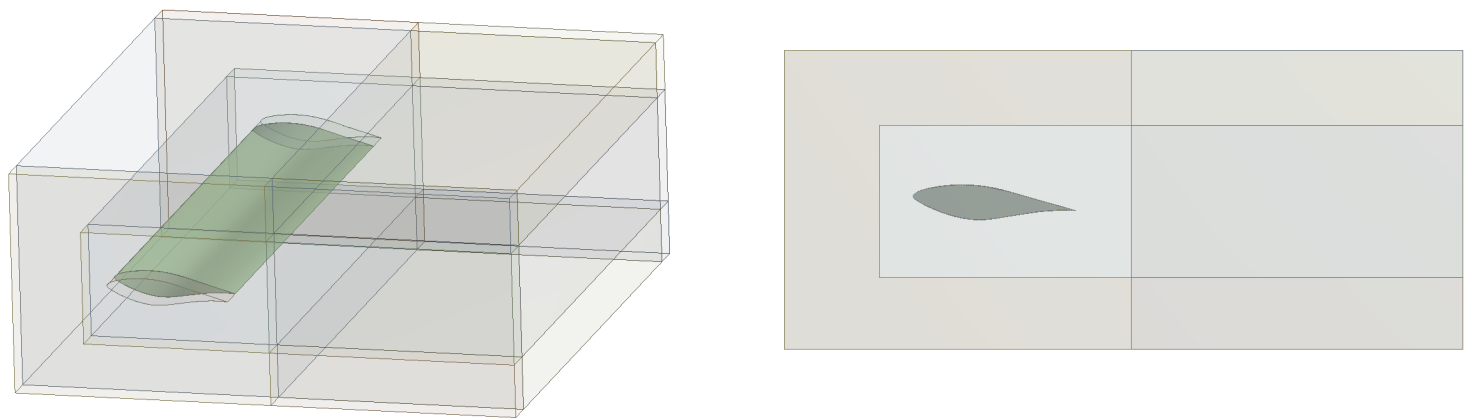

Figure 4.33: Mesh enclosures around S809 blade.

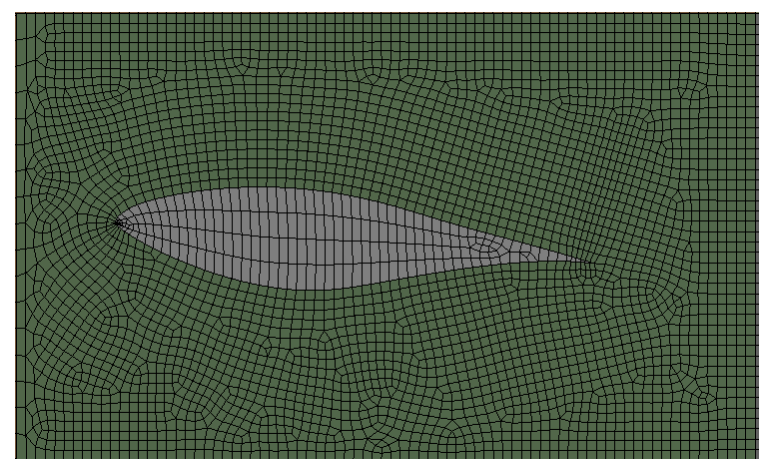

Figure 4.34: First mesh enclosure around the S809 blade.

Moving away from the blade upstream, above, and below the blade, the second enclosure mesh, shown in Figure 4.35, transitions from free form quadrilaterals and triangular elements to structured quadrilaterals albeit with lower element count to reduce simulation runtime.

Downstream of the blade, the mesh enclosure, shown in Figure 4.36, is a structured quadrilateral mesh with a high element count to ensure accuracy when analysing the wake. Figures 4.37 and 4.38 show the meshed enclosures around the airfoil at 0 and 5 deg angle of attack respectively. 


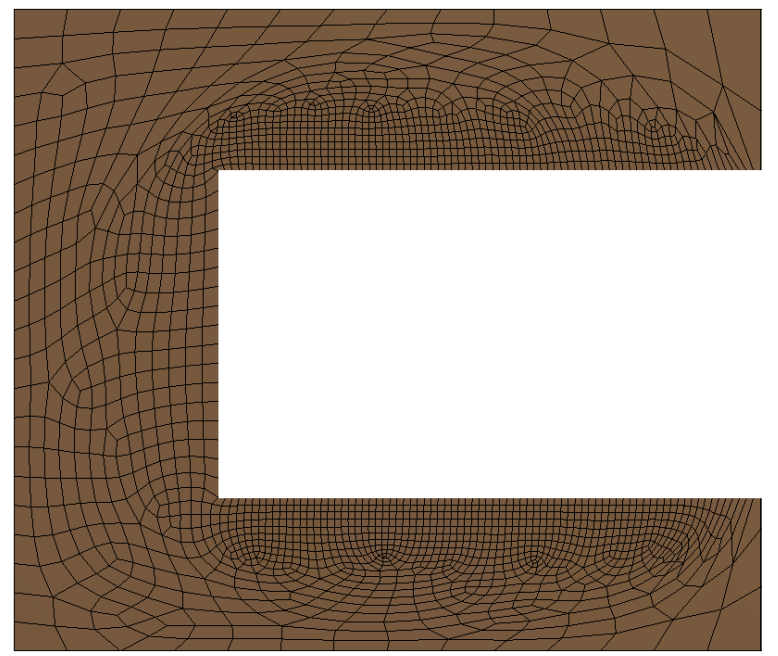

Figure 4.35: Second mesh enclosure, upstream and around the S809 blade.

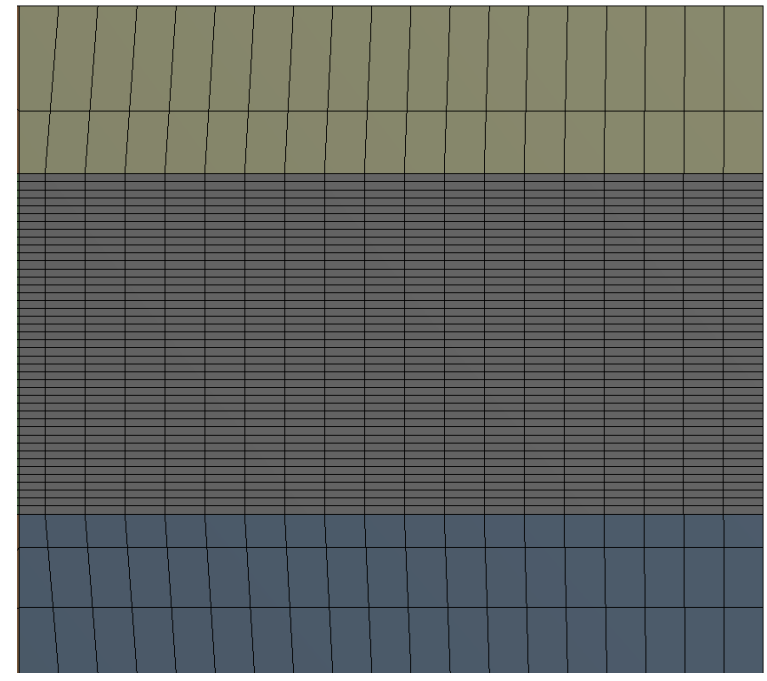

Figure 4.36: Third mesh enclosure, downstream of S809 blade. 


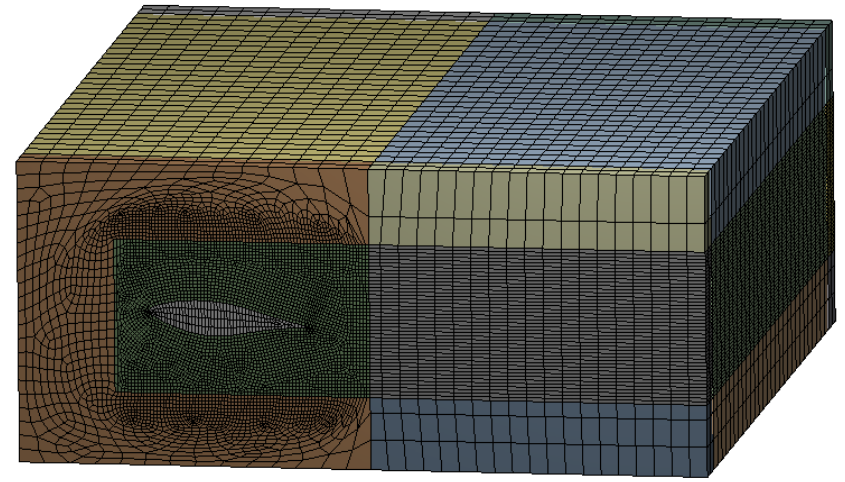

Figure 4.37: Aeroelastic mesh around S809 blade, 5 deg pitch angle.

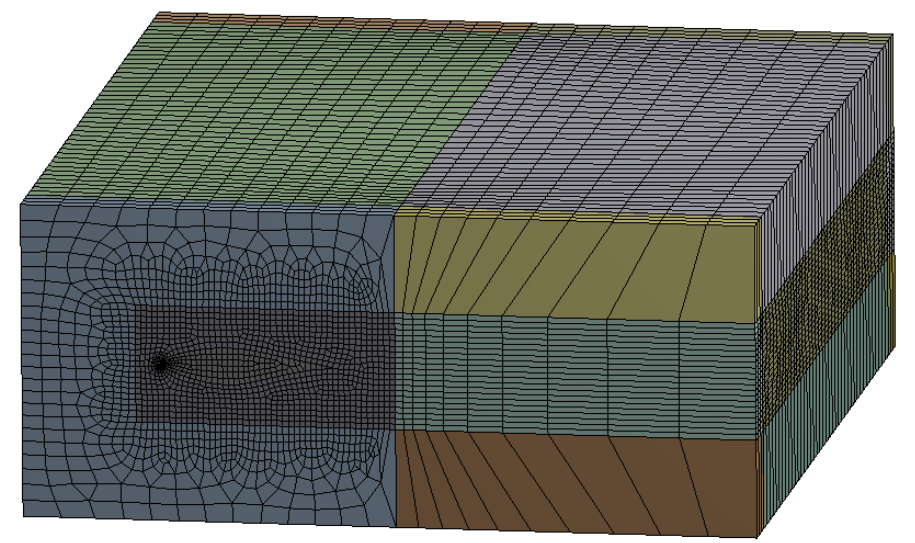

Figure 4.38: Aeroelastic mesh around S809 blade, 0 deg pitch angle. 
The boundary conditions for the mesh were then defined in the FM for the CFD portion of the analysis. The velocity inlet and pressure outlet were defined on the surfaces of the enclosure upstream and downstream of the blade respectively, as shown in Figure 4.39. The velocity at the inlet was defined for each analysis trial, while the pressure outlet was kept the same for all three trials. The parameters used are in Table 4.11. Rigid wall boundary conditions were applied on the remaining enclosure surfaces, shown in Figure 4.40.

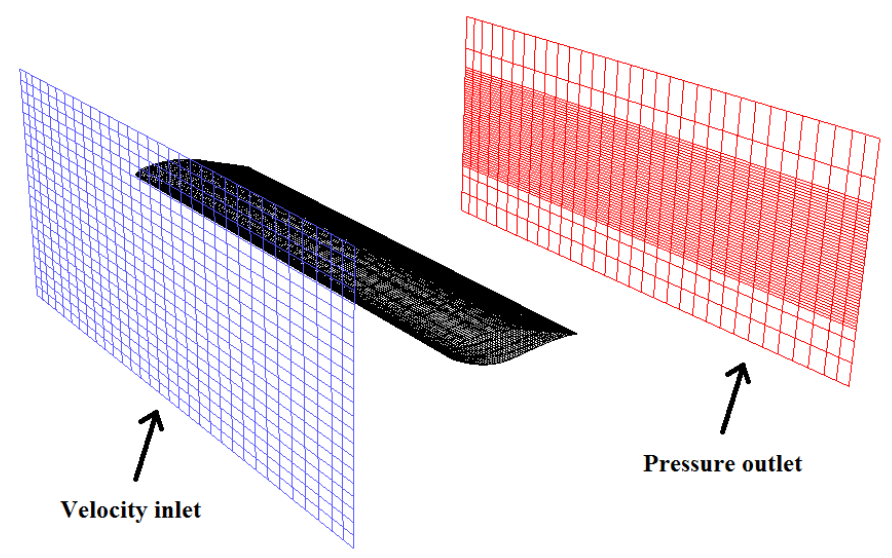

Figure 4.39: Aeroelastic mesh inlet and outlet boundary conditions.

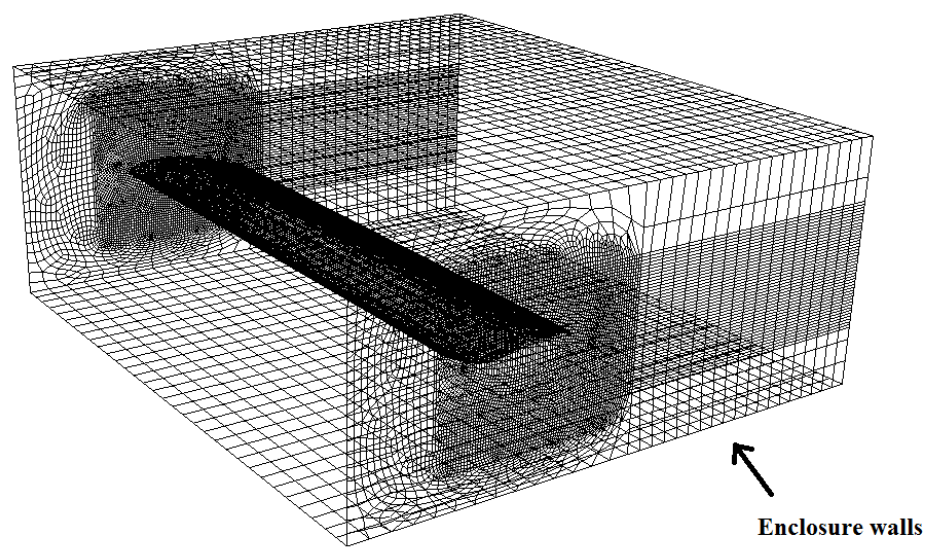

Figure 4.40: Aeroelastic mesh wall boundary conditions. 
For the structural portion of the analysis, a structural mesh of the S809 model was created and a fixed end boundary condition was applied to one end of the blade, as shown in Figure 4.41.
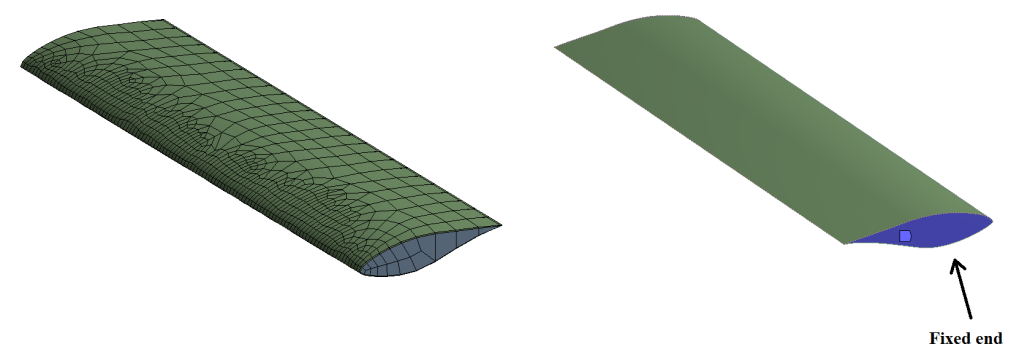

Figure 4.41: TSM mesh and fixed end boundary constraint.

The geometric dimensions and material properties of the S809 blade used for the analysis are shown in Table 4.9. Three trials were performed on the blade, with different parameters. Trial parameters are shown in Table 4.11.

Table 4.11: Simulation parameters for aeroelastic trials.

\begin{tabular}{|c|c|c|c|}
\hline Parameter & Trial 1 & Trial 2 & Trial 3 \\
\hline Wind speed $V_{0}$ & $15 \frac{\mathrm{m}}{\mathrm{s}}$ & $17.3 \frac{\mathrm{m}}{\mathrm{s}}$ & $19.7 \frac{\mathrm{m}}{\mathrm{s}}$ \\
\hline Angle of attack $\alpha$ & $0 \mathrm{deg}$ & $5 \mathrm{deg}$ & $5 \mathrm{deg}$ \\
\hline $\begin{array}{c}\text { Pressure at outlet } \\
\text { hline Time step size }\end{array}$ & $0.001 .325 \mathrm{kPa}$ & $101.325 \mathrm{kPa}$ & $101.325 \mathrm{kPa}$ \\
\hline Number of timesteps & 100 & 0.001 & 0.001 \\
\hline End time & $0.1 \mathrm{~s}$ & $0.15 \mathrm{~s}$ & $0.15 \mathrm{~s}$ \\
\hline Mesh element count & 279,000 & 237,000 & 237,000 \\
\hline
\end{tabular}

Tip deflection results, shown in Figure 4.42, results were obtained from the TSM for all three trials performed and were compared against results from the PAAC-WTB algorithm, as shown in Figures 4.43, 4.44, and 4.45. 


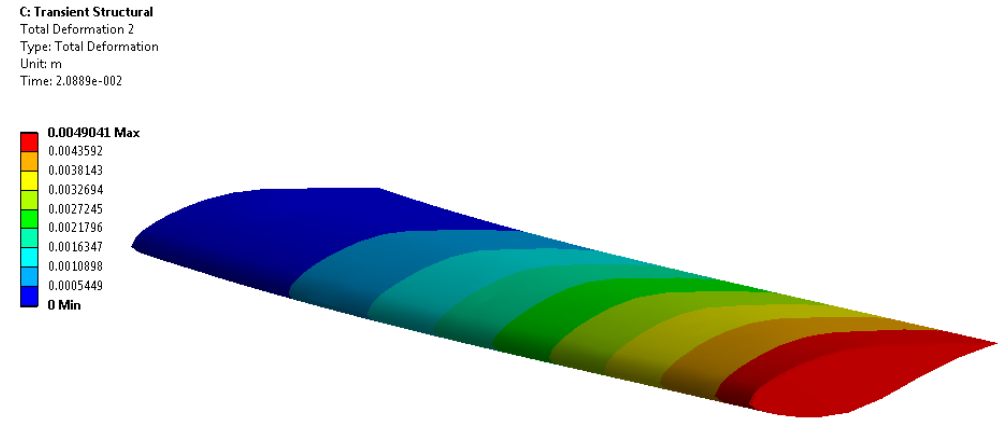

Figure 4.42: Aeroelastic analysis S809 blade tip deflection.

Due to excessive mesh deformation causing simulation crashes, the collected results were limited to half the intended simulation duration. Nonetheless, the results show reasonable agreement with minor discrepancy. Differences in results between ANSYS and PAAC-WTB can be attributed to the different fluids and structural solvers ANSYS uses in comparison to the PAAC-WTB algorithm.

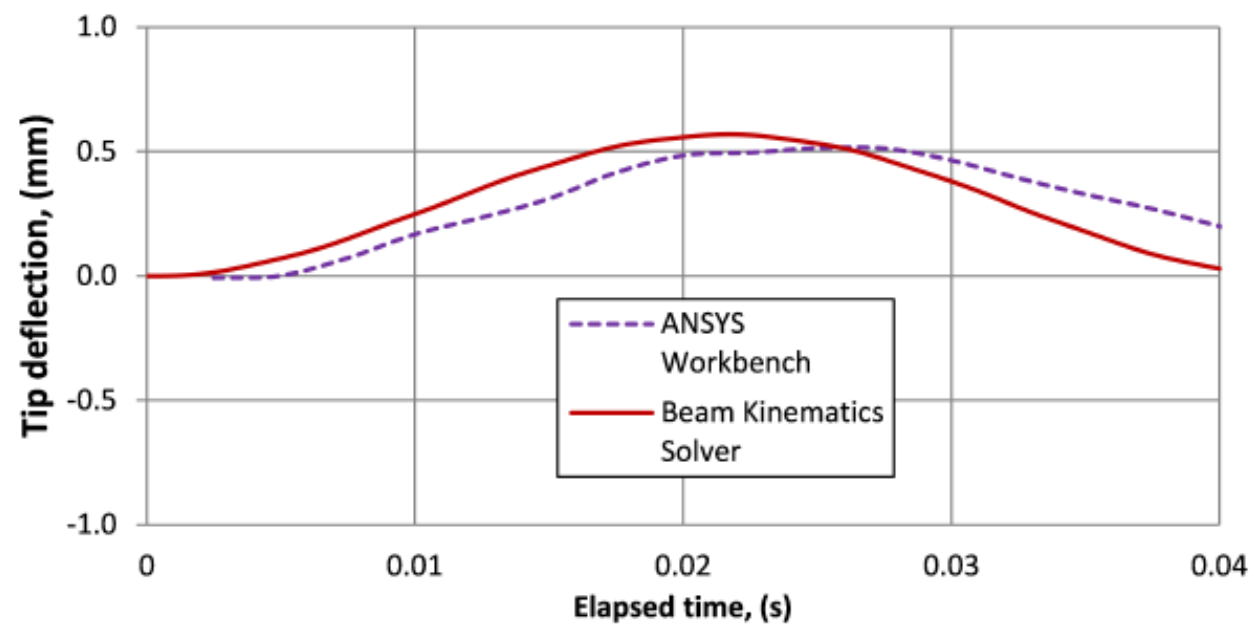

Figure 4.43: S809 aeroelastic results, $V_{0}=15 \frac{\mathrm{m}}{\mathrm{s}}, \alpha=0 \mathrm{deg}$. 


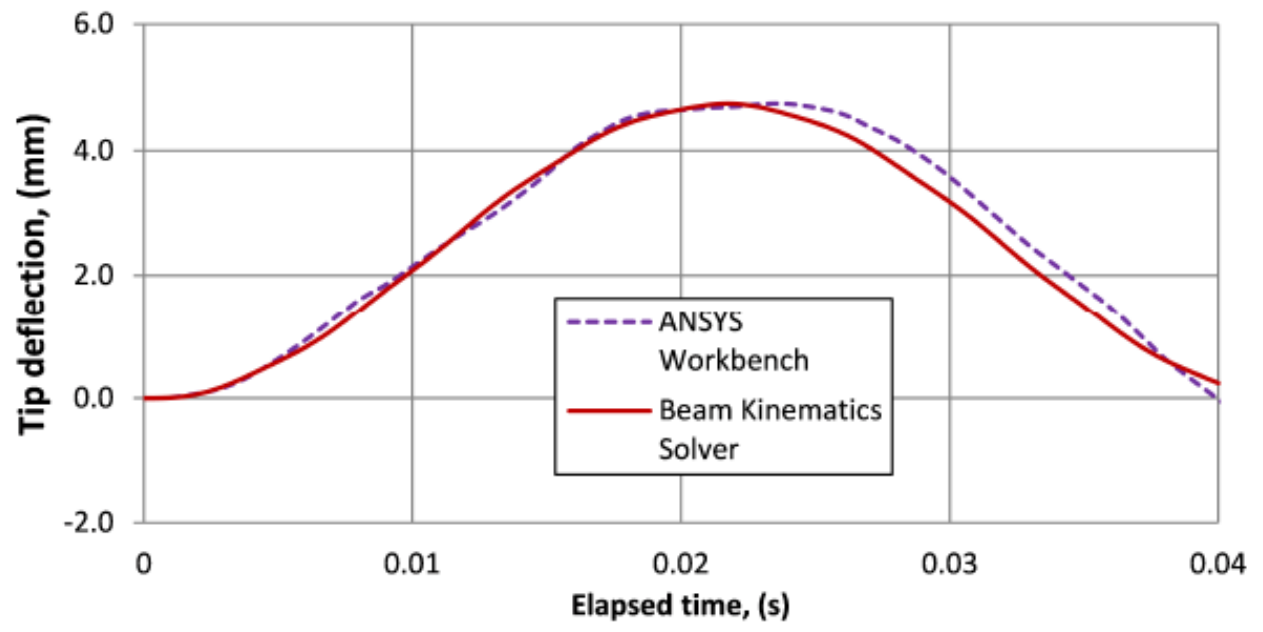

Figure 4.44: S809 aeroelastic results, $V_{0}=17.3 \frac{\mathrm{m}}{\mathrm{s}}, \alpha=5 \mathrm{deg}$.

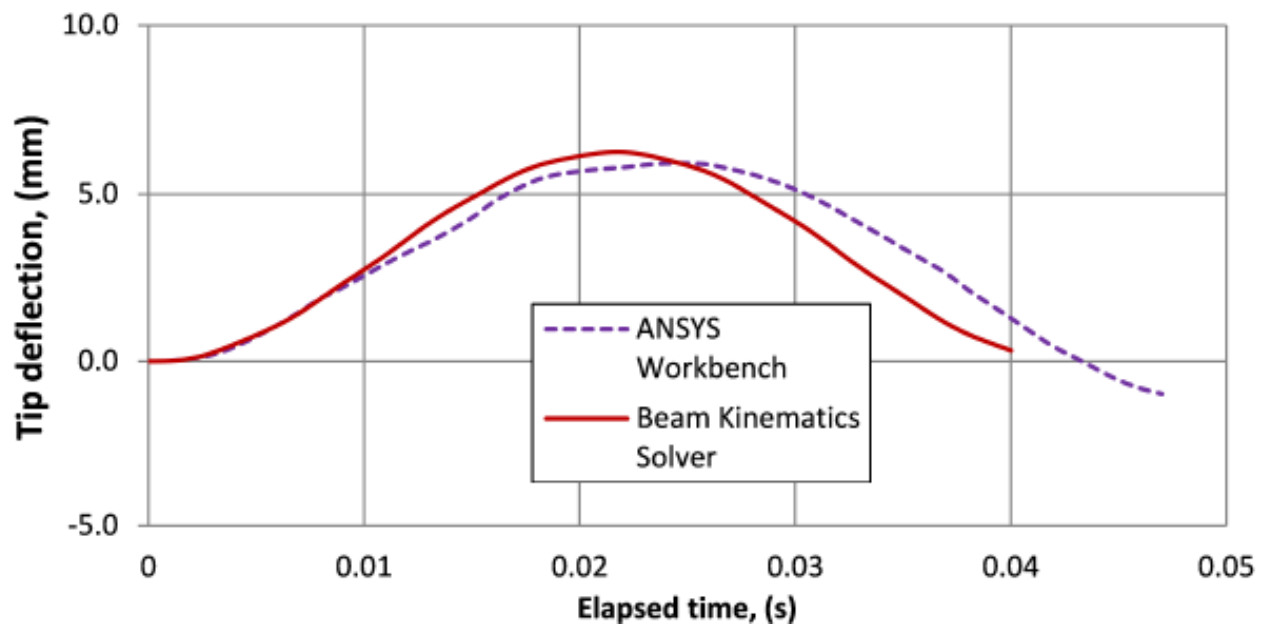

Figure 4.45: S809 aeroelastic results, $V_{0}=19.7 \frac{\mathrm{m}}{\mathrm{s}}, \alpha=5 \mathrm{deg}$. 


\subsection{Mesh Convergence}

In order to determine whether the quality of the mesh affects aerodynamic analysis of the blade model performed using Fluent, a mesh convergence comparison was performed. The mesh enclosure around the blade was selected for the comparison. Four different element sizes were selected, shown in Table 4.12.

Table 4.12: Simulation parameters for mesh convergence comparison.

\begin{tabular}{|c|c|c|c|c|}
\hline Parameter & Mesh 1 & Mesh 2 & Mesh 3 & Mesh 4 \\
\hline Wind speed $V_{0}$ & $17.3 \frac{\mathrm{m}}{\mathrm{s}}$ & $17.3 \frac{\mathrm{m}}{\mathrm{s}}$ & $17.3 \frac{\mathrm{m}}{\mathrm{s}}$ & $17.3 \frac{\mathrm{m}}{\mathrm{s}}$ \\
\hline Angle of attack $\alpha$ & $5 \mathrm{deg}$ & $5 \mathrm{deg}$ & $5 \mathrm{deg}$ & $5 \mathrm{deg}$ \\
\hline Pressure at outlet & $101.325 \mathrm{kPa}$ & $101.325 \mathrm{kPa}$ & $101.325 \mathrm{kPa}$ & $101.325 \mathrm{kPa}$ \\
\hline Time step size & 0.001 & 0.001 & 0.001 & 0.001 \\
\hline Number of timesteps & 150 & 150 & 150 & 150 \\
\hline End time & $0.15 \mathrm{~s}$ & $0.15 \mathrm{~s}$ & $0.15 \mathrm{~s}$ & $0.15 \mathrm{~s}$ \\
\hline Mesh element size & $0.001 \mathrm{~m}$ & $0.002 \mathrm{~m}$ & $0.003 \mathrm{~m}$ & $0.004 \mathrm{~m}$ \\
\hline
\end{tabular}

Figure 4.46 shows all four meshes used with the differing element sizes. The lift coefficient was used as the convergence parameter to determine the significance of the mesh sizing on the aerodynamic analysis of the blade.

Table 4.13 shows all lift coefficient values obtained from all four meshes. The results indicate a $4.1 \%$ difference between meshes 1 and 2 in , $1.5 \%$ difference between meshes 2 and 3, and a 1.1\% difference between meshes 3 and 4 . Further decrease in element size produced negligible difference in lift coefficient value. This trend clearly demonstrates mesh convergence, with a 1.1\% difference between meshes 3 and 4, which indicates that the mesh was suitably refined. This also demonstrates the importance of mesh refinement to improve the accuracy of the aerodynamic analysis. 


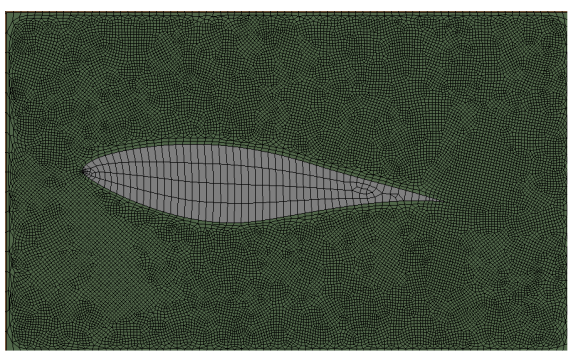

Mesh 1

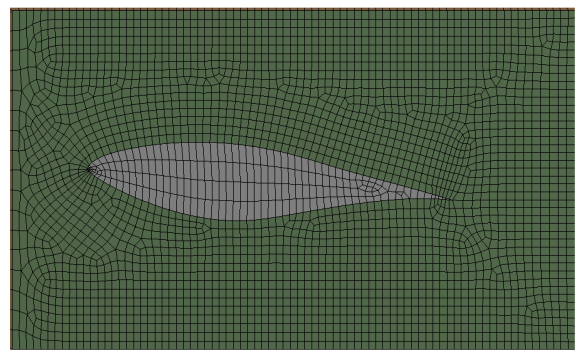

Mesh 3

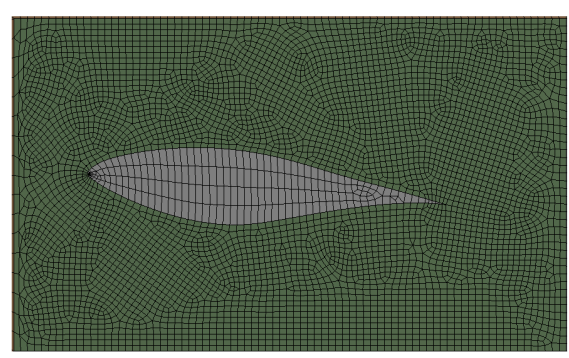

Mesh 2

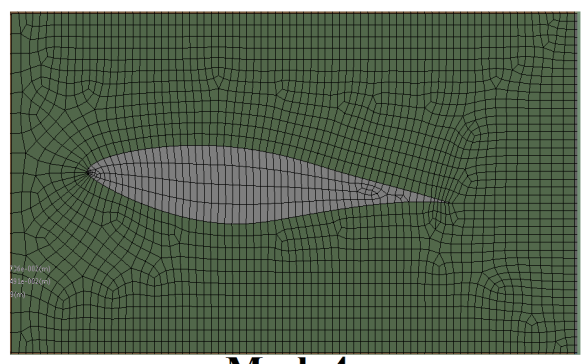

Mesh 4

Figure 4.46: Mesh 1 to 4 with varying element sizes.

Table 4.13: Lift coefficient mesh convergence results.

\begin{tabular}{|c|c|c|c|c|}
\hline Parameter & Mesh 1 & Mesh 2 & Mesh 3 & Mesh 4 \\
\hline Lift coefficient $C_{L}$ & 0.4826 & 0.5179 & 0.5258 & 0.5317 \\
\hline
\end{tabular}




\section{Chapter 5}

\section{Conclusion}

The validation methods proved to be successful. The single blade wind tunnel test results show some agreement when compared to the PAAC-WTB algorithm results produced by McFarlane [2]; however further correlation could not be established due to the existence of several factors that apply to both the algorithm and the experiment, which include:

- The potential excitation of the blade support causing discrepancies in results

- The lack of turbulence modelling in the PAAC-WTB algorithm

- The quality of the manufactured S809 blade. The defects and gaps on the blade may have affected the excitation frequencies of the blade in comparison to the PAAC-WTB S809 blade model.

The ANSYS CFD and FEA analysis, both 2D and 3D, also proved to be successful. Results obtained from the PAAC-WTB algorithm show good agreement with data obtained from the CFD and FEA analyses.

This confirms the PAAC-WTB as a legitimate preliminary aeroelastic code that is suitable for further implementation. The CFD and FEA validation results were more conclusive in determining the accuracy of the algorithm compared to the wind 
tunnel experiment results; however, the experiment was considered to be a preliminary benchmark attempt and thus serves its purpose of providing a solid basis for further development of wind tunnel testing.

\subsection{Future Work and Recommendations}

The methods employed in this thesis are considered an initial stage in developing and require a comprehensive horizontal axis wind turbine validation process. The following aspects should be further investigated:

1. Improvement of the single non-rotating blade wind tunnel experiment to identify possible factors that influence results.

2. Development of the HAWT wind tunnel experiment to obtain validation data for the aeroelastic response of a three blade wind turbine rotor.

3. Implementation of further CFD and FEA analyses that include turbulence modelling for both 2D and 3D analysis. Also, implementation of a complete CFD and FEA HAWT rotor analysis in ANSYS, that includes more intricate features, such as rotating meshes and flow fields.

\subsection{Scientific Contribution}

This thesis presents a solid validation process for validating preliminary wind turbine areoelastic algorithms using ANSYS CFD and FEA analysis as well as the two way FSI analysis. An initial attempt in using an experimental validation method is also presented. Validation of such efficient and fast algorithms are essential to ensure the validity of their results during the initial stages of the design of wind turbines. 


\section{References}

[1] F. Khouli, Modelling and Attenuation Feasibility of the Aeroelastic Response of Active Helicopter Rotor Systems During the Engagement/Disengagement Phase of Maritime Operation. PhD thesis, Carleton University, May 2009.

[2] A. Mcfarlane, "An Algorithm for Preliminary Aeroelastic Analysis of Composite Wind Turbine Blades," Master's thesis, Carleton University, 2014.

[3] S. Gundtoft, "Wind Turbines." University College of Aarhus, 2009.

[4] O. Hansen, Aerodynamics of Wind Turbines. Earthscan USA, 2008.

[5] J. Mccosker, Design and Optimization of a Small Wind Turbine. publisher, 2012.

[6] W. D. H. Glauert, Aerodynamic Theory, ch. Airplane Propellors. SpringerVerlag, 1935.

[7] W. S. Wilson R., Lissaman P., Aerodynamic Performance of Wind Turbines. publisher, 1976.

[8] H. M. F. L. Simms D.A, Schreck S.J, "NREL unsteady aerodynamic experiment in the NASA-Ames wind tunnel: a comparison of predicitions to measurements," tech. rep., National Renewable Energy Laboratory, 2001.

[9] S. S. Sorensen N.N, Michelsen J.A, "Navier-STokes Predicitoons of the NREL Phase VI Rotor in the NASA Ames 80 by 120 Wind Tunnel," in ASME Conference Proceedings (editor, ed.), vol. 2002, pp. 94-105, year.

[10] D. M. Somers, "Design and Experimental Results for the S809 Airfoil," tech. rep., NREL, January 1997.

[11] J. Tangler, "NREL Airfoil Families for HAWTs," tech. rep., National Renewable energy Laboratory, 1995. 
[12] D. M. Somers, "Design and Experimental Results for the S809 Airfoil," tech. rep., Airfoils, Incorporated State College, National Renewable Energy Laboratory, 1997.

[13] B. Jonkman, "S809 airfoil." http://wind.nrel.gov/airfoils/shapes/S809. gif, October 2014.

[14] B. Jonkman, "S833 airfoil." http://wind.nrel.gov/airfoils/shapes/S833. gif, October 2014.

[15] B. Jonkman, "S834 airfoil." http://wind.nrel.gov/airfoils/shapes/S834. gif.

[16] B. Jonkman, "S835 airfoil." http://wind.nrel.gov/airfoils/shapes/S835. gif, October 2014.

[17] "Loctite 5 min epoxy technical data sheet." Henkel, 2014.

[18] M. P. Somers D., "The S833, S834 and S835 Airfoils," tech. rep., NREL, Nov 2001- Nov 2002.

[19] J. W. David Marten, QBlade Guidelines. Berlin Technical university, January 2013.

[20] S. Mctavish, Identification of Wind Turbine Testing Practices and Investigation of The Performance Benefits of Closely spaced Lateral Wind Farm Configurations. PhD thesis.

[21] J. A. P. Jewel B. Barlow, William H.Rae, Low Speed Wind Tunnel Testing. publisher, 1999.

[22] J. C. A. Bahaj, A. Molland and W. Batten, "Power and thrust measurements of marine current turbines under various hydrodynamic flow conditions in a cavitation tunnel and a towing tank," Renewable Energy,, vol. 32, pp. 407-426, 2007.

[23] M. Werle, "Wind turbine wall-blockage performance corrections," ournal of Propulsion and Power, vol. 26, pp. 1317-1321, 2010.

[24] H. Cao, "Aerodynamics Analysis of Small Horizontal Axis Wind Turbine Blades by Using 2D and 3D CFD Modelling," Master's thesis, University of Central Lancashire, May 2011. 
[25] F. A. R. L. A. Mcfarlane, M. El Sherif, "Aeroelastic Analysis of blade behaviour in a horizontal axis wind turbine." currently under review for the Journal of Wind Engineering Industrial Aerodynamics.

[26] T. Theodorsen, "General Theory of Aerodynamic Instability and the Mechanism of Flutter," Tech. Rep. 496, NACA, May 1934.

[27] J. Wright and J. Cooper, Introduction to Aeroelasticity and Loads. Wiley \& Sons, 2007.

[28] J. G. Leishman, Principles of Helicopter Aerodynamics. Cambridge University Press, 2006.

[29] J. W. J. Cooper, Introduction to Aeroelasticity and Loads. Wiley Sons, 2007.

[30] H. Glauert, "The Anysis of Experimental Results in Windmill Brake and Vortex Ring States of an Airscrew," tech. rep., 1926.

[31] B. B., "Develpment of Small Wind Turbines." Technical University of Denmark.

[32] K. A. Y. He, "Shape Optimization of NREL S809 Airfoil for Wind Turbine Blades Using a Multiobjective Genetic Algorithm," International Journal of Aerospace Engineering, vol. 2014, 2014.

[33] U. Tinis, "An Algorithm for the Aerodynamic Analysis of Rotating Blades Using a Weak Viscous-Inviscid Interaction Method," Master's thesis, Carleton University, 2013.

[34] W. S. Wilson R., Lissaman P., "Aerodynamic Performance of Wind Turbines," 1976.

[35] J. Parker, "ATI Mini-45-E Transducer." http://ati-ia.com/app_content/ Documents/9230-05-1315.pdf, October 2014. 


\section{Appendix A}

\section{A.1 Fluent User Defined Functions}

A sample UDF from the Theordorsen pitching and plunging motions found in Chapter 4 , trial 2 .

Harmonic pitching and plunging code

\#include "udf.h"

DEFINE_CG_MOTION(move_body, dt, vel, omega, time, dtime )

\{

Thread *t;

real ampl, plungeampl, disp, omg, omgh, count;

/* reset velocities */

NV_S (vel, =, 0.0);

NV_S (omega, =, 0.0 );

$\mathrm{t}=$ DT_THREAD $(\mathrm{dt})$;

/* motion */

plungeampl $=0.1$; 


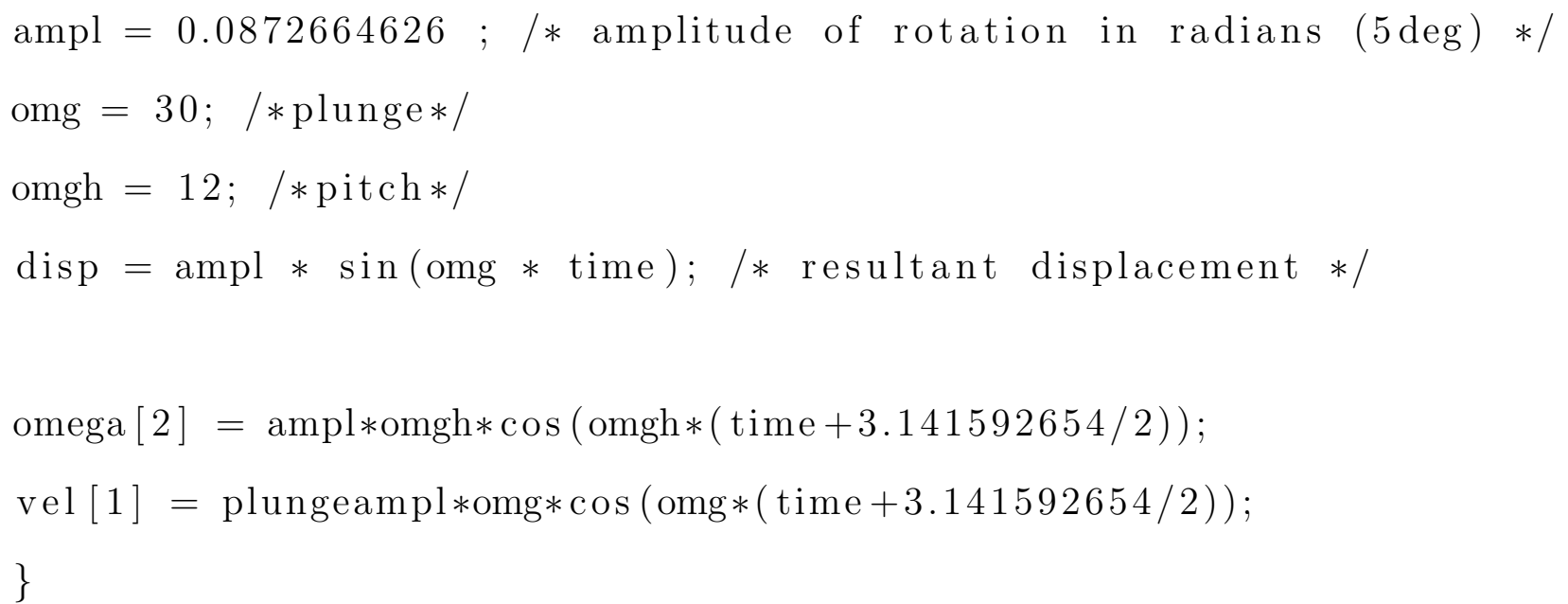




\section{Appendix B}




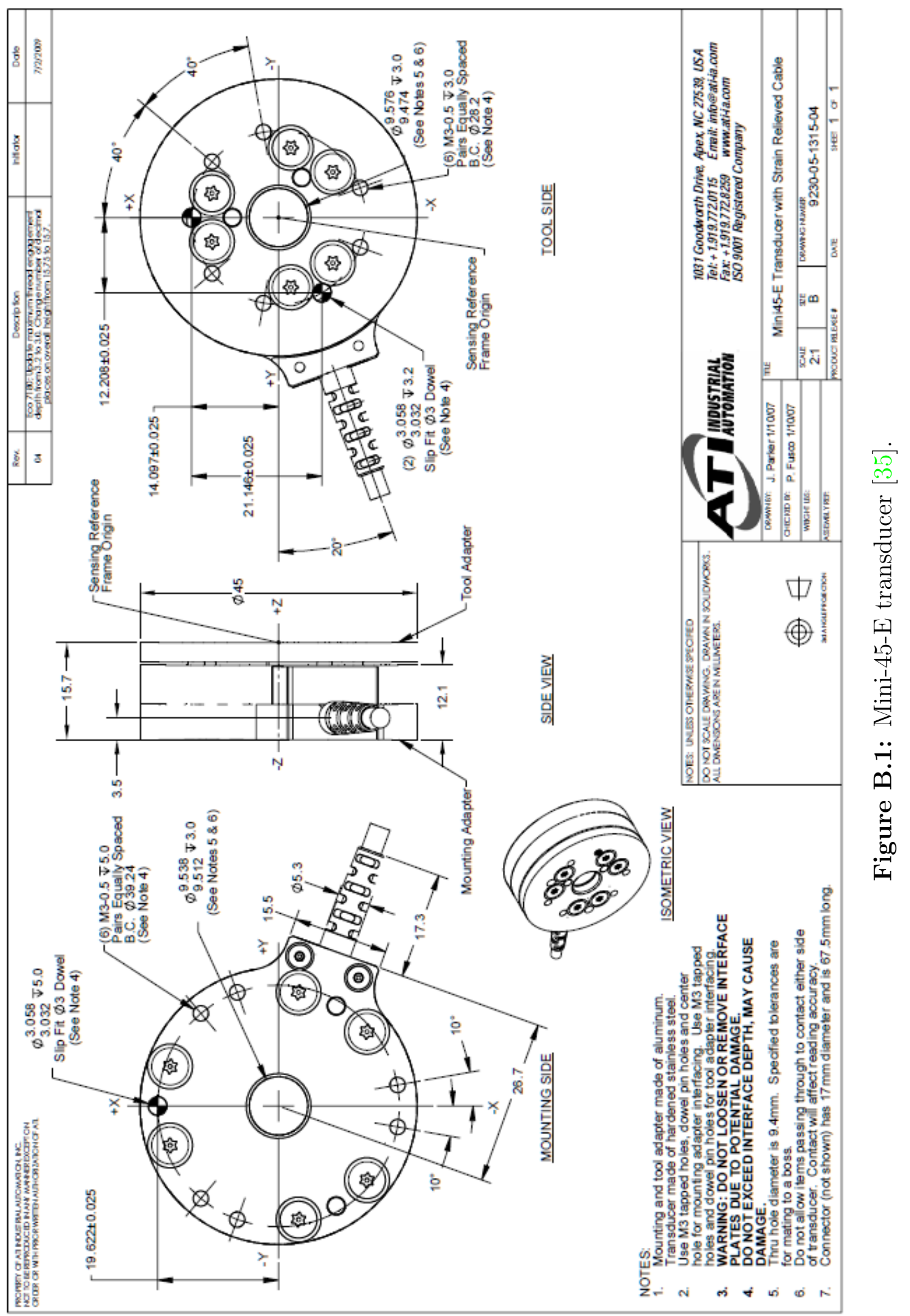




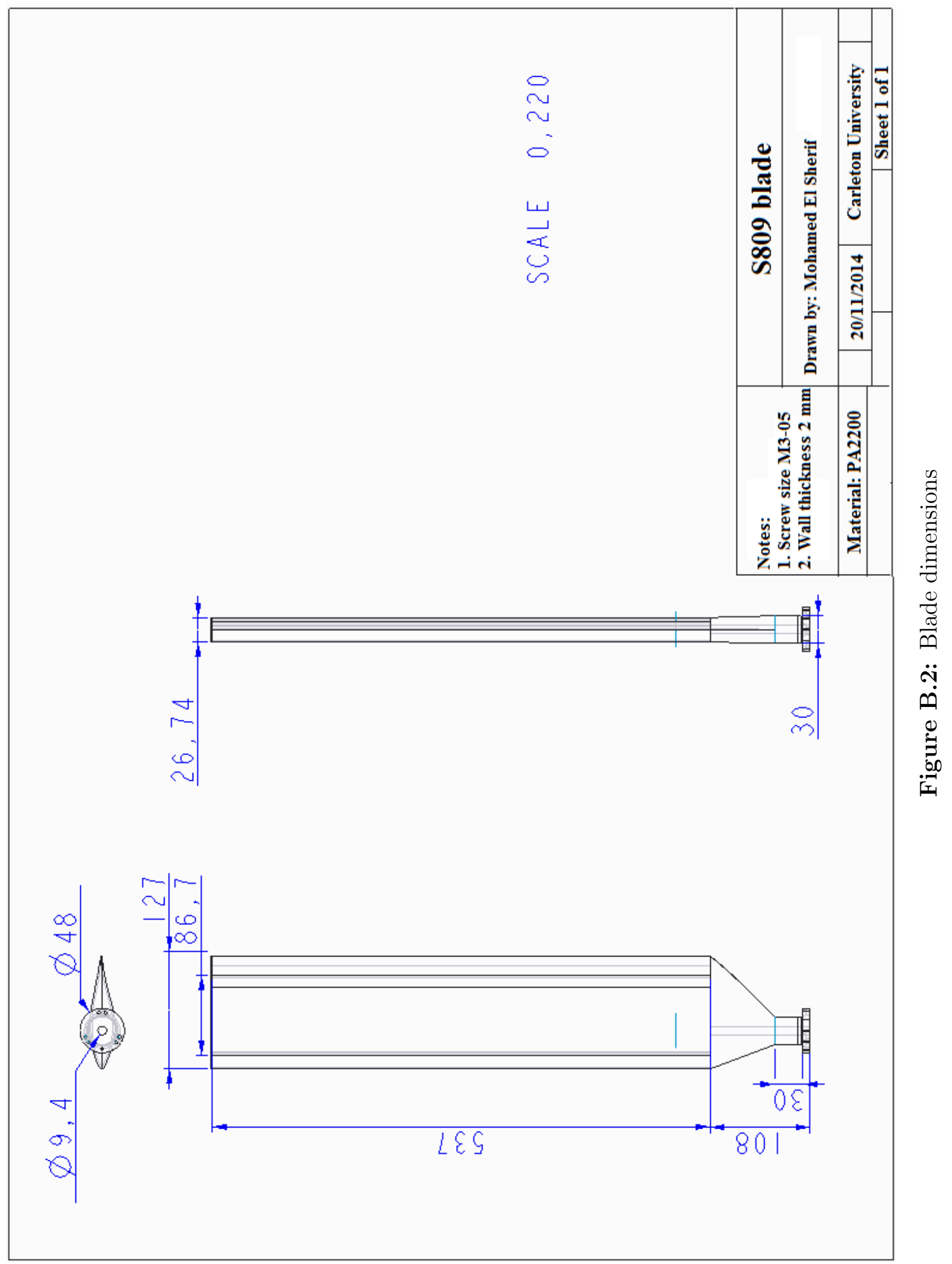




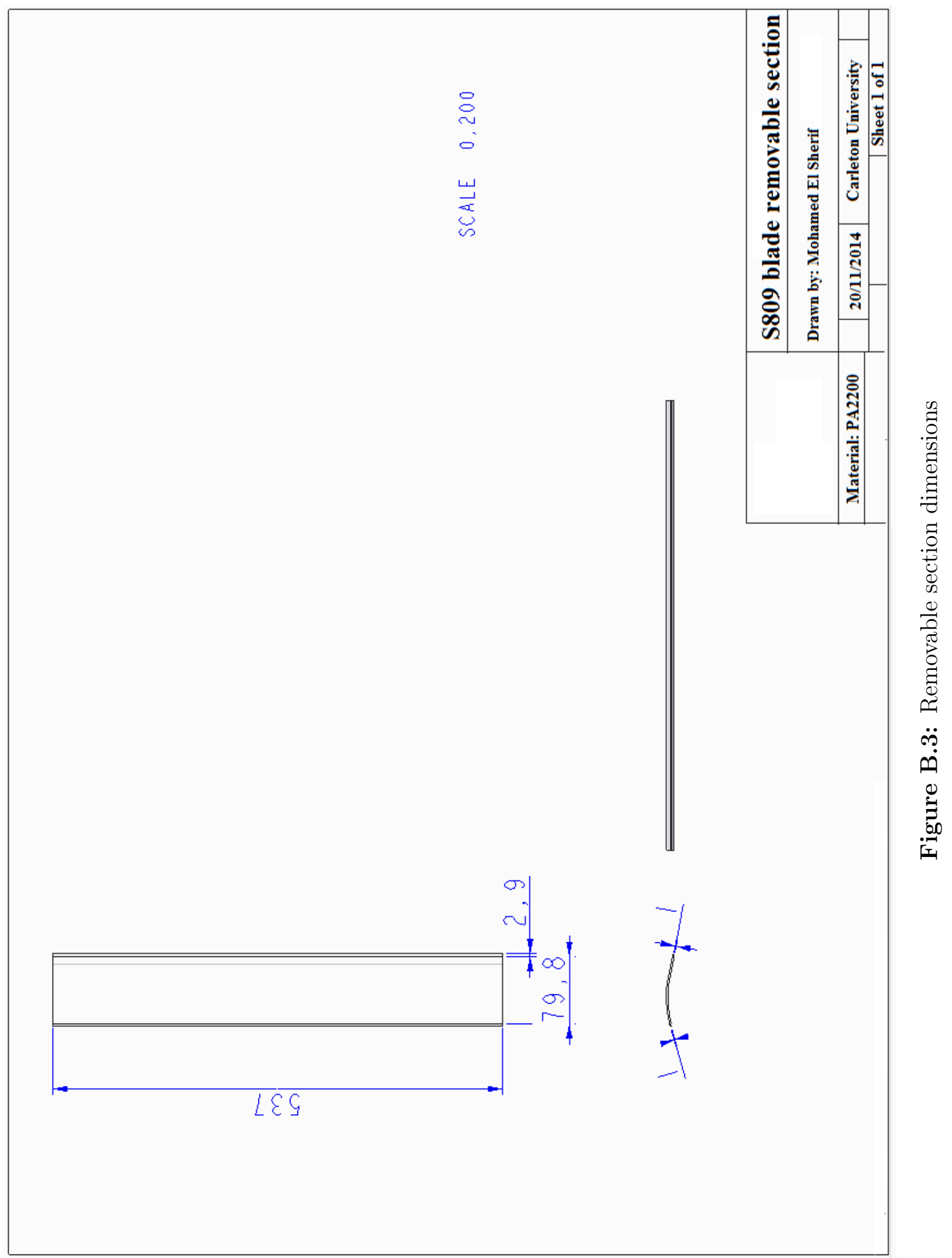




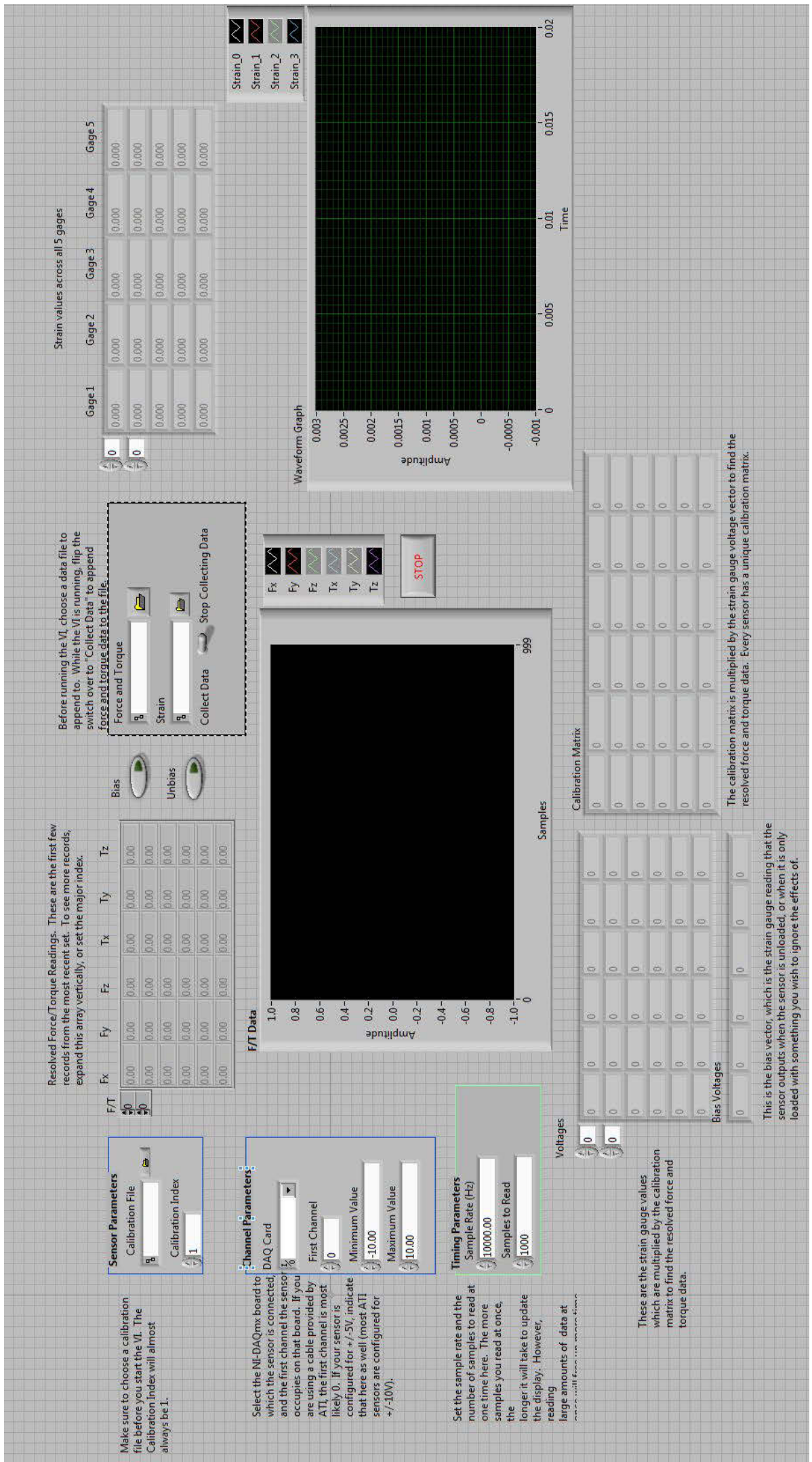

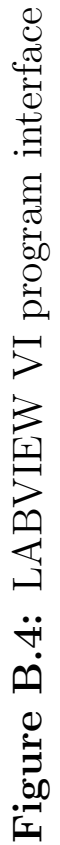




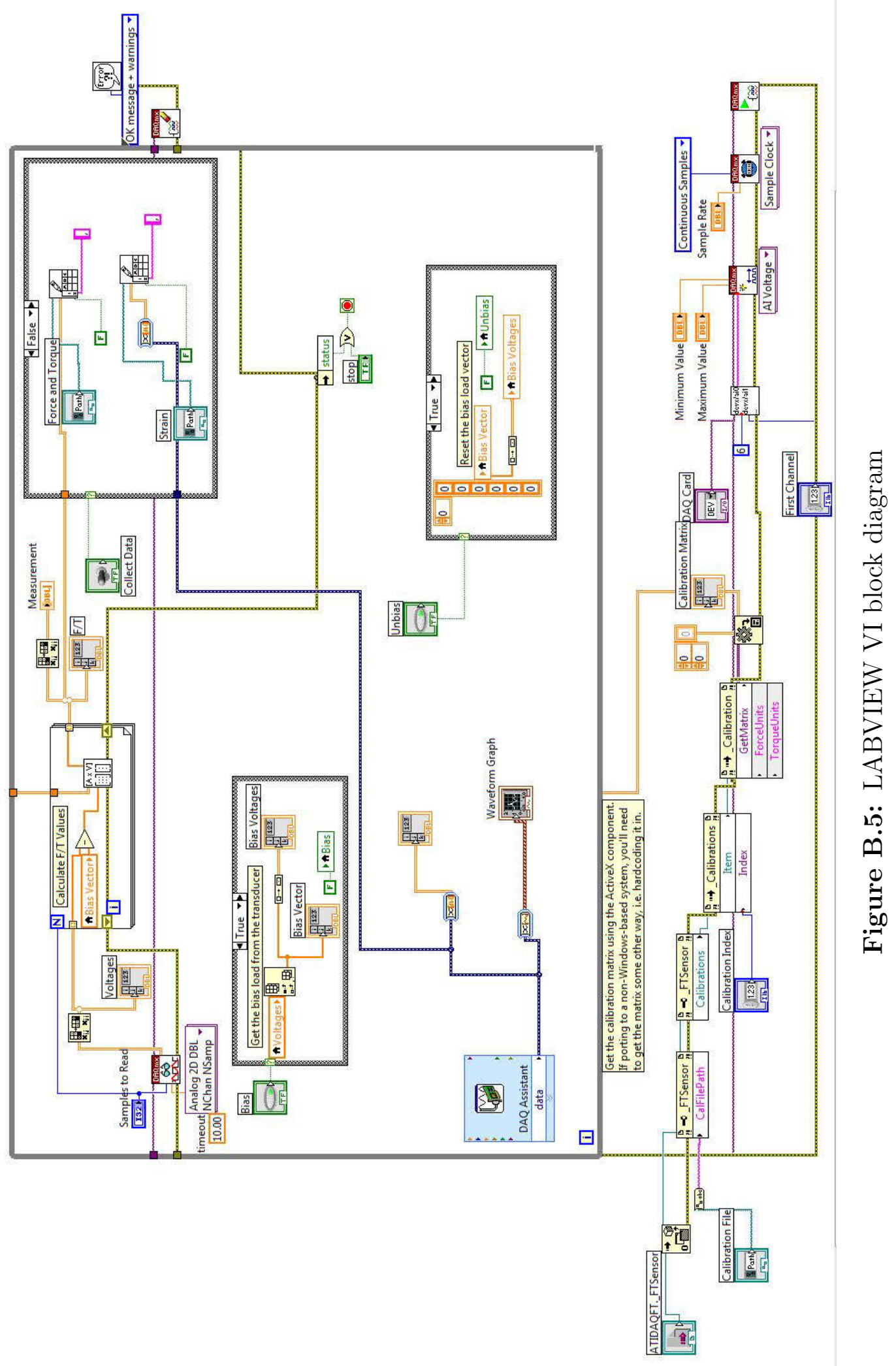

